Current practice and future perspectives in surgery for rectal prolapse 


\section{Current practice and future perspectives in surgery for rectal prolapse}

(c) H.A. Formijne Jonkers, 2014

All rights reserved. No part of this thesis may be reproduced or transmitted in any form or by any means without prior permission of the author.

ISBN: $\quad$ 978-90-365-3659-2

Lay-out: $\quad$ Roy Sanders

Cover: $\quad$ Roy Sanders

Drukwerk: Gildeprint

The printing of this thesis was financially supported by:

Universiteit Twente, Nederlandsche Vereniging voor Endoscopische Chirurgie (NVEC), Johnson \& Johnson Medical, Covidien, Takeda, Linaria B.V. 


\title{
Current practice and future perspectives in surgery for rectal prolapse
}

\author{
PROEFSCHRIFT
}

Ter verkrijging van

de graad van doctor aan de Universiteit Twente, op gezag van de rector magnificus,

prof. dr. H. Brinksma

volgens besluit van het College voor Promoties

in het openbaar te verdedigen

op donderdag 5 juni 2014 om 12.45

door

Hendrik Adriaan Formijne Jonkers

geboren op 11 juni 1984

te Enschede, Nederland. 


\section{PROMOTIECOMMISSIE}

Promotoren: Prof. Dr. I.A.M.J. Broeders Prof. Dr. W.A. Bemelman

Copromotoren: Dr. E.C.J. Consten Dr. W.A. Draaisma 


\section{CONTENT}

$\begin{array}{lll}\text { Chapter } 1 \text { General introduction. } & 9\end{array}$

Section 1. Current practice in surgery for rectal prolapse

Chapter 2 Evaluation and surgical treatment of rectal prolapse:

An international survey.

Colorectal Disease 2013 Jan;15(1):115-9.

Chapter 3 Laparoscopic ventral rectopexy for rectal prolapse

and symptomatic rectocele: an analysis of 245

consecutive patients.

Colorectal Disease 2013 Jun;15(6):695-9.

Chapter 4 Impact of rectopexy on Sexual Function:

A Cohort Analysis.

International Journal of Colorectal Disease. 2013

Nov;28(11):1579-82.

Chapter 5 Laparoscopic resection rectopexy versus laparoscopic ventral rectopexy for complete rectal prolapse.

Techniques in Coloproctology. 2014 Feb 6 (online)

Chapter 6 High-grade hemorrhoids requiring surgical treatment are common after laparoscopic ventral rectopexy.

Submitted 
Section 2. Future perspectives in surgery for rectal prolapse

Chapter 7 The optimal strategy for proximal mesh fixation

during laparoscopic ventral rectopexy for rectal

prolapse: an ex vivo study.

Surgical Endoscopy. 2012 Aug;26(8):2208-12.

Chapter 8 Robotic rectal prolapse surgery.

Chapter published in 'Robotic Surgery for the General

Surgeon'

Chapter 9 Learning curve for robotic assisted ventral rectopexy:

A cohort analysis.

Submitted

Chapter 10 Robot-Assisted Laparoscopic Ventral Rectopexy for rectal prolapse: analysis of a large cohort of patients.

Submitted

Chapter 11 General discussion

Chapter 12 Samenvatting in het Nederlands (Summary in Dutch)

Review committee

List of publications

Dankwoord (acknowledgments)

Curriculum vitae 


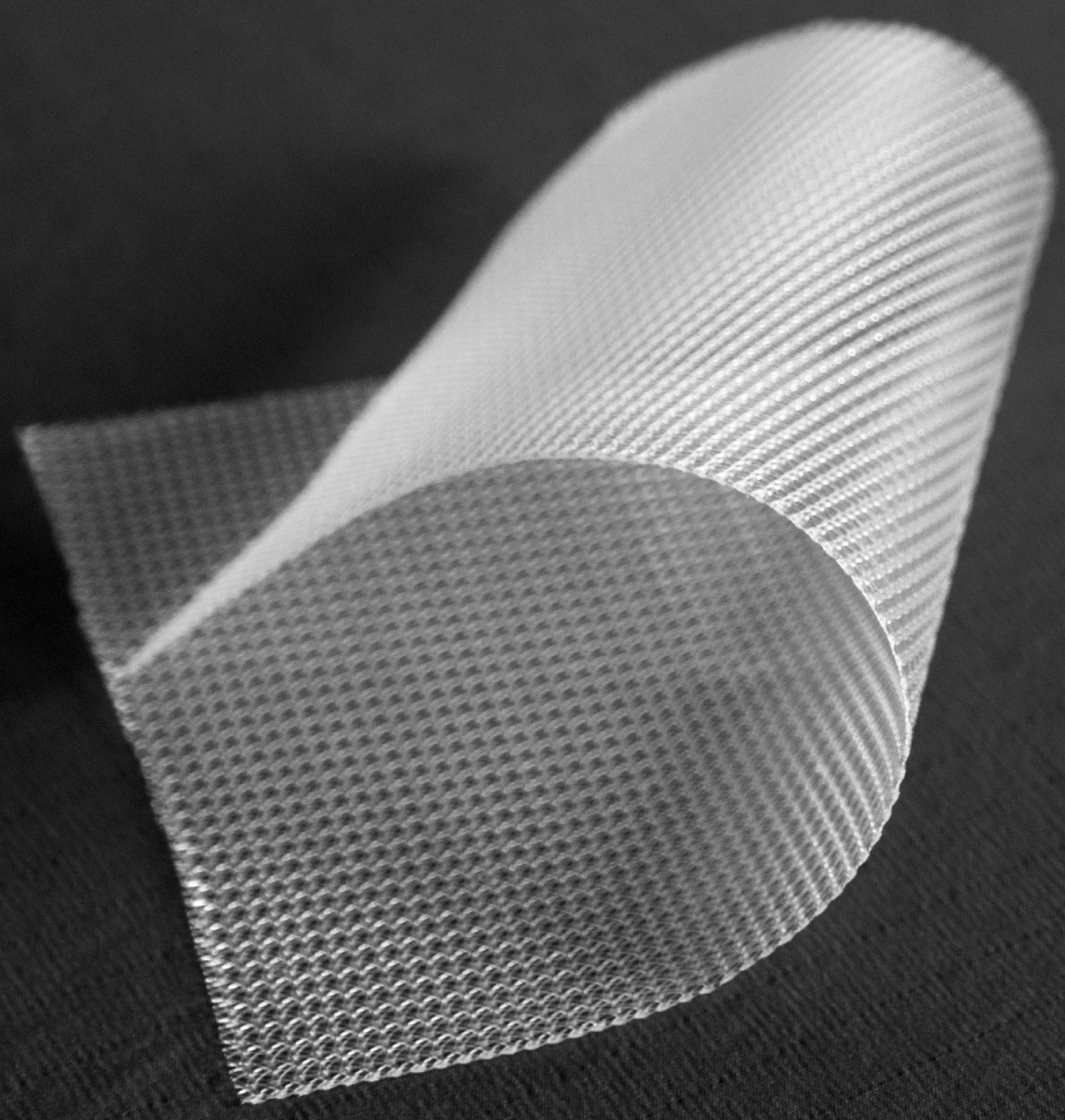




\section{Chapter 1}

General introduction 


\section{PATHOgENESIS}

The rectum is the final part of the large intestine. It stretches from the rectosigmoid junction (the end of the sigmoid colon) till the anal canal and has an average length between 10 and $12 \mathrm{~cm}$. The main function is to temporary store feces prior to defecation. Expansion of the rectal walls causes the stretch receptors within the walls to stimulate the urge to defecate. Anatomical disorder can therefore lead to functional problems of the rectum. [1;2]

Rectal Prolapse (RP) is the condition in which the upper part descents. If the rectum does not prolapse beyond the level of the anus it is called an internal $\mathrm{RP}$ or intussusception. An internal RP is the precursor to an external RP, in which the rectum protrudes through the anus. It is important to distinguish a full-thickness RP from a mucosal prolapse, in which there is protusion of only rectal or anal mucosa through the anus. [3-5]

$\mathrm{RP}$ is a relatively common disorder in the western world with an overall incidence of 420 per 100.000 people. Above the age of 65 this incidence rises up to 1000 per 100.000.[6] The majority of cases occur among women of middle and older age, mostly in combination with other prolapses of pelvic floor organs, for example descensus of the uterus and bladder.[5;7] Risk factors for RP are comparable with those of other pelvic organ prolapses and include the presence of an abnormal deep pouch of Douglas, laxity and atonic condition of the muscles of the pelvic floor and anal canal. Additionally, weakness of both internal and external sphincters, often associated with pudendal nerve neuropathy and the lack of normal fixation of the rectum, may lead to RP. [5]

\section{SYMPTOMS \& TREATMENT}

In the majority of affected patient both internal and external rectal prolapse lead to functional impairment of the rectum. Complaints include incontinence, constipation or the Obstructed Defecation Syndrome (ODS). The latter encompasses the inability to evacuate the rectum during defecation. [5;8;9]

Initial treatment should always include dietary changes and medications like bulking agents. Furthermore, specialized physical therapy of the pelvic floor, also called biofeedback therapy, can be beneficial in selected cases. The only definite treatment for RP however is surgery, especially in cases of external RP. $[10 ; 11]$

During the last century, numerous surgical treatment techniques have been described to treat RP. These techniques are used as a way to either obliterate the peritoneum of the pouch of Douglas, to narrow the anal canal, restore the pelvic floor, resect the redundant part or to suspend or fixate the rectum to the sacrum or other structures. This can be done trans-abdominally (via either laparotomy or laparoscopy) or with a perineal approach.[3;5;12] 
In general, the primary aim of these techniques is to restore anatomy and thereby improve rectal function and reduce symptoms. As recurrent prolapse is an issue in all surgical techniques, the optimal surgical procedure should lead to both functional improvement and minimize the risk of recurrence.

Perineal techniques (for example Delormes' mucosectomy and Altemeiers' perineal rectosigmoidectomy) are known to have higher recurrence rates compared to most abdominal techniques. However, they are considered less invasive and can be performed under spinal anesthesia.[13-15] Abdominal techniques such as resection rectopexy are performed under general anesthesia. As a result, abdominal techniques are reserved for fit patients while the frail and elderly should be offered a perineal or transanal technique.[14] With the introduction of laparoscopy and the continuing evolution of safer techniques in general anesthesia, abdominal approaches can be offered to a wider array of patients. Even octogenarians with a RP are nowadays safely treated using a laparoscopic approach.[16]

\section{CONTROVERSIES IN CURRENT PRACTICE.}

One of the techniques introduced in the last decade is Laparoscopic Ventral Rectopexy (LVR).[17] This mesh rectopexy technique became increasingly popular in Europe as it seems to offer a low rate of complications and good results in several cohorts of patients. [18-20] In the United States of America LVR has not gained broad acceptance yet. Laparoscopic resection rectopexy (LRR), in which a sigmoid resection is combined with a suture rectopexy, seems to remain popular. LRR is believed to have superior functional results compared to LVR as this latter might cause kinking of the "redundant" sigmoid and thereby provokes constipation de-novo after surgery.[21;22] Nonetheless, the big downside of LRR compared to LVR is the possible risk of anastomotic leakage. To date, literature is lacking regarding complications and functional outcomes after either of these procedures. Direct comparisons have not yet been published.

In 2011, the US Food and Drug Administration (FDA) released a safety warning concerning the use of meshes in pelvic floor surgery. A high incidence of pain, mesh infections and mesh erosions through the vagina with subsequent dyspareunia was found after mesh implantation for pelvic organ prolapse.[23] This warning has led to extensive reports in media on this issue and was followed by several lawsuits against doctors and the manufacturers of these meshes. Complications appear to occur predominantly after the use of transvaginal meshes implanted by gynecologists. Nevertheless, the rate of mesh related complications after LVR has not been determined yet in large studies nor has the sexual function of patients after this procedure been described. 


\section{CONTROVERSIES IN NEW TECHNOLOGIES.}

In recent years several new techniques have been introduced to improve the results of LVR. The use of a robot (Da-Vinci system) during LVR is gaining popularity as these systems are designed to offer improved visibility, maneuverability and dexterity in small spaces like the pelvis. Furthermore robots reduce ergonomic inconveniences for the surgeon. As such, using a robot is an attractive method and might lead to a better position of the mesh on the ventral aspect of the rectal wall. Ultimately, functional results of robot-assisted LVR (RALVR) might be superior to conventional LVR.[24;25] However, these advantages have to be waged against the main disadvantage of RALVR: higher costs. Furthermore, surgeons need to become proficient in RALVR and it is not clear whether the use of a robot has any influence on the rate of complications.

Another innovation is a new, specially designed screw for proximal mesh fixation during LVR. This screw was designed to offer a precise and strong mesh fixation during this procedure. Originally, both the use of several non-resorbable sutures and endostaplers were mentioned as possible techniques for proximal mesh fixation.[17] It has not been studied yet whether the use of the new screw offers similar (or perhaps even better) fixation compared to stitches, staples or tackers.

\section{OUTLINE OF THIS THESIS}

This thesis is divided into 2 sections. In section 1 (chapter 2-6) the current treatment of RP and the results of surgery are described. Section 2 (chapter 7-10) focuses on advanced technologies in the treatment of RP.

As an introduction to this thesis, chapter $\mathbf{2}$ describes current practice in the treatment of rectal prolapse throughout the world. This article discusses current controversies in practice between surgeons and countries. These controversies clarify the need for new evidence in the treatment of RP.

Chapters $\mathbf{3}$ - $\mathbf{5}$ focus on current practice in the treatment of RP. The functional results and complications of the most popular surgical technique for the treatment of RP in Europe (LVR) are described in chapter 3. In chapter 5, these results are directly compared to the most popular technique in the USA (LRR). In chapter 4 , the results regarding the post-operative sexual function of treated patients are evaluated. As closing chapter of the first section, chapter 6 highlights those patients were LVR had to be followed by surgery for large hemorrhoids. 
Chapter $\mathbf{7}$ is a technical study in which the optimal mesh fixation during LVR is studied. Chapters $\mathbf{8} \mathbf{- 1 0}$ describe the use of a robotic surgery system during LVR. Not only a review of current practice and literature is given (chapter 8), also the learning curve for robot-assisted LVR (chapter 9) and the functional results and complications of it (chapter 10) are described.

The studies presented in this thesis were guided by the following research questions:

- What is current practice in surgery for RP and are there any differences between surgeons and countries? (Chapter 2)

- What are the (functional) results of the most popular surgical techniques for the treatment of RP? (Chapters 3, 5,6 and 10)

- What is the influence of LVR on the sexual function of female patients? (Chapter 4)

- What is the incidence of mesh-related complications after LVR? (Chapter 3,4)

- Which of the possible methods of proximal mesh fixation during LVR is preferable in terms of fixation strength? (Chapter 7)

- What is the current status in the usage of a robotic surgery system in the field of rectal prolapse surgery? (Chapter 8,10$)$

- What is the learning curve for RALVR? (Chapter 9) 


\section{REFERENCE LIST}

1 Kaiser AM, Ortega AE: Anorectal anatomy. Surg Clin North Am 2002;82:1125-38, v.

2 Whitehead WE, Wald A, Diamant NE, Enck P, Pemberton JH, Rao SS: Functional disorders of the anus and rectum. Gut 1999;45 Suppl 2:II55-II59.

3 Madiba TE, Baig MK, Wexner SD: Surgical management of rectal prolapse. Arch Surg 2005;140:63-73.

4 Wijffels NA, Collinson R, Cunningham C, Lindsey I: What is the natural history of internal rectal prolapse? Colorectal Dis 2010;12:822-830.

5 Wu JS: Rectal prolapse: a historical perspective. Curr Probl Surg 2009;46:602-716.

6 Kairaluoma MV, Kellokumpu IH: Epidemiologic aspects of complete rectal prolapse. Scand J Surg 2005;94:207-210.

7 Elneil S: Complex pelvic floor failure and associated problems. Best Pract Res Clin Gastroenterol 2009;23:555-573.

8 Altomare DF, Spazzafumo L, Rinaldi M, Dodi G, Ghiselli R, Piloni V: Set-up and statistical validation of a new scoring system for obstructed defaecation syndrome. Colorectal Dis 2008;10:84-88.

9 Wijffels NA, Jones OM, Cunningham C, Bemelman WA, Lindsey I: What are the symptoms of internal rectal prolapse? Colorectal Dis 2013;15:368-373.

10 Braekken IH, Majida M, Engh ME, Bo K: Can pelvic floor muscle training reverse pelvic organ prolapse and reduce prolapse symptoms? An assessor-blinded, randomized, controlled trial. Am J Obstet Gynecol 2010;203:170-177.

11 Hwang YH, Person B, Choi JS, Nam YS, Singh JJ, Weiss EG, Nogueras JJ, Wexner SD: Biofeedback therapy for rectal intussusception. Tech Coloproctol 2006;10:11-15.

12 Kuijpers HC: Treatment of complete rectal prolapse: to narrow, to wrap, to suspend, to fix, to encircle, to plicate or to resect? World J Surg 1992;16:826-830.

13 Milito G, Cadeddu F, Selvaggio I, Grande M: The Delorme repair for full-thickness rectal prolapse: a retrospective review. Am J Surg 2010;199:581-582.

14 Riansuwan W, Hull TL, Bast J, Hammel JP, Church JM: Comparison of perineal operations with abdominal operations for full-thickness rectal prolapse. World J Surg 2010;34:1116-1122.

15 Sehmer D, Marti L, Wolff K, Hetzer FH: Midterm results after perineal stapled prolapse resection for external rectal prolapse. Dis Colon Rectum 2013;56:91-96.

16 Wijffels N, Cunningham C, Dixon A, Greenslade G, Lindsey I: Laparoscopic ventral rectopexy for external rectal prolapse is safe and effective in the elderly. Does this make perineal procedures obsolete? Colorectal Dis 2011;13:561-566.

17 D’hoore A, Penninckx F: Laparoscopic ventral recto(colpo)pexy for rectal prolapse: surgical technique and outcome for 109 patients. Surg Endosc 2006;20:1919-1923. 
18 Boons P, Collinson R, Cunningham C, Lindsey I: Laparoscopic ventral rectopexy for external rectal prolapse improves constipation and avoids de novo constipation. Colorectal Dis 2010;12:526532.

19 D’hoore A, Cadoni R, Penninckx F: Long-term outcome of laparoscopic ventral rectopexy for total rectal prolapse. Br J Surg 2004;91:1500-1505.

20 van den Esschert JW, van Geloven AA, Vermulst N, Groenedijk AG, de Wit LT, Gerhards MF: Laparoscopic ventral rectopexy for obstructed defecation syndrome. Surg Endosc 2008;22:27282732.

21 Laubert T, Kleemann M, Schorcht A, Czymek R, Jungbluth T, Bader FG, Bruch HP, Roblick UJ: Laparoscopic resection rectopexy for rectal prolapse: a single-center study during 16 years. Surg Endosc 2010;24:2401-2406.

22 von PM, Ashari LH, Lumley JW, Stevenson AR, Stitz RW: Functional results of laparoscopic resection rectopexy for symptomatic rectal intussusception. Dis Colon Rectum 2007;50:50-55.

23 Rogers RG: To mesh or not to mesh: current debates in prolapse repair fueled by the U.S. Food and Drug Administration Safety Notification. Obstet Gynecol 2011;118:771-773.

24 Mantoo S, Podevin J, Regenet N, Rigaud J, Lehur PA, Meurette G: Is robotic-assisted ventral mesh rectopexy superior to laparoscopic ventral mesh rectopexy in the management of obstructed defaecation? Colorectal Dis 2013;15:e469-e475.

25 Perrenot C, Germain A, Scherrer ML, Ayav A, Brunaud L, Bresler L: Long-term outcomes of robotassisted laparoscopic rectopexy for rectal prolapse. Dis Colon Rectum 2013;56:909-914. 


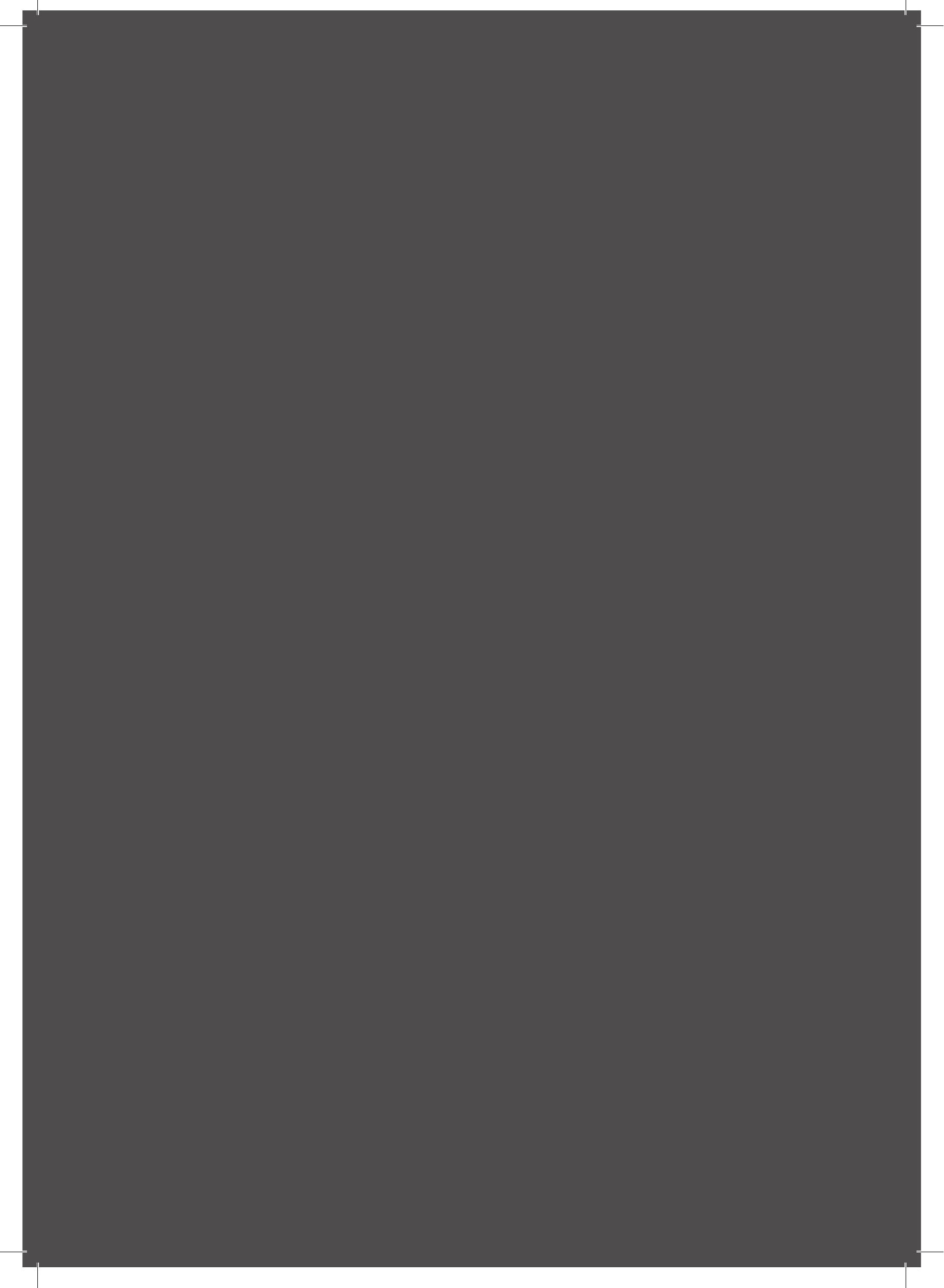




\section{SECTION 1}

Current practice in surgery for rectal prolapse 


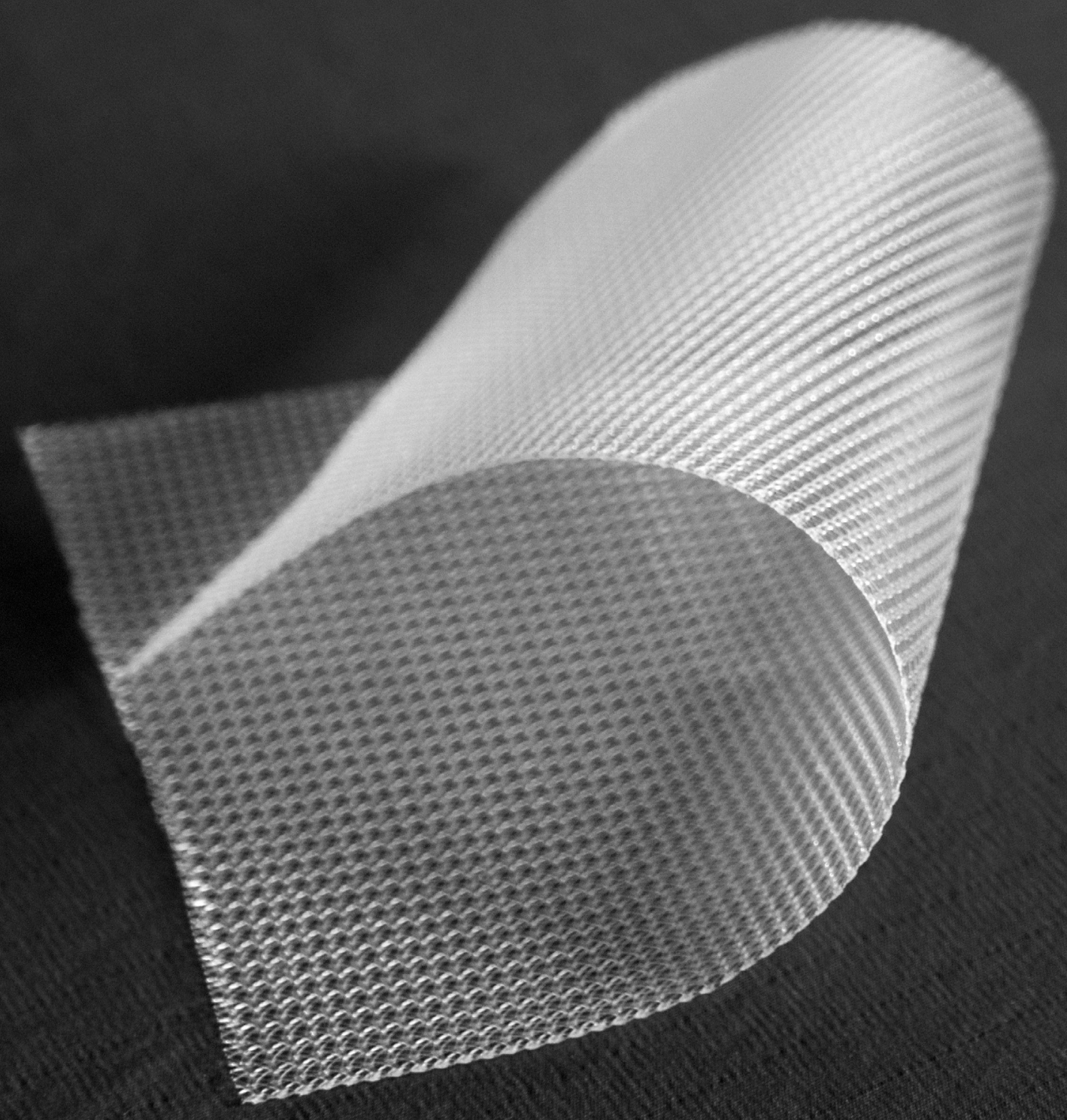




\section{Chapter 2}

\section{Evaluation and surgical treatment of rectal prolapse: \\ an international survey}

H.A. Formijne Jonkers

W.A. Draaisma

S.D. Wexner

I.A.M.J. Broeders

W.A. Bemelman

I. Lindsey

E.C.J. Consten 


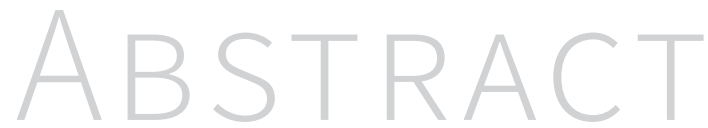

\section{Alm}

Validated guidelines for the surgical and non-surgical treatment of rectal prolapse (RP) do not exist. The aim of this international questionnaire survey was to provide an overview of the evaluation, follow-up and treatment of patients with an internal or external RP.

\section{METHOD}

A questionnaire with 36 questions in English about the evaluation, treatment and follow-up of patients with a RP was distributed amongst surgeons attending the congresses of the European Association for Endoscopic Surgery (EAES) and of the European Society of Coloproctology (ESCP) in 2010. The survey was subsequently sent to all the members of the American Society of Colon and Rectal Surgeons (ASCRS) and the ESCP by e-mail.

\section{RESULTS}

Three-hundred-ninety one surgeons originating from 50 different countries completed the questionnaire. Evaluation, surgical treatment and follow-up of patients with a RP considerably differed. For healthy patients with an external RP, laparoscopic ventral rectopexy (LVR) was the most popular treatment in Europe, whereas laparoscopic resection rectopexy (LRR) was favored in North America. There was consensus only on frail and/or elderly patients with an external prolapse, with a preference for transanal techniques. After failure of conservative therapy, internal RP was mostly treated with LRR in North America. In Europe, LVR and stapled transanal rectal resection (STARR) seemed popular techniques for these patients.

\section{CONCLUSION}

The treatment of patients with a RP differs between surgeons, countries and regions. Guidelines for the treatment of patients with this disorder are lacking. Therefore, prospective comparative studies are warranted that may result in applicable guidelines worldwide. 


\section{INTRODUCTION}

Rectal prolapse (RP) is due to full-thickness intussusception of the upper rectum, which may be internal or extrude externally. Both forms may be associated with obstructed defecation and/or fecal incontinence [1,2].

Different treatment strategies have been described including conservative treatment by relief of constipation and incontinence with dietary manipulation, laxatives, enemas and biofeedback [1,3-5]. Surgery is indicated in patients with an external RP or when conservative treatment fails to result in sufficient reduction of symptoms. Surgery includes abdominal and perineal procedures $[1,6-8]$, but prospective studies comparing different approaches and techniques are lacking. There is therefore no consensus on the best treatment for this condition, nor are there any generally accepted guidelines.

A prospective international survey was carried out to obtain a global picture of the variation in assessment and management of RP.

\section{MATERIALS \& METHODS}

A prospective international cross-sectional questionnaire survey was carried out of the treatment of RP by colorectal and general surgeons working on different continents. An English language questionnaire consisting of 36 questions was designed to obtain details on patient demographics, type of hospital and the personal practice of the surgeon in treating pelvic floor disorders. The respondents were required to state their protocol for the evaluation and treatment of patients with suspected RP. They were asked to suggest a treatment technique in eight scenarios of common and less common cases of internal and external prolapse. The surgeons' opinion on currently available literature on RP treatment was also sought.

The questionnaire was distributed during the 18th Annual Congress of the European Association for Endoscopic Surgery (EAES), held between 16 and 19 June 2010 in Geneva, Switzerland, after approval by the EAES Research Committee. It was also given to surgeons attending the 5th annual meeting of the European Society of Coloproctology (ESCP), which took place from 22 to 25 September 2010 in Sorrento, Italy, with the approval of the Scientific Committee of the ESCP. In collaboration with the American Society of Colon and Rectal Surgeons (ASCRS), an online version of the questionnaire was developed. This was sent by e-mail to all the active members as a dedicated survey. In addition, the ESCP also sent out the online version of the questionnaire to all their members. 


\section{Statistical analysis}

A database was created with SPSS version 17.0 (SPSS, Chicago, Illinois, USA). Descriptive statistics were used for statistical analysis. Continuous variables were presented as means and percentages. Categorical variables were described as counts and/or percentages

\section{RESULTS}

\section{Respondent characteristics}

A total of 391 surgeons from 50 different countries completed the survey. During the EAES and ESCP congresses, 50 questionnaires were completed. The others were returned after the mailing to all active members of the ASCRS and ESCP; there were no duplicated entries.

The geographic distribution of respondents is shown in Table 1. Ninety per cent had undergone specific colorectal training and, in addition, $73 \%$ were certified by the ASCRS or the ESCP. Most respondents (53\%) had more than 15 years'

Table 1. Geographic distribution of respondents. Countries with eight or more respondents are specified. Countries with fewer respondents are combined per region in the 'other' groups.

\begin{tabular}{|c|c|c|}
\hline Continent / Region & Country & Number of respondents \\
\hline \multicolumn{3}{|c|}{ North America (Total: 194) } \\
\hline & United States of America & 185 \\
\hline & Canada & 9 \\
\hline \multicolumn{3}{|l|}{ Europe (Total: 129) } \\
\hline & United Kingdom & 21 \\
\hline & Netherlands & 15 \\
\hline & Italy & 15 \\
\hline & Germany & 8 \\
\hline & Spain & 8 \\
\hline & Other & 62 \\
\hline \multicolumn{3}{|l|}{ Asia (Total: 27) } \\
\hline & South Korea & 8 \\
\hline & Other & 19 \\
\hline \multicolumn{3}{|c|}{ Middle and South America (Total: 18) } \\
\hline & Other & 18 \\
\hline \multicolumn{3}{|l|}{ Australia (Total: 8) } \\
\hline & Other & 8 \\
\hline Other / Not stated & & 15 \\
\hline Total & 391 & \\
\hline
\end{tabular}


experience in RP surgery. Most (47\%) practiced in a general teaching hospital or in a university department (30\%) and $9 \%$ worked in a specialized pelvic floor center.

RP surgery was performed in a multidisciplinary team setting by $56 \%$ of respondents. In addition to the surgeon, the team consisted of gynecologist (81\%), radiologist (72\%), urologist (59\%) and pelvic floor physical therapist (57\%). More rarely other specialists including gastroenterologist (3\%), neurologist (1\%) and specialist nurse $(1 \%)$ were involved. None of the respondents indicated the existence of a local protocol for the treatment of RP.

\section{Evaluation of patients with RP}

An evacuation proctogram was the most commonly performed radiological examination, being routine for $72 \%$ of respondents. A dynamic pelvic floor MRI scan was a standard preoperative investigation for $23 \%$ of surgeons. An endoanal ultrasound was routinely performed by $42 \%$ of surgeons and manometry was regarded as essential by $48 \%$ of surgeons. Only $14 \%$ routinely performed a colonoscopy or sigmoidoscopy.

\section{Treatment of RP}

For internal and external RP, 7\% of surgeons started treatment with biofeedback, regardless of the findings of radiological imaging. An additional $46 \%$ of respondents advised this treatment only if it was likely to be useful. Almost half of the surgeons (53\%) never used biofeedback.

Eight typical case scenarios are shown in Table 2. For external RP, abdominal techniques were more frequently used for all groups of patients, except for those who were frail or very elderly for whom a perineal approach was favored by $86 \%$ of surgeons. After failure of conservative treatment, internal RP was mostly treated by a perineal technique, including frail and elderly patients. Seven per cent of surgeons would never operate on a healthy patient with an internal RP. Between $34 \%$ and $39 \%$ of surgeons performed surgery by per anal approach for the different groups of healthy patients with an internal RP. Abdominal procedures were preferred for these groups of patients by the majority of surgeons, however.

\section{International differences}

Several international differences in the treatment of RP were detected. In North America, laparoscopic resection rectopexy (LRR) was favored for the treatment of external RP in healthy women in the age ranges $18-50$ and $50-80$ years by $44 \%$ and $34 \%$ of surgeons, respectively. Thirty-nine per cent of North American respondents preferred LRR for the treatment of healthy males with an external RP. In Europe, laparoscopic ventral rectopexy (LVR) was the treatment of choice 
Table 2. Overview of favored treatment techniques in 8 typical cases of patients with an internal or external rectal prolapse. Concerns surgical therapy after failure of conservative therapy. The "(Other) open approach" and "Other Technique" groups concern not specified techniques and other techniques (with a frequency of $10 \%$ or less each.

Preferred treatment method

\author{
Preferred treatment method
}

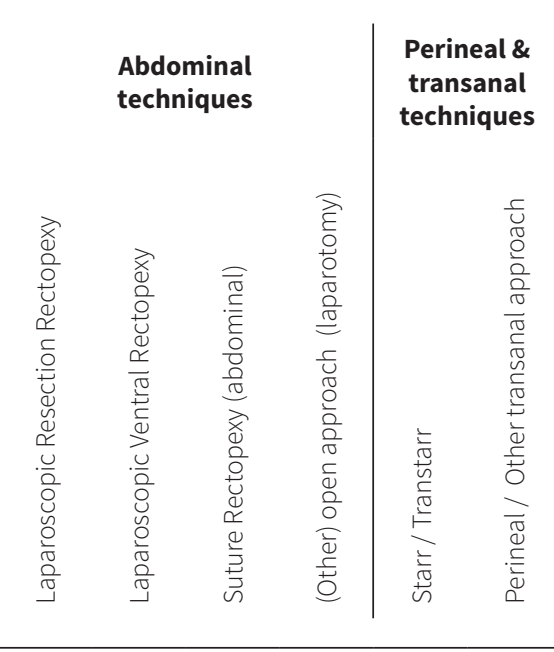

\section{What surgical treatment do you prefer for the treatment of}

external rectal prolapse in healthy women between

18 and 50 years of age?

external rectal prolapse in healthy women between

50 and 80 years of age?

external rectal prolapse in healthy men?

for external rectal prolapse in frail and / or very elderly patients?

internal rectal prolapse in healthy women between 18 and 50 years of age?

internal rectal prolapse in healthy women between 50 and 80 years of age?

internal rectal prolapse in healthy men?

internal rectal prolapse in frail and / or very elderly patients?

\begin{tabular}{|c|c|c|c|c|c|c|c|}
\hline $39.8 \%$ & $22.8 \%$ & $2.2 \%$ & $20.6 \%$ & \multicolumn{2}{|c|}{$9.9 \%$} & $0 \%$ & $4.7 \%$ \\
\hline $29.6 \%$ & $19.4 \%$ & $1.1 \%$ & $15.4 \%$ & & $2 \%$ & $0 \%$ & $1.3 \%$ \\
\hline $41.4 \%$ & $19.1 \%$ & $1.3 \%$ & $16.6 \%$ & & .1 & $0 \%$ & $1.5 \%$ \\
\hline $2.3 \%$ & $6.6 \%$ & $0.7 \%$ & $3.9 \%$ & & $9 \%$ & $0 \%$ & $0.6 \%$ \\
\hline $20.8 \%$ & $20.1 \%$ & $0 \%$ & $17.4 \%$ & $22.5 \%$ & $11.3 \%$ & $6.8 \%$ & $1.1 \%$ \\
\hline $18.4 \%$ & $18.8 \%$ & $1.4 \%$ & $15.2 \%$ & $23.8 \%$ & $14.1 \%$ & $6.9 \%$ & $1.4 \%$ \\
\hline $21.8 \%$ & $15.9 \%$ & $0.4 \%$ & $15.1 \%$ & $23.8 \%$ & $15.1 \%$ & $8.3 \%$ & $1.6 \%$ \\
\hline $7.1 \%$ & $7.9 \%$ & $0 \%$ & $3.7 \%$ & $27.4 \%$ & $40.2 \%$ & $12 \%$ & $1.7 \%$ \\
\hline
\end{tabular}


for patients with an external prolapse and $43 \%$ of respondents would perform an LVR for healthy women between 18 and 50 years. For healthy women between 50 and 80 years and for the treatment of men with an external RP, this technique was preferred by $42 \%$ and $35 \%$ of the respondents. For internal prolapse, treatment also varied between these two geographical regions. Healthy women between the ages of 18 and 50 years experiencing failure of conservative treatment were in some cases treated by LRR in North America, while in Europe stapled transanal rectal resection (STARR, 26\%) and LVR (34\%) were common. Almost identical results and percentages were obtained for women between the ages of 50 and 80 years with an internal RP.

\section{Follow-up}

No consensus was found on the protocol for follow-up. Some surgeons did not perform follow-up at all, some performed follow-up only once after 2 months while others performed annual visits for 5 years after surgery. In $24.6 \%$ of participants a locally developed questionnaire was used during follow-up, and $11.8 \%$ of surgeons performed radiological imaging regularly during follow-up. Others performed it only if clinically indicated.

\section{Current literature and future research}

The vast majority of surgeons graded the quality of the current literature on RP as moderate (65\%) or poor (28\%). Additionally, $87 \%$ of respondents stated that future prospective comparative research is warranted for the treatment of patients with RP.

\section{Discussion}

The outcome of this survey provides a comprehensive global insight into the evaluation, treatment and follow-up of patients with RP. With almost 400 returned questionnaires, the results of this survey illustrate a representative overview for the practice of colorectal surgeons in the field of RP surgery. The lack of national and international guidelines is noticeably reflected in the results. No uniformity could be identified in the evaluation, treatment and follow-up of patients with internal or external RP. Consequently, major differences were found between treatments in different international regions. The aim of this pragmatic survey study was to provide an overview of treatment modalities in different countries, rather than to determine whether type of surgery or training might influence outcome.

The treatment choices for patients with RP have been discussed for many years. Numerous conservative treatments and more than 300 surgical procedures have been described [1,6-8]. In order to gain insight into the internationally favored treatment strategies for both internal and external RP, respondents 
were asked to suggest a treatment technique in eight scenarios of common and less common cases of both internal and external prolapse. In agreement with current literature, conformity between physicians was detected in only one subgroup of patients. Perineal techniques were preferred for frail and elderly patients with an external prolapse $[7,9]$. The results for other typical groups of patients, however, showed major differences in the treatment used between centers, countries and regions. In general, abdominal operations were the most popular for both internal and external RP in healthy patients of either gender. This preference is probably caused by numerous studies describing higher recurrence rates after perineal compared with abdominal procedures [7,9].

LVR was the technique of choice for many surgeons for external RP in Europe and was also frequently used for internal RP. Several studies have shown good results following this procedure [10-12]. In the USA, LRR (laparoscopic Frykman-Goldberg procedure) is still regarded as the gold standard for external RP [13]. Unfortunately, no comparative studies of the two techniques have been made. Owing to the small numbers of responders from other continents, it was not possible to determine the most frequently applied treatments outside Europe and the USA.

The study also demonstrated lack of multidisciplinary treatment of patients with RP in almost half the centers. RP is often associated with the descent of other pelvic organs [14]. The choice of radiological examination for the evaluation of patients with a suspected RP is also a matter of debate. This survey demonstrates that evacuation proctography is the favored investigation by the vast majority of surgeons. Dynamic MRI is a modern alternative, offering a view of all the pelvic floor organs and supporting musculature. Unfortunately, there has been no comparison between the accuracy of these different modalities, except for a small postoperative study [15]. In patients with an internal and external RP and associated fecal incontinence, sphincter function measured by manometry has been shown to correlate with the grade of prolapse [16]. Nevertheless, more than half of participating surgeons did not perform manometry, possibly because the results would not influence the choice of therapy.

Prospective comparative studies on the surgical treatment of RP are needed as stated by Madoff and Mellgren in 1999 [17]. Although several new techniques have been described since that time, only three prospective studies have been initiated including the DeloRes Trial comparing LRR and Delorme's operation [18], the LaProS Study focusing on LVR and LRR [19] and the Prosper Trial comparing rectopexy (with or without resection) to perineal surgery (Altemeier's and Delorme's procedures) [20]. The results of these studies might lead to new understanding of the optimal operation for RP. 
In summary, the results of the survey demonstrate major differences worldwide in the evaluation and treatment of internal and external RP. This could be due to the lack of comparative studies and guidelines. The results of the few ongoing prospective studies are awaited.

\section{ACKNOWLEDGEMENTS}

We gratefully would like to thank the ASCRS and ESCP for their help and support of this project, in special for online dissemination of the questionnaire among all active members of both these societies. 


\section{REFERENCES}

1 Wu JS. Rectal prolapse: a historical perspective. Curr Probl Surg2009; 46: 602-716.

2 Wijfels NA, Collinson R, Cunningham C, Lindsey I. What is the natural history of internal rectal prolapse?Colorectal Dis2010; 12: 822-30.

3 Hwang YH, Person B, Choi JS et al. Biofeedback therapy for rectal intussusception. Tech Coloproctol2006; 10: 11-5.

4 Kraemer M, Ho YH, Tan W. Effectiveness of anorectal biofeedback therapy for fecal incontinence: medium-term results. Tech Coloproctol2001; 5: 125-9.

5 Wang J, Luo MH, Qi QH, Dong ZL. Prospective study of biofeedback retraining in patients with chronic idiopathic functional constipation. World J Gastroenterol2003; 9: 2109-13.

6 Kuijpers HC. Treatment of complete rectal prolapse: to narrow, to wrap, to suspend, to fix, to encircle, to plicate or to resect?World J Surg1992; 16: 826-30.

7 Madiba TE, Baig MK, Wexner SD. Surgical management of rectal prolapse. Arch Surg2005; 140: 63-73.

8 Shin EJ. Surgical treatment of rectal prolapse. J Korean Soc Coloproctol2011; 27: 5-12.

9 Tou S, Brown SR, Malik Al, Nelson RL. Surgery for complete rectal prolapse in adults. Cochrane Database Syst Rev2008; 4: CD001758.

10 D'Hoore A, Cadoni R, Penninckx F. Long-term outcome of laparoscopic ventral rectopexy for total rectal prolapse. Br J Surg2004; 91: 1500-5.

11 D’Hoore A, Penninckx F. Laparoscopic ventral recto(colpo)pexy for rectal prolapse: surgical technique and outcome for 109 patients. Surg Endosc2006; 20: 1919-23.

12 Collinson R, Wijffels N, Cunningham C, Lindsey I. Laparoscopic ventral rectopexy for internal rectal prolapse: short-term functional results. Colorectal Dis2010; 12: 97-104.

13 Laubert T, Kleemann M, Schorcht A et al. Laparoscopic resection rectopexy for rectal prolapse: a single-center study during 16 years. Surg Endosc2010; 24: 2401-6.

14 Elneil S. Complex pelvic floor failure and associated problems. Best Pract Res Clin Gastroenterol2009; 23: 555-73.

15 Otto SD, Oesterheld A, Ritz JP et al. Rectal anatomy after rectopexy: cinedefecography versus MRdefecography. J Surg Res2011; 165: 52-8.

16 Harmston C, Jones OM, Cunningham C, Lindsey I. The relationship between internal rectal prolapse and internal anal sphincter function. Colorectal Dis2011; 13: 791-5.

17 Madoff RD, Mellgren A. One hundred years of rectal prolapse surgery. Dis Colon Rectum1999; 42: 441-50.

18 DeloRes Trial. http://www.delores-trial.eu (accessed 1 March 2012). 
19 LaProS Study. http://www.trialregister.nl/trialreg/admin/rctview.asp?TC=2743 (accessed 1 March 2012).

20 Prosper Trial. http://www.prosper.bham.ac.uk/ (accessed 1 March 2012) 


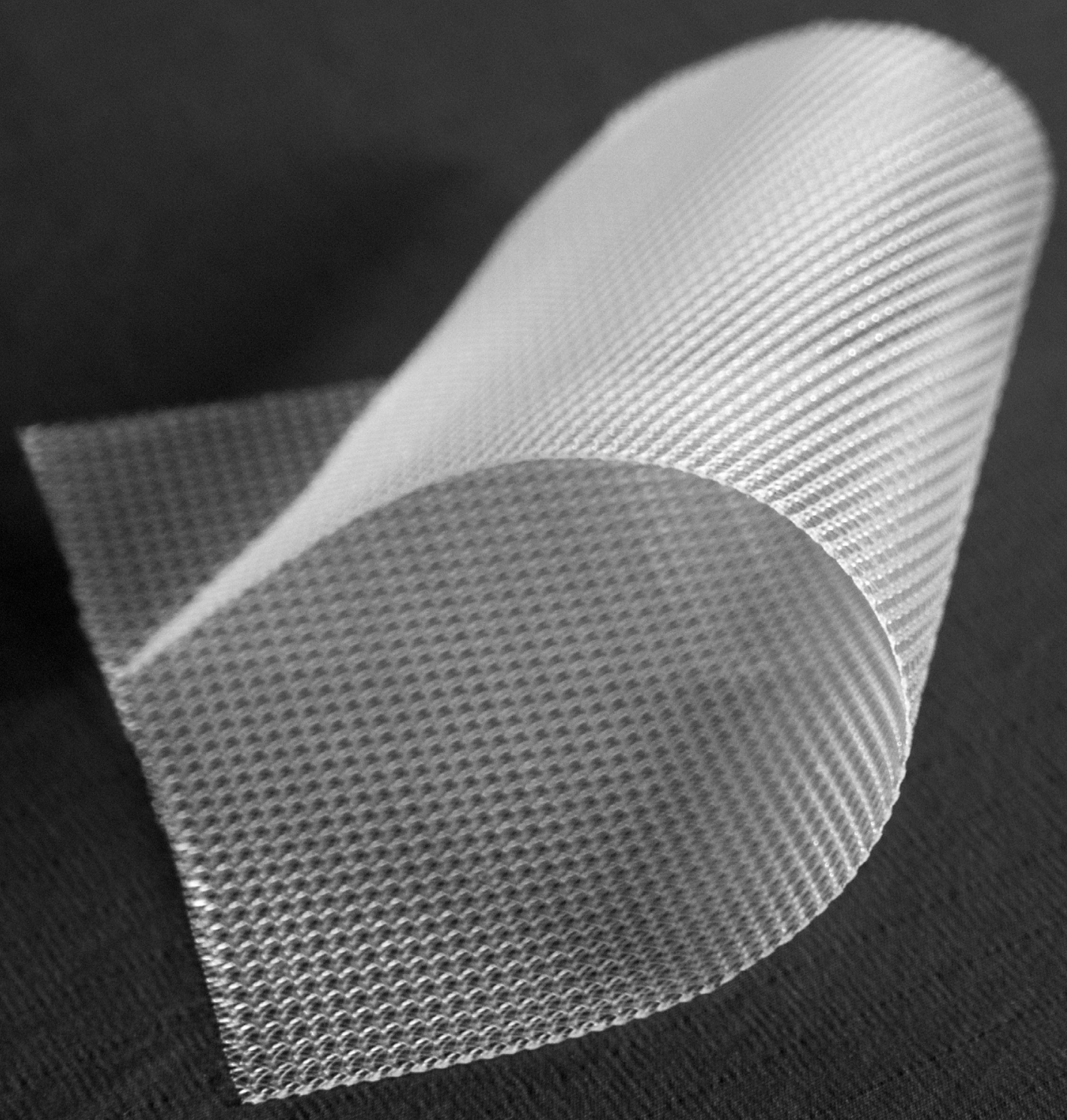




\section{Chapter 3}

Laparoscopic ventral rectopexy for rectal prolapse and symptomatic rectocele: an analysis of 245 consecutive patients 
Aim

This retrospective study aimed to determine functional results of laparoscopic ventral rectopexy (LVR) for rectal prolapse (RP) and symptomatic rectoceles in a large cohort of patients.

\section{Method}

All patients treated between 2004 and 2011 were identified. Relevant patient characteristics were gathered. A questionnaire concerning disease-related symptoms as well as the Cleveland Clinic Incontinence Score (CCIS) and Cleveland Clinic Constipation Score (CCCS) was sent to all patients.

\section{Results}

A total of 245 patients underwent operation. Twelve patients (5\%) died during follow-up and were excluded. The remaining patients (224 women, nine men) were sent a questionnaire. Indications for LVR were external RP ( $n=36)$, internal RP or symptomatic rectocele $(n=157)$ or a combination of symptomatic rectocele and enterocele $(n=40)$. Mean age and follow-up were 62 years (range 22-89) and 30 months (range 5-83), respectively. Response rate was 64\% (150 patients). The complication rate was $4.6 \%$ (11 complications). A significant reduction in symptoms of constipation or obstructed defecation syndrome was reported (53\% of patients before vs. $19 \%$ after surgery, $\mathrm{P}<0.001)$. Mean CCCS during follow-up was 8.1 points (range $0-23, S D \pm 4.3$ ). Incontinence was reported in 138 (59\%) of the patients before surgery and in 32 (14\%) of the patients after surgery, indicating a significant reduction $(P<0.001)$. Mean CCIS was 6.7 (range $0-19$, SD \pm 5.2) after surgery.

\section{Conclusion}

A significant reduction of incontinence and constipation or obstructed defecation syndrome after LVR was observed in this large retrospective study. LVR therefore appears a suitable treatment for RP and rectocele with and without associated enterocele. 


\section{INTRODUCTION}

Pelvic organ prolapse is a common disorder. The posterior compartment of the pelvic floor, the rectum, is often involved in this multi-organ problem[1]. The cause of associated symptoms such as incontinence, constipation and the obstructed defecation syndrome (ODS) can be various, including external and internal rectal prolapse (intussusception), large rectoceles and enteroceles.

Laparoscopic ventral rectopexy (LVR) is the most popular technique in Europe for the treatment of external and internal rectal prolapse [2-4]. Furthermore, it has been recommended for the treatment of large rectoceles [5]. During this procedure a mesh is placed between the anterior rectal wall and the posterior vaginal wall thereby reinforcing the rectovaginal septum. Most available papers on LVR describe significant improvement of functional symptoms with small complication rates [2,3,5-9]. These studies have been conducted in small patient series with a short follow-up. The aim of this study was to determine the functional results of all patients after LVR in our center up to 2011.

\section{PATIENTS AND METHODS}

\section{Study design}

This study comprised a retrospective analysis of all consecutive patients who underwent LVR for internal or external rectal prolapse and symptomatic rectocele (with or without enterocele) between 2004 and 2011. All patients underwent operation in a large teaching hospital in the Netherlands by one of two experienced pelvic floor surgeons. All patients who underwent primary surgery were included for analysis. Demographics, medical history, surgical and follow-up details of patients were collected from their medical records and gathered into a database.

\section{Evaluation and surgical technique}

Surgery was performed after work-up involving history, physical examination and radiological evaluation (dynamic MRI and/or evacuation proctography) of the pelvic floor with intra-vaginal and intra-rectal contrast enema. All patients were discussed in a multidisciplinary meeting consisting of dedicated gynecologists, urologists, radiologists, pelvic floor physical therapists and pelvic floor surgeons.

An external rectal prolapse (Oxford classification grade $V$ ) was an absolute indication for LVR. Furthermore, a history of constipation and/or fecal incontinence in combination with an Oxford grade III or IV internal rectal prolapse was an indication for surgery. Surgery was also performed in patients with similar functional symptoms in combination with an anterior rectocele, defined as $>2 \mathrm{~cm}$ bulging of the anterior rectal wall during physical examination (or objectified on dynamic MRI or evacuation proctography). 
Pelvic floor biofeedback therapy was started in all patients with an internal rectal prolapse or a rectocele prior to surgery. For patients with an external rectal prolapse, biofeedback therapy was initiated only if estimated to be useful, e.g. in the case of a concomitant pelvic floor descent.

The LVR procedure was carried out as described by D'Hoore and Penninckx [3]. Instead of a Marlex mesh (Bard, Crawley, UK), either a Hi-TEC mesh (Textiles HiTec, Labastide-Rouairoux, France) (until mid-2007) or a Prolene mesh (Ethicon Inc., Johnson \& Johnson, Hamburg, Germany) (from mid-2007 and on) was used. This switch in supplier and mesh was caused by new policies in stock acquisition, not for any medical or surgical reason. Proximal fixation upon the sacral promontory was performed with either titanium tacks (Autosuture Protack $5 \mathrm{~mm}$, Covidien, Mansfield, Massachusetts, USA) or one titanium $2 \mathrm{~mm} \times 8$ mm screw (Karl Storz, Tuttlingen, Germany), according to the surgeons' preferences.

\section{Questionnaire}

After informed consent, patients were asked to complete a questionnaire regarding functional results (constipation and incontinence). This questionnaire included the Cleveland Clinic Incontinence Score (CCIS) [10] and the Cleveland Clinic Constipation Score (CCCS) [11]. The CCIS and CCCS were translated into the Dutch language and underwent cultural adaptation according to the guidelines of the International Society of Pharmacoeconomics and Outcomes [12].

\section{Outcome parameters}

Symptoms of incontinence and constipation or ODS before and after surgery were set as main outcome parameters of this study. The Rome II criteria were used for routine preoperative and postoperative assessment of constipation and ODS. Fecal incontinence was defined as the involuntary loss of solid or liquid stool once or more during the last month.

Constipation/ODS was objectified with the CCCS (range $0-30$; a score of 30 is severe symptoms of constipation; a score > 15 is regarded as constipation). The CCIS (range 0-20; 20 is complete incontinence) was used for evaluation of incontinence. Complications were classified according to the Clavien-Dindo (CD) classification [13].

\section{Statistical Analysis}

Statistical analysis was performed using the Statistical Package for the Social Sciences, version 17.0 (SPSS Inc., Chicago, Illinois, USA). Patient data are presented as percentage or as mean \pm SD and range for all numerical variables. For the descriptive analyses, McNemar tests were used to evaluate differences in percentages. $\mathrm{P}<0.05$ was considered significant. 


\section{RESULTS}

\section{Patients and follow-up characteristics}

A total of 245 consecutive patients ( 234 women and 11 men) underwent LVR between 2004 and 2011. Twelve patients (5\%) died during the follow-up period due to causes unrelated to the LVR procedure and were therefore not analyzed. The remaining 233 patients ( 224 women, nine men) were included in the study and received a questionnaire. In total, 150 patients (64\%) completed and returned the questionnaire, 39 patients (17\%) were contacted but refused participation [for various reasons: no interest in participation $(n=12)$, deeming themselves too old for the questionnaire $(n=3)$, embarrassment $(n=2)$, miscellaneous/other reasons $(n=22)]$. A total of 44 patients $(19 \%)$ were lost to follow-up; addresses could not be retrieved.

General patient characteristics are presented in Table 1. Mean age at surgery was 62 years (range 22-89). The mean duration of follow-up at the time of the questionnaire was 30 months (range 5-87, SD 20.4).

Table 1 Baseline characteristics

\begin{tabular}{lc}
\hline Mean age & Total cohort $\mathbf{( n = 2 3 3 )}$ \\
Sex & 62 (range 22-89) \\
$\quad$ female & $224(96 \%)$ \\
$\quad$ male & $9(4 \%)$ \\
Mean parity (nr children) & $3($ range $0-10)$ \\
Prior abdominal/pelvic surgery & $121(52 \%)$ \\
Mean follow up in months & $30($ range $5-87)$ \\
Mean admission in days & $5($ range 3-30) \\
Post operative complications & $11(4.6 \%)$ \\
\hline
\end{tabular}

\section{Indication, operation, recurrence and complications}

Thirty-six patients (15\%) were operated on because of an external rectal prolapse, 157 patients (68\%) because of symptomatic rectocele or internal rectal prolapse and 40 patients (17\%) due to a symptomatic rectocele and/or internal rectal prolapse in combination with an enterocele.

In six patients a conversion to laparotomy was required due to extensive adhesions as a result of previous abdominal and pelvic surgery. They all underwent an open ventral rectopexy. 
No intra-operative complications occurred. The complication rate after surgery was $4.6 \%$ (11 patients) and included myocardial ischemia $(n=3)$ requiring admission on a specialized cardiac care unit (CD grade IVd). Two patients suffered from mesh infection complicated by discitis at the site of proximal mesh fixation. Antibiotic treatment was started and avoided surgical re-intervention in one (CD grade II), while the other patient needed revisional surgery. During operation, the infected mesh was removed, a temporary loop colostomy was situated and antibiotics were started (CD grade IIIb-d). One patient suffered from urinary retention, requiring a temporary urinary catheter (CD grade I). All remaining complications were CD grade II and consisted of urinary tract infections $(n=4)$ and one pneumonia. No mortality occurred. Mean hospital stay was 5 days (3-30, including the day of admission and discharge). Six patients $(2.6 \%)$ underwent revisional surgery during follow-up because of recurrence of (internal) rectal prolapse.

\section{Functional results}

Before surgery, 123 patients (53\%) reported symptoms of constipation or ODS. During follow-up, a significant overall reduction $(P<0.05)$ in these symptoms was found: 44 patients (19\%) encountered persisting constipation or ODS. In Table 2, preoperative and postoperative functional results per indication are depicted. For all indications, a significant reduction in ODS/constipation was observed. A total of five patients (2\%) encountered new onset constipation/ ODS after surgery. Mean CCCS during follow-up was 8.1 points (range $0-23$, SD \pm 4.3 ) out of 30 . Four patients ( $2.7 \%$ of respondents) had a score $>15$, which indicates significant constipation.

Before surgery, 138 patients (59\%) experienced symptoms of incontinence. After surgery, incontinence was encountered in 32 patients $(14 \%, P<0.05)$. A significant reduction in incontinence rates was observed for all indications, as can be seen in Table 2. In one patient (0.5\%), new onset incontinence was reported after operation. A mean CCIS of 6.7 (range $0-19$, SD \pm 5.2 ) out of 20 was determined after operation; $50 \%$ of respondents had a CCIS of $\leq 4$. 
Table 2 Functional results per indication

\begin{tabular}{|c|c|c|c|}
\hline Indication & $\begin{array}{c}\text { Pre-op. } \\
\text { incontinence }\end{array}$ & $\begin{array}{l}\text { Post-op. } \\
\text { incontinence }\end{array}$ & P-value \\
\hline $\begin{array}{l}\text { External rectal } \\
\text { prolapse }(n=36)\end{array}$ & $21(58 \%)$ & $5(14 \%)$ & $<0.001$ \\
\hline $\begin{array}{l}\text { Internal rectal prolapse / recto- } \\
\text { cele }(\mathrm{n}=157)\end{array}$ & 92 (59\%) & 20 (13\%) & $<0.001$ \\
\hline \multirow{2}{*}{$\begin{array}{l}\text { Combination rectocele and } \\
\text { enterocele }(n=40)\end{array}$} & 25 (63\%) & $7(18 \%)$ & $<0.001$ \\
\hline & $\begin{array}{c}\text { Pre-op. } \\
\text { constipation }\end{array}$ & $\begin{array}{l}\text { Post-op. } \\
\text { constipation }\end{array}$ & P-value \\
\hline $\begin{array}{l}\text { External rectal } \\
\text { prolapse }(n=36)\end{array}$ & 19 (53\%) & $8(22 \%)$ & 0.01 \\
\hline $\begin{array}{l}\text { Internal rectal prolapse / recto- } \\
\text { cele }(\mathrm{n}=157)\end{array}$ & $80(51 \%)$ & $27(17 \%)$ & $<0.001$ \\
\hline $\begin{array}{l}\text { Combination rectocele and } \\
\text { enterocele }(n=40)\end{array}$ & $24(60 \%)$ & $9(23 \%)$ & $<0.001$ \\
\hline
\end{tabular}

\section{Discussion}

LVR is an established technique for the treatment of rectal prolapse and has been described for the treatment of rectoceles as well [2-9]. As currently available studies tend to describe relatively small cohorts of patients, the aim of this study was to determine the influence of LVR on symptoms in a larger series of patients. A significant reduction in incontinence and constipation was found for patients with an external rectal prolapse, internal rectal prolapse or symptomatic rectocele and for patients with a combination of rectocele and enterocele. These results were accompanied by a low rate of complications.

For external rectal prolapse, similar results in reduction of incontinence and constipation are observed in the current study as in previous studies in the literature $[2,6]$.

The current literature on LVR for internal rectal prolapse and symptomatic rectocele is limited, especially with regard to functional outcomes. Two studies focus on symptomatic rectocele and internal rectal prolapse; both found a significant reduction of symptoms of ODS/constipation in small cohorts of patients (75 and 41 patients, respectively) after a median of 12 months follow-up $[5,7]$. Collinson et al. also focused on improvement of incontinence and found significant reduction of incontinence after a mean follow-up of 12 months [7]. In addition to these studies, the current study also found a significant improvement in functional outcomes after a mean follow-up of 30 months. 
Successful symptomatic outcome was not reported in all patients: $19 \%$ and $14 \%$ of patients respectively reported ongoing symptoms of constipation or incontinence. The cause of incontinence and constipation is regularly multifactorial and the patients who did not improve after surgery may have had other underlying factors causing symptoms of incontinence and constipation, such as for example anal sphincter failure or colonic transit disorders [14, 15]. Improved continence and constipation in patients after LVR seems to be caused by restored anatomy, probably resulting in a better function of the rectum, better sensitivity for faeces in the rectum and less bulging of the rectal wall, causing ODS. Exact mechanisms, however, are unknown. An important future goal is therefore to determine predicting factors for success or failure of LVR in patients with an external rectal prolapse, internal rectal prolapse or rectoceles. New onset constipation as a result of kinking of the redundant sigmoid after rectopexy has been described after posterior rectopexy $[16,17]$. In this study, new onset constipation was recorded in $2 \%$ of patients, which is in accordance with current literature [6].

A recent safety notification of the US Food and Drug Administration concerning pain, mesh infections and mesh erosion through the vagina after mesh implant for pelvic organ prolapse surgery in 2011 led to a discussion about the use of meshes for this indication $[18,19]$. These reports, however, concern transvaginal positioned meshes. Reported mesh related complications in the current study consisted of only two mesh infections. These cases are the only reported cases of mesh infection after LVR until now and have been described previously by our group [20]. Therefore, the occurrence of mesh related complications after LVR is limited, despite the position of the mesh at the site of the rectovaginal septum. Probably, because of differences in surgical approach abdominal positioned meshes offer fewer safety issues and complications compared with transvaginal meshes. We also report a low percentage of re-operation due to failure as a whole (2.8\%).

The cross-sectional design of this study has some methodological disadvantages. It was only possible to gather preoperative incidences of symptoms but no actual severity scores, as we did not routinely perform the CCCS and CCIS questionnaires during evaluation. Comparison of validated scores before and after surgery was consequently impossible. Furthermore, our response rate was $65 \%$, caused by refusal to participate and loss to follow-up in this relatively elderly patient population. This loss of patients in our follow-up might have influenced results, as it is imaginable that patients who are unsatisfied by the results of the procedure are less willing to fill out questionnaires. This burden is closely related to questionnaire studies and its exact impact cannot be determined. Nevertheless, the current study provides an adequate overview of 
complications, recurrences and functional results after LVR in a large cohort of patients with a mean follow-up of 30 months.

In conclusion, a significant reduction of incontinence and constipation was observed after LVR for patients with a rectal prolapse and/or symptomatic rectocele. These outcomes support the application of LVR for these indications. 


\section{REFERENCES}

1 Elneil S. Complex pelvic floor failure and associated problems. Best Pract Res Clin Gastroenterol 2009; 23: 555-73.

2 D’Hoore A, Cadoni R, Penninckx F. Long-term outcome of laparoscopic ventral rectopexy for total rectal prolapse. Br J Surg 2004; 91: 1500-5.

3 D’Hoore A, Penninckx F. Laparoscopic ventral recto(colpo)pexy for rectal prolapse: surgical technique and outcome for 109 patients. Surg Endosc 2006; 20: 1919-23.

4 Formijne Jonkers HA, Draaisma WA, Wexner SD et al. Evaluation and surgical treatment of rectal prolapse: an international survey. Colorectal Dis 2013; 15: 115-9.

5 Wong MT, Abet E, Rigaud J, Frampas E, Lehur PA, Meurette G. Minimally invasive ventral mesh rectopexy for complex rectocoele: impact on anorectal and sexual function. Colorectal Dis 2011; 13: e320-6.

6 Boons P, Collinson R, Cunningham C, Lindsey I. Laparoscopic ventral rectopexy for external rectal prolapse improves constipation and avoids de novo constipation. Colorectal Dis 2010; 12: 526-32.

7 Collinson R, Wijffels N, Cunningham C, Lindsey I. Laparoscopic ventral rectopexy for internal rectal prolapse: short-term functional results. Colorectal Dis 2010; 12: 97-104.

8 van den Esschert JW, van Geloven AA, Vermulst N, Groenedijk AG, de Wit LT, Gerhards MF. Laparoscopic ventral rectopexy for obstructed defecation syndrome. Surg Endosc 2008; 22: 2728-32.

9 Wijffels N, Cunningham C, Dixon A, Greenslade G, Lindsey I. Laparoscopic ventral rectopexy for external rectal prolapse is safe and effective in the elderly. Does this make perineal procedures obsolete? Colorectal Dis 2011; 13: 561-6.

10 Jorge JM, Wexner SD. Etiology and management of fecal incontinence. Dis Colon Rectum 1993; 36: 77-97.

11 Agachan F, Chen T, Pfeifer J, Reissman P, Wexner SD. A constipation scoring system to simplify evaluation and management of constipated patients. Dis Colon Rectum 1996; 39: 681-5.

12 Wild D, Grove A, Martin M et al. Principles of Good Practice for the Translation and Cultural Adaptation Process for Patient-Reported Outcomes (PRO) Measures: Report of the ISPOR Task Force for Translation and Cultural Adaptation. Value Health 2005; 8: 94-104.

13 Dindo D, Demartines N, Clavien PA. Classification of surgical complications: a new proposal with evaluation in a cohort of 6336 patients and results of a survey. Ann Surg 2004; 240: 205-13.

14 Hayden DM, Weiss EG. Fecal incontinence: etiology, evaluation, and treatment. Clin Colon Rectal Surg 2011; 24: 64-70.

15 Rao SS. Constipation: evaluation and treatment. Gastroenterol Clin North Am 2003; 32: 659-83.

16 Mckee RF, Lauder JC, Poon FW, Aitchison MA, Finlay IG. A prospective randomized study of abdominal rectopexy with and without sigmoidectomy in rectal prolapse. Surg Gynecol Obstet 1992; 174: 145-8. 
17 Sayfan J, Pinho M, Alexander-Williams J, Keighley MR. Sutured posterior abdominal rectopexy with sigmoidectomy compared with Marlex rectopexy for rectal prolapse. Br J Surg 1990; 77: 143-5.

18 Rogers RG. To mesh or not to mesh: current debates in prolapse repair fueled by the U.S. Food and Drug Administration Safety Notification. Obstet Gynecol 2011; 118: 771-3.

19 Steinberg AC. Use of vaginal mesh in the face of the recent FDA warnings and litigation. Am J Obstet Gynecol 2011; 204: e10-1.

20 Draaisma WA, van Eijck MM, Vos J, Consten EC. Lumbar discitis after laparoscopic ventral rectopexy for rectal prolapse. Int J Colorectal Dis 2011; 26: 255-6. 


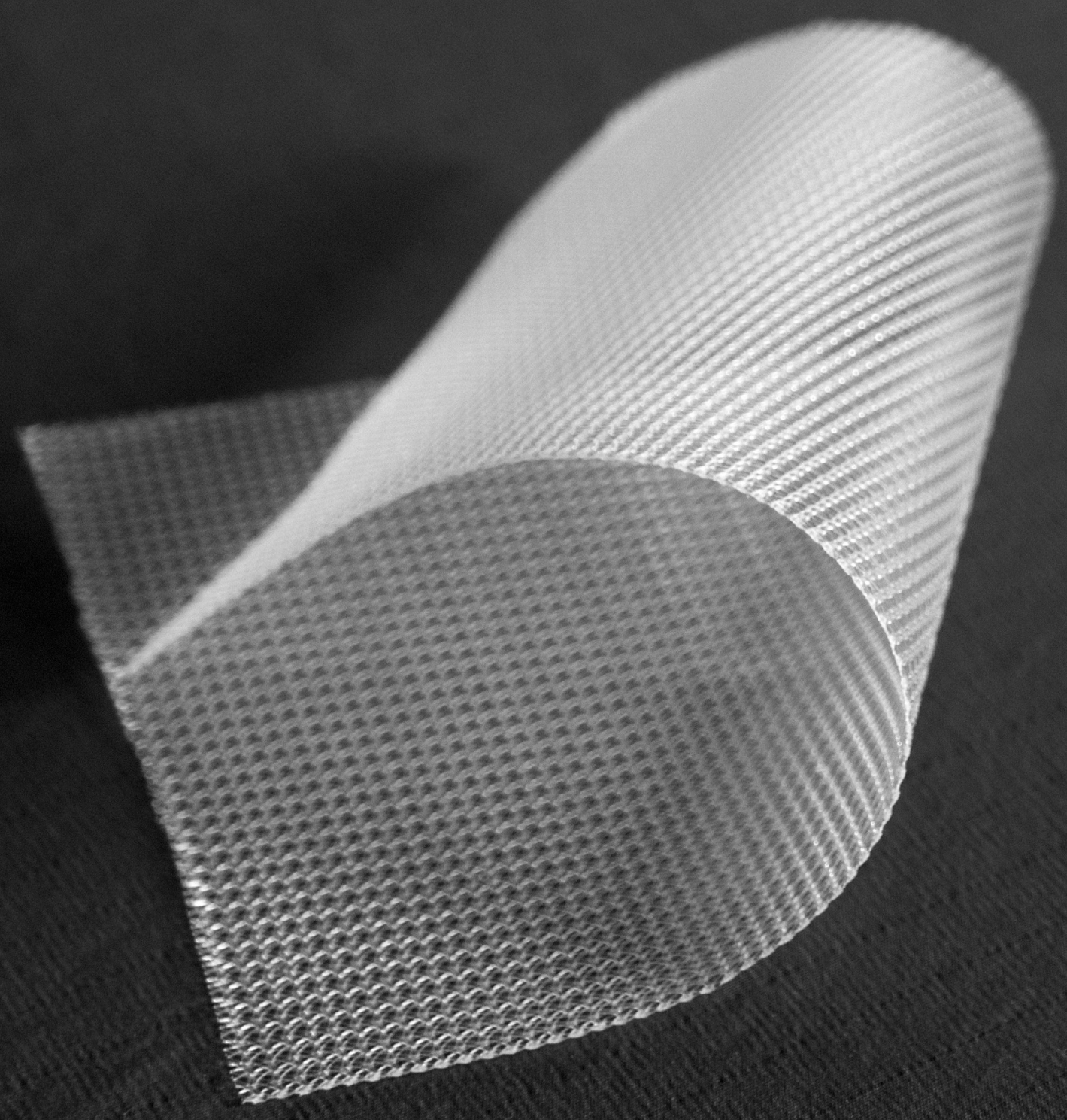




\section{Chapter 4}

\section{Impact of rectopexy on Sexual Function; A Cohort Analysis}


Alm

Laparoscopic ventral rectopexy (LVR) is an established surgical technique for the treatment of both rectal prolapse and symptomatic rectoceles. It is, however, not known whether LVR influences sexual function (SF). The aim of this study was, therefore, to determine the impact of this procedure on the SF of patients.

\section{METHODS}

All female patients after LVR procedure in a single institution were identified and were sent a questionnaire concerning SF. This addressed sexual activity, satisfaction, preoperative SF, and the impact of surgery on SF. Furthermore, the PISQ-12 validated sexual functioning questionnaire was sent to all female patients.

\section{RESULTS}

A total of 217 patients were sent a questionnaire. These patients underwent LVR for rectal prolapse, symptomatic rectocele, or enterocele between 2004 and 2011. Mean age was 62 years (range 22-89). Mean follow-up was 30 months (range 5-83). Response rate was $64 \%$ (139 patients). The number of sexual active patients dropped from 71 to $54 \%$ after surgery. The number of patients being satisfied with their SF remained relatively equal; $91 \%$ of patients before and $85 \%$ of patients after surgery. Forty-three percent of patients stated that the LVR procedure did not influence their sexual function, in 16 $\%$ of patients, the procedure positively influenced their SF, and in $13 \%$ of respondents, SF decreased after surgery. The mean PISQ-12 score postoperatively was 34 out of 48 .

\section{CONCLUSIONS}

The impact of LVR on SF of patients seems limited in this cross-sectional study in a large cohort of patients. 


\section{INTRODUCTION}

Laparoscopic ventral rectopexy (LVR) is an established surgical technique for the treatment of several pelvic floor disorders, including internal and external rectal prolapse and complex rectoceles [1-5]. During this procedure, a mesh is placed between the anterior rectal wall and the posterior vaginal wall, thereby reinforcing the rectovaginal septum. This technique avoids full rectal mobilization, and thereby spares the autonomic nerves of the pelvic floor [2]. Several articles have described the safety and effectiveness of LVR. In general, these studies demonstrate significant improvement of incontinence and constipation, the main symptoms of patients with a rectal prolapse or symptomatic rectocele [1-6]. In general, the influence of surgical corrections of pelvic floor prolapse on sexual function (SF) has been investigated widely. These studies demonstrate conflicting results; some studies show improved SF, while others describe deterioration, probably caused by factors like mesh exposure and tissue contraction around the mesh [7-9]. At present, only one small cohort study describing SF after LVR is available. This study determined a significant decrease of intercourse dyspareunia and sexual difficulties after surgery [5]. However, nowadays, surgeons are often questioned whether LVR influences the SF of patients after operation, especially due to debates in both medical literature and the media about the use of meshes in prolapse surgery. This started after an safety notification of the US Food and Drug Administration concerning dyspareunia and mesh erosion trough the vagina after mesh implant for pelvic organ prolapse surgery in 2011 [10,11]. The aim of this study was therefore to evaluate the SF of patients after LVR and to compare this with the SF before surgery.

\section{METHODS}

\section{Study design}

This study comprised a cross-sectional analysis of all consecutive, female, patients who underwent LVR for internal or external rectal prolapse, complex rectocele, or symptomatic enterocele, between January 2004 and February 2011. All patients were operated in a large teaching hospital in the Netherlands by one of two experienced pelvic floor surgeons (IB and EC). All patients who underwent primary surgery were included for analysis. Characteristics, demographics, medical history, operation, and follow-up details of all patients were collected from their medical records and retrospectively gathered into a database. 


\section{Treatment and Surgical technique}

Surgery was performed after extensive workup involving anamnesis, physical examination, and radiological work up (dynamic MRI of the pelvic floor with intravaginal and intrarectal contrast enema). All patients were discussed in a multidisciplinary meeting consisting of dedicated gynecologists, urologists, radiologists, pelvic floor physical therapists, and pelvic floor/colorectal surgeons. A sexologist was consulted on indication. Pelvic floor biofeedback therapy was started if estimated useful. The LVR procedure was carried out as described by D'Hoore et al. [2]. Instead of a Marlex (Bard, Crawley, UK) mesh, either a Hi-Tec mesh (Textiles Hi-Tec, Labastide-Rouairoux, France) or Prolene (Ethicon inc., Johnson \& Johnson, Hamburg, Germany) mesh was used. Proximal fixation upon the sacral promontory was performed with either titanium, tacks (Autosuture ProTack $5 \mathrm{~mm}$, Covidien, USA), or one titanium, $2 \times 8 \mathrm{~mm}$, screw (Karl Storz, Tuttlingen, Germany).

\section{Questionnaire}

All identified patients were asked to complete a questionnaire, sent by mail, regarding their sexual function. This questionnaire consisted of questions concerning preoperative and present sexual activity, satisfaction, pain during sexual activities, and the influence of LVR on their sexual function. The questionnaire furthermore consisted of the Pelvic Organ Prolapse-Urinary Incontinence Sexual Function Questionnaire (PISQ-12), a validated questionnaire to determine the SF of women with pelvic organ prolapse symptoms [12]. Scores for PISQ-12 range from $0-48$, with a higher score indicating a better sexual function. The identical Dutch translation of the PISQ-12 was used as in previous Dutch studies $[13,14]$.

\section{Ethics}

This study was carried out in accordance with the ethical standards of the Declaration of Helsinki (1975), as revised in 1983. All patients gave informed consent prior to inclusion in this study.

\section{Statistical Analysis}

Statistical analysis was performed using statistical package for the social science, version 17.0 (SPSS Inc., Chicago, IL, USA). Patient data are presented as percentage or as mean \pm SD and range for all numerical variables.

\section{Outcome parameters}

Outcomes on pre- and postoperative sexual activity, satisfaction, and the influence of LVR on their sexual function were scored, as well as the PISQ-12 score during follow-up. 


\section{RESULTS}

\section{Patient characteristics}

A total of 234 female patients were identified who underwent LVR. Eleven patients died during the follow-up period due to causes unrelated to the LVR procedure. Five patients had undergone surgery for recurrence of the operation indication and were therefore excluded. Another patient had to undergo revisional surgery because of an infected mesh with subsequent complaints. This mesh was removed during surgery, and a temporary loop colostomy was situated. This patient was also excluded. The remaining 217 patients were included in the study and received a printed questionnaire. A total of 139 patients (64\%) completed and returned the questionnaire, 37 patients (17\%) were contacted but refused to participate (based on various reasons: no interest in participation ( $n=12)$, bad (sexual) experiences $(n=4)$, deeming themselves too old for the questionnaire $(n=3)$, embarrassment $(n=2)$, and miscellaneous/other reasons $(n=16))$. A total of 41 patients $(19 \%)$ were lost to follow-up, addresses could not be retrieved. In Table 1 general patient characteristics are presented. Mean age at time of surgery was 62 years (range 22-89). The mean follow-up after surgery at the time of the questionnaire was 30 months (range 5-87, SD 20.4).

Table 1 Baseline characteristics

\begin{tabular}{lc}
\hline & Total cohort $\mathbf{( n = 2 1 7 )}$ \\
\hline Mean age (range) & $62(22-89)$ \\
Mean parity (range) & $3(0-10)$ \\
Mean follow up in months (range) & $30(5-87)$ \\
Mean admission in days (range) & $5(3-30)$ \\
Post-operative complication rate & $\mathrm{N}=10(4.6 \%)$ \\
\hline
\end{tabular}

\section{Indication, operation and complications}

Thirty-two patients (14.7\%) were operated because of an external rectal prolapse, 145 patients $(66.8 \%)$ because of symptomatic rectocele or internal rectal prolapse, and 40 patients ( $18.4 \%$ ) due to a symptomatic rectocele and/or internal rectal prolapse in combination with an enterocele. In five patients, a conversion to laparotomy was required due to extensive adhesions as a result of previous abdominal and pelvic surgery. They all underwent open ventral rectopexy. There were no intraoperative complications. Postoperative complication rate was $4.6 \%$ (ten patients) and consisted of myocardial ischemia $(n=3)$, 
urinary tract infections $(n=4)$, urinary retention $(n=1)$, and pneumonia $(n=1)$. One patient suffered from mesh infection complicated by discitis at the site of proximal mesh fixation. Antibiotic treatment was started and avoided surgical re-intervention. No mortality occurred. Average admittance was 5 days (3-30, including day of admission and discharge).

\section{Sexual function: general questions}

A total of $98(71 \%)$ patients confirmed to be sexually active to some extent before surgery. This was $54 \%$ (75 patients) at the moment of follow-up. Preoperatively, $90.8 \%$ of sexual active patients indicated to be less or more satisfied with their sexual function versus $84.7 \%$ of patients after surgery. Exact numbers per grade of satisfaction can be found in Table 2 .

A total of 60 patients ( $43 \%$ ) indicated that the LVR had not influenced their SF, $13 \%$ indicated that their SF deteriorated because of the procedure, while $16 \%$ specified a better SF as a results of the operation.

Table 2. Rates of satisfaction about sexual function

\begin{tabular}{lcc}
\hline Satisfaction about SF & Preoperative & Postoperative \\
\hline Some satisfaction & $30.6 \%$ & $20.0 \%$ \\
Moderate satisfaction & $42.9 \%$ & $45.9 \%$ \\
Total satisfaction & $17.3 \%$ & $18.8 \%$ \\
Total & $90.8 \%$ & $84.7 \%$ \\
\hline
\end{tabular}

\section{Sexual function: PISQ-12}

All patients who indicated to be sexually active at the moment of follow-up (75 patients, $54 \%$ ) completed and returned the PISQ-12. The mean PISQ-12 score at the moment of follow-up in our cohort was 34 out of 48 points (SD \pm 6.1 , range $20-42$ ).

\section{Discussion}

Laparoscopic ventral rectopexy is a popular surgical technique for the treatment of both internal and external rectal prolapse and symptomatic rectoceles, especially in European countries. Although the safety and functional results of this procedure have been described frequently, current literature on sexual function is lacking. Therefore, the aim of this study was to determine the influence of LVR on the SF in a large cohort of patients. In general, this study demonstrated that the number of sexually active patients decreased from $71 \%$ before surgery to $54 \%$ at the moment of FU, while satisfaction rates remained 
relatively unchanged. This decrease in sexual activity rate is influenced by the further aging of the population. Furthermore, several patients noted that their sexual activity unfortunately was stopped, since their partner died. Similar decreases in the number of sexually active patients are observed in other articles that describe SF before and after surgery for rectoceles, including the single other study that described SF of patients after LVR [5, 7].Due to the relatively large number of patients in our cohort it was possible to determine the influence of LVR on sexual function. More than $40 \%$ of sexually active patients indicated that the LVR procedure did not alter their SF, while $16 \%$ of patients experienced an improved SF. Deterioration in SF during FU was experienced by $13 \%$ of patients. The remainder of patients could not answer this question or were not sexually active. Overall, the impact of LVR on SF based on results fund in the current study seems limited. The only known study illustrating SF after LVR merely used standardized and simple questions to evaluate the SF in their study. This study comprised of 41 patients and determined a significant decrease in sexual difficulties and intercourse dyspareunia after operation [5]. In our cohort with 226 patients, we have added the validated PISQ-12 questionnaire to evaluate SF, demonstrating a mean score of 34 points. Comparable PISQ-12 scores can be found in the various articles describing SF after surgical repair of (advanced) pelvic organ prolapse and repair of rectoceles (using different surgical techniques involving the placement of a mesh). In general, these studies describe postoperative PISQ-12 scores between 34 and 37 points [7, 13, $15]$. The PISQ-12 score for the general population is $36.4[12,16]$. Due to the retrospective design, it was not possible to determine the PISQ-12 score before surgery. Comparison of this score before and after LVR was therefore not executable. However, due to validation studies of the PISQ-12, and the use of this questionnaire in other studies, we know that the SF of our cohort of patients does not differ from the SF of the general population and the SF of patients after various procedures of pelvic floor surgery.

In conclusion, the impact of LVR on the SF of patients seems limited in this study. As possible effects on the SF are often questioned by patients during preoperative consults, this information is regarded valuable. Currently, we conduct a prospective study to determine the impact of LVR on the SF to underline the results of this study. 


\section{REFERENCE LIST}

1. D'Hoore A, Cadoni R, Penninckx F (2004) Long-term outcome of laparoscopic ventral rectopexy for total rectal prolapse. Br J Surg 91(11):1500-1505

2. D’Hoore A, Penninckx F (2006) Laparoscopic ventral recto(colpo)pexy for rectal prolapse: surgical technique and outcome for 109 patients. Surg Endosc 20(12):1919-1923

3. Collinson R, Wijffels N, Cunningham C, Lindsey I (2010) Laparoscopic ventral rectopexy for internal rectal prolapse: short-term functional results. Colorectal Dis 12((2):97-104

4. van den Esschert JW, van Geloven AA, Vermulst N et al (2008) Laparoscopic ventral rectopexy for obstructed defecation syndrome. Surg Endosc 22(12):2728-2732

5. Wong MT, Abet E, Rigaud J et al (2011) Minimally invasive ventral mesh rectopexy for complex rectocele: impact on anorectal and sexual function. Colorectal Dis 13(10):e320-e326

6. Wijffels N, Cunningham C, Dixon A, Greenslade G, Lindsey I (2011) Laparoscopic ventral rectopexy for external rectal prolapse is safe and effective in the elderly. Does this make perineal procedures obsolete? Colorectal Dis 13(5):561-566

7. Paraiso MF, Barber MD, Muir TW, Walters MD (2006) Rectocele repair: a randomized trial of three surgical techniques including graft augmentation. Am J Obstet Gynecol 195(6):1762-1771

8. Klinge U, Klosterhalfen B, Muller M, Ottinger AP, Schumpelick V (1998) Shrinking of polypropylene mesh in vivo: an experimental study in dogs. Eur J Surg 164(12):965-969

9. Feiner B, Maher C (2010) Vaginal mesh contraction: definition, clinical presentation, and management. Obstet Gynecol 115(2 Pt 1):325-330

10. Rogers RG (2011) To mesh or not to mesh: current debates in prolapse repair fueled by the US Food and Drug Administration Safety Notification. Obstet Gynecol 118(4):771-773

11. Steinberg AC (2011) Use of vaginal mesh in the face of the recent FDA warnings and litigation. Am J Obstet Gynecol 204(6):e10-e11

12. Rogers RG, Coates KW, Kammerer-Doak D, Khalsa S, Qualls C (2003) A short form of the Pelvic Organ Prolapse/Urinary Incontinence Sexual Questionnaire (PISQ-12). Int Urogynecol J Pelvic Floor Dysfunct 14(3):164-168

13. Milani AL, Withagen MI (2011) The HS, Nedelcu-van dW, I, Vierhout ME. Sexual function following trocar-guided mesh or vaginal native tissue repair in recurrent prolapse: a randomized controlled trial. J Sex Med 8(10):2944-2953

14. Wild D, Grove A, Martin M et al (2005) Principles of good practice for the translation and cultural adaptation process for patient-reported outcomes (PRO) measures: report of the ISPOR task force for translation and cultural adaptation. Value Health 8(2):94-104

15. Geller EJ, Parnell BA, Dunivan GC (2011) Pelvic floor function before and after robotic sacrocolpopexy: one-year outcomes. J Minim Invasive Gynecol 18(3):322-327 
Impact of rectopexy on Sexual Function; A Cohort Analysis

16. Rogers RG, Kammerer-Doak D, Villarreal A, Coates K, Qualls C (2001) A new instrument to measure sexual function in women with urinary incontinence or pelvic organ prolapse. Am J Obstet Gynecol 184(4):552-558 


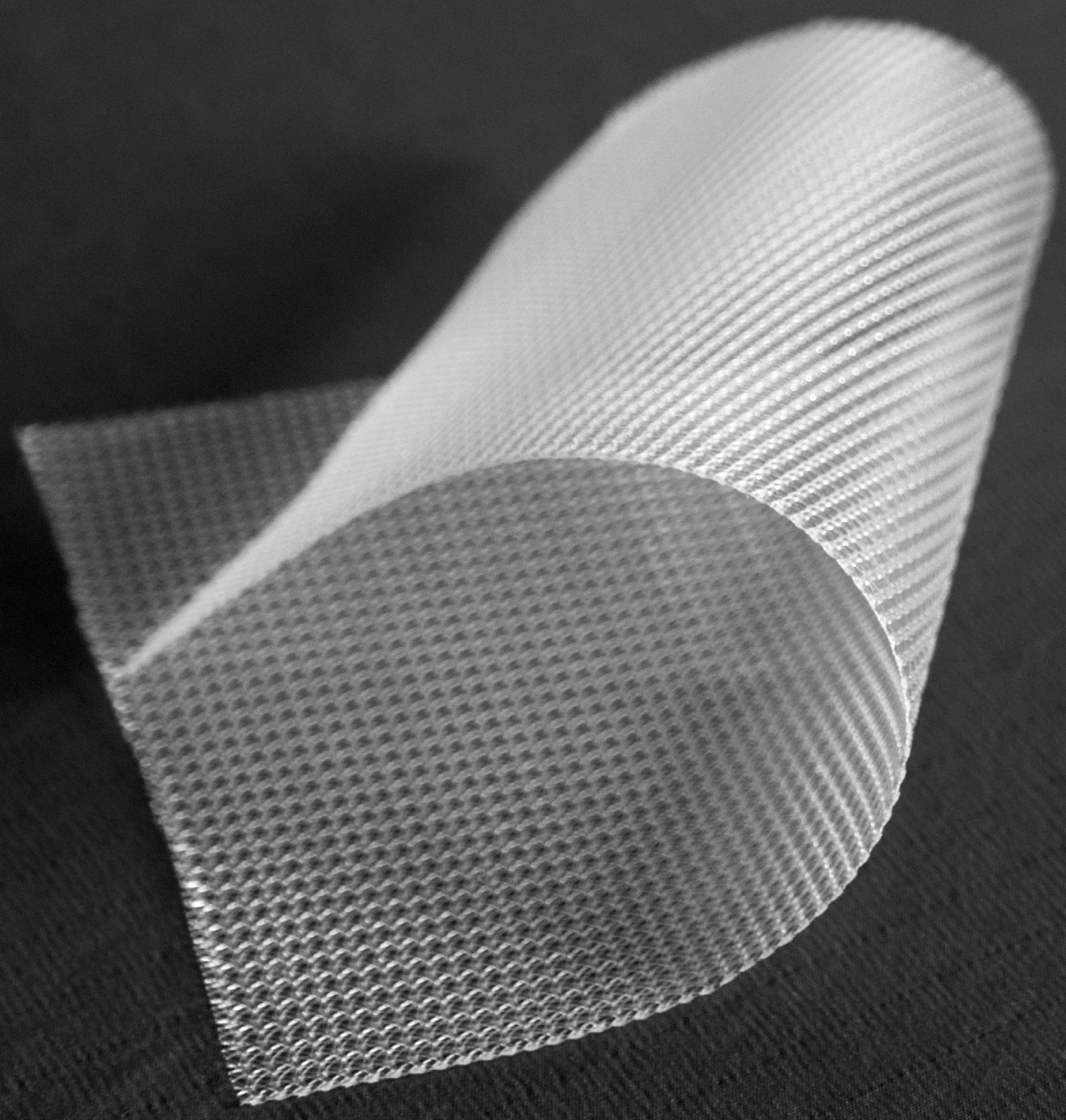




\section{Chapter 5}

\section{Laparoscopic resection rectopexy versus laparoscopic ventral rectopexy for complete rectal prolapse}


ABSTRACT

\section{Background}

Laparoscopic resection rectopexy (LRR) and laparoscopic ventral rectopexy (LVR) are favored for the treatment of rectal prolapse (RP) in the USA and Europe, respectively. This study aims to compare these two surgical techniques.

\section{Methods}

All patients who underwent LRR because of RP between 01-2000 and 01-2012 at Cleveland Clinic Florida (Weston, FL, USA) were identified and all relevant characteristics were entered in a database. This same analysis was also conducted for all patients who underwent LVR in the Meander Medical Center (Amersfoort, the Netherlands) between 01-2004 and 01-2012. These two cohorts were retrospectively compared with regard to complications, functional results and recurrence.

\section{Results}

Twenty-eight patients (all female, mean age: 50.1 years) were included in the LRR cohort at a mean followup of 57 (range 2-140; standard

deviation (SD) \pm 41.2 ) months. The LVR group consisted of 40 patients (36 females, 4 males) with a mean age of 67.0 years and a mean follow-up of 42 (range 2-82;SD \pm 23.8 ) months. A significant reduction in constipation was observed in both cohorts after surgery: $57 \%$ vs. $21 \%$ after LRR, $55 \%$ vs.23\% after LVR (both $\mathrm{P}<0.05$ ). The incidence of incontinence also significantly decreased in both groups; $15 \%$ after LVR (55\% before surgery) and 4\% after LRR (61\% before surgery). Direct comparison of these two techniques showed a trend to significance $(p=0.09)$. Significantly more complications occurred after LRR ( $n=9: 1$ major, 8 minor) then after $\operatorname{LVR}(n=3,2$ major, 1 minor) ( $p<0.05)$.

\section{Conclusions}

Both LVR and LRR are effective for the treatment of RP. Although both techniques offer significant improvements in functional symptoms, continence may be better after LRR. However LRR also had a higher complication rate then did LVR. 


\section{INTRODUCTION}

External rectal prolapse (RP) is the full thickness, circumferential intussusception of the rectal wall beyond the anal canal. It is a common disorder in the western world, occurring predominantly in middle aged and older women. The majority of patients with a RP suffer from symptoms of fecal incontinence and constipation, causing a significant negative impact on quality of life. Medications like bulking agents and biofeedback therapy of the pelvic floor might relieve symptoms; surgery is however the only definitive treatment for RP [1-3]. Many surgical techniques, both perineal and abdominal approaches, have been proposed for the treatment of RP during the last centuries [4]. Traditionally, abdominal techniques are used in fit patients, while anal or perineal techniques are reserved for the frail and elderly. Since the emergence of minimally invasive surgery, laparoscopic techniques for the treatment of RP have been applied in patients of all ages, including octogenarians. Not only has laparoscopic rectopexy proved to be safe and effective in patients of all ages it also offers a lower rate of postoperative surgical site infection and length of hospital stay [5-9]. A recent international survey among colorectal surgeons revealed that laparoscopic resection rectopexy (LRR) and laparoscopic ventral rectopexy (LVR) are the techniques preferred by surgeons in the United States of America and Europe, respectively [10].

No studies have been published in the literature comparing these two most popular techniques for the treatment of RP. As the results of prospective, comparative studies are awaited, the aim of the current study was to retrospectively compare the results of LRR and LVR for the treatment of external RP.

\section{MATERIALS AND METHODS}

\section{Study design and patients}

This study was designed as a retrospective, cross-sectional, comparative, double-cohort study of patients operated on for full thickness rectal prolapse.

One cohort consisted of all patients who underwent LRR at Cleveland Clinic Florida (CCF, Weston, FL., U.S.A.), between 01-2000 and 01-2012. The second cohort consisted of all patients who underwent LVR in the Meander Medical Center (MMC, Amersfoort, The Netherlands), from 01-2004 (when this technique was first performed in the MMC) until 01-2012.

Relevant demographic data, medical history, surgical details and follow-up characteristics of all patients were collected from their medical records and gathered into a database. Chart review was performed in both centers by the same researcher (HAFJ) in an identical fashion. After data gathering, results were compared. 


\section{Ethics}

This study was undertaken in accordance with the ethical standards of the Declaration of Helsinki (1975), as revised in 1983. The study protocol was reviewed and approved by the respective Institutional Review Boards of the MMC and CCF.

\section{Treatment and surgical technique}

Surgery was performed after extensive evaluation including medical history, physical examination and radiological assessment (dynamic magnetic resonance imaging (MRI) or cinedefecography of the pelvic floor with intravaginal and intrarectal contrast enema). All patients were discussed in a multidisciplinary meeting consisting of dedicated colorectal surgeons, gynecologists and urologists (in MMC), or colorectal surgeons and urogynecologists (in CCF). Radiologists and pelvic floor physical therapists were also included in the discussions as needed. Pelvic floor biofeedback therapy initiated prior to surgery was a matter of surgeon choice.

\section{Laparoscopic ventral rectopexy}

The LVR procedure is undertaken in an identical fashion as described by D'Hoore et al [11]. In addition, the rectovaginal plane was dissected all the way down to the level of the pelvic floor in all patients. Instead of a Marlex mesh (Bard, Crawley, UK), either a Hi-TEC mesh (Textiles Hi-Tec, Labastide-Rouairoux, France) or Prolene mesh (Ethicon inc., Johnson \& Johnson, Hamburg, Germany) is used. This mesh is cut to a strip of $3 \mathrm{~cm} . \times 15 \mathrm{~cm}$. and subsequently fixed to the ventral aspect of the distal rectum using at least six individual sutures. Proximal fixation upon the sacral promontory is performed with either titanium tacks (Autosuture Protack 5mm, Covidien, USA) or one titanium $2 \mathrm{~mm} \times 8$ $\mathrm{mm}$ screw (Karl Storz, Tuttlingen, Germany). In female patients the dorsal vaginal wall is thereafter fixed to the mesh traction free with two lateral sutures at the vaginal top before closing the peritoneum over the mesh.

\section{Laparoscopic resection rectopexy}

All patients are given preoperative mechanical and oral antibiotic bowel preparation, as well as perioperative parenteral antibiotics. The procedure is performed under general anesthesia with the patient in the supine modified lithotomy position. Three $12 \mathrm{~mm}$ ports are utilized: a camera port in the infraumbilical incision and two working ports, one in the right iliac fossa and one in the right upper quadrant, each through a horizontal stab wound. The entire left colon is mobilized with the $5 \mathrm{~mm}$ diameter ultrasonic scalpel: the splenic flexure is deliberately not mobilized. The entire rectum is mobilized into the levator muscles carefully separating the posterior vaginal wall from the anterior 
rectal wall. After full mobilization with division of the upper two thirds of the lateral ligaments a small Pfannenstiel incision is made through which a wound protector is placed. The blood vessels are then divided along the bowel wall to deliberately preserve the inferior mesenteric artery and vein and the superior rectal artery and vein. A point is selected at the descending sigmoid junction for placement of a purse string suture. Distally, a minimum of three and a maximum of six rectopexy sutures are placed through the upper portions of the divided lateral stalks and the presacral fascia and anterior sacral periostium. These 2-0 sutures are placed in a horizontal mattress configuration. Great care is taken not to injure the blood vessels in the divided stalks or in the bowel wall. The sutures are placed but not tied. The rectosigmoid junction is then divided taking care to insure that the anastomosis will not be in immediate proximity to any of the rectopexy sutures. After creation of the end- to- end anastomosis, the anastomosis is tested by endoscopic visualization including air insufflation during endoscopy. The rectopexy sutures are then secured, after which the flexible endoscopy with air insufflation is repeated to insure that the lumen was not narrowed and that the anastomosis remains air tight and completely hemostatic. A $19 \mathrm{~mm}$ suction drain is placed in the presacral space after which all incisions are closed.

\section{Admission and discharge}

All patients were admitted on the day of surgery. Discharge criteria were identical in both clinics and included tolerance of full diet, passage of stool, being able to walk properly and adequate oral analgesia.

\section{Outcome parameters}

The incidence of constipation / obstructed defecation syndrome (ODS) before surgery and during follow-up was retrospectively scored using the Rome II criteria. Fecal incontinence was also retrospectively scored and was defined as the involuntarily loss of solid or liquid stool, once or more per month.

Other endpoints were perioperative and postoperative complications, length of hospital stay and recurrences during follow-up. Complications were scored according to the Clavien-Dindo classification [12].

\section{Statistical analysis}

Statistical analysis was performed using Statistical Package for the Social Science, version 17.0 (SPSS Inc., Chicago, IL, USA). Patient data are presented as percentage or as mean \pm standard deviation (SD) and range for all numerical variables.

For the descriptive analyses, McNemar tests were used to evaluate differences in percentages. $P$ values $<0.05$ were considered significant. 


\section{RESULTS}

Patients and follow-up characteristics

Twenty-eight patients were included in the LRR cohort; and 40 patients in the LVR cohort. The mean age was significantly lower in the LRR cohort compared to the LVR group (50.1 vs. 67.0 years, respectively). The mean follow-up was longer in the LRR group (57 months) compared to the LVR cohort (42 months), although this was not significant. Baseline characteristics can be found in table 1a.

Table 1a Baseline and follow-up characteristics

\begin{tabular}{lccc}
\hline & $\begin{array}{c}\text { LVR cohort } \\
\mathbf{N = 4 0}\end{array}$ & $\begin{array}{c}\text { LRR cohort } \\
\mathbf{N = \mathbf { 2 8 }}\end{array}$ & P-value \\
\hline $\begin{array}{l}\text { Mean age in years (SD) } \\
\text { Range }\end{array}$ & $67.0( \pm 15.4)$ & $50.1( \pm 17.9)$ & 0.00 \\
Sex & $22-89$ & $18-76$ & \\
$\quad$ female & 36 & & \\
$\quad$ male & 4 & 28 & \\
Prior abdominal/pelvic surgery & $14(35 \%)$ & $8(29 \%)$ & 0.60 \\
$\quad$ including hysterectomy & $8(20 \%)$ & $3(11 \%)$ & 0.32 \\
Mean follow up in months (SD) & $42( \pm 23.8)$ & $57( \pm 41.2)$ & 0.067 \\
$\quad$ Range & $2-82$ & $2-140$ & \\
\hline
\end{tabular}

Table $\mathbf{1 b}$ Overview of length of admission and complications

\begin{tabular}{lccc}
\hline & $\begin{array}{c}\text { LVR cohort } \\
\mathbf{N = 4 0}\end{array}$ & $\begin{array}{c}\text { LRR cohort } \\
\mathbf{N = 2 8}\end{array}$ & P-value \\
\hline Mean admission in days (SD) & $5.29( \pm 2.5)$ & $5.43( \pm 2.4)$ & 0.82 \\
\multicolumn{1}{c}{ Range } & $3-17$ & $3-12$ & \\
Complications during surgery & $1(2.5 \%)$ & $2(7.1 \%)$ & 0.58 \\
$\begin{array}{l}\text { Postoperative complications } \\
\text { (see table 2) }\end{array}$ & $3(7.5 \%)$ & $9(32 \%)$ & 0.01 \\
\hline
\end{tabular}

\section{Admission, procedure and complications}

There was no significant difference in length of admission between the two cohorts: mean admission was 5.3 (range $3-17, \mathrm{SD} \pm 2.5$ ) days for the LVR group and 5.43 (range 3-12, SD \pm 2.4 ) days in the LRR group. For admission details see table $1 \mathbf{b}$. 
There were three intraoperative complications: one in the LVR group (2.5\%), two in the LRR cohort (7.8\%). One patient in the LVR cohort suffered from extensive pelvic adhesions, requiring adhesiolysis, which was complicated by serosal injury of the small bowel. A conversion to laparotomy was needed in order to perform an adequate repair of this lesion.

Due to mobilization of the splenic flexure of the colon during LRR, a splenic tear occurred in one patient. Hemostasis was adequately achieved with Surgicel (Ethicon, Johnson\&Johnson, Hamburg, Germany). Total was blood loss was $<500 \mathrm{ml}$. The second intraoperative complication in this group was a technical failure of the stapling device. Resection of the incomplete staple line was necessary and a complete new anastomosis was created. No anastomotic leakage occurred.

Three postoperative complications (7.5\%, all in different patients) occurred in the LVR group compared to 9 complications (32.1\%, in 8 different patients) $(p<0.05)$ in the LRR group. An overview of all postoperative complications according to the Clavien-Dindo classification can be found in table 2.

Table 2 Overview of all postoperative complications according to the Clavien-Dindo (CD) classification. Except for 2 complications they all occurred in different patients. The * indicates the two complications that developed in the same patient after LRR.

\begin{tabular}{|c|c|c|c|c|c|}
\hline \multicolumn{3}{|c|}{$\begin{array}{l}\text { Laparoscopic resection rectopexy: } \\
\text { Postoperative complications \& management }\end{array}$} & \multicolumn{3}{|c|}{$\begin{array}{l}\text { Laparoscopic ventral rectopexy: } \\
\text { Postoperative complications \& management }\end{array}$} \\
\hline & $N=(\%)$ & $\begin{array}{l}\text { Clavien- } \\
\text { Dindo } \\
\text { Class. }\end{array}$ & & $N=(\%)$ & $\begin{array}{l}\text { Clavien- } \\
\text { Dindo } \\
\text { Class. }\end{array}$ \\
\hline $\begin{array}{l}\text { Prolonged ileus treated } \\
\text { consevatively }\end{array}$ & 4 (14.3\%) & Grade I & $\begin{array}{l}\text { Prolonged ileus treated } \\
\text { conservatively }\end{array}$ & $1(2.5 \%)$ & Grade I \\
\hline $\begin{array}{l}\text { Wound infection treated } \\
\text { with opening of the wound }\end{array}$ & $2(7.1 \%)$ & Grade I & $\begin{array}{l}\text { Myocardial ischemia not } \\
\text { requiring intervention }\end{array}$ & $2(5.0 \%)$ & Grade IV \\
\hline $\begin{array}{l}\text { Pneumonia treated with } \\
\text { IV antibiotics }\end{array}$ & $1(3.6 \%)$ & Grade II & & & \\
\hline $\begin{array}{l}\text { Pulmonary embolism } \\
\text { treated with anticoagu- } \\
\text { lant therapy* }\end{array}$ & $1(3.6 \%)$ & Grade II & & & \\
\hline $\begin{array}{l}\text { Intra- abdominal fluid } \\
\text { collection drained by } \\
\text { interventional radiology* }\end{array}$ & $1(3.6 \%)$ & Grade IIIa & & & \\
\hline $\begin{array}{l}\text { Total number of } \\
\text { complications }\end{array}$ & $9(32.1 \%)$ & & $\begin{array}{l}\text { Total number of } \\
\text { complications }\end{array}$ & $3(7.5 \%)$ & \\
\hline
\end{tabular}




\section{Functional results}

Obstructed defecation syndrome and constipation

Before surgery, 16 patients (57\%) reported symptoms of constipation or ODS in the LRR group. A comparable incidence of these symptoms was found pre-operatively in the LVR group (22 patients, 55\%). During the follow-up period, symptoms resolved in the majority of patients in both groups: 6 patients $(21.4 \%)$ had complaints of constipation/ODS in the LRR cohort versus $9(23 \%)$ in the LVR cohort. In both groups new onset constipation was seen in one patient. The postoperative decrease of constipation/ODS was significant for both groups compared to the preoperative incidence.

When comparing the efficacy of the two techniques relative to pre- and post-operative functional results, no significant differences between the two study groups were observed. An overview of functional results can be found in tables $\mathbf{3 a}$ (incontinence) and $\mathbf{3 b}$ (constipation).

Table 3a Incontinence

\begin{tabular}{lccc}
\hline & LRR & LVR & P-value \\
\hline Preoperative incontinence & $17(61 \%)$ & $22(55 \%)$ & 0.73 \\
Postoperative incontinence & $1(4 \%)$ & $6(15 \%)$ & 0.50 \\
P-value & $<0.01$ & $<0.01$ & \\
\hline
\end{tabular}

$\mathrm{LRR}=\mathrm{Laparoscopic}$ resection rectopexy; LVR= Laparoscopic ventral rectopexy.

Table 3b Constipation

\begin{tabular}{lccc}
\hline & LRR & LVR & P-value \\
\hline Preoperative constipation & $16(57 \%)$ & $22(55 \%)$ & 0.48 \\
Postoperative constipation & $6(21 \%)$ & $9(23 \%)$ & 1.00 \\
P-value & $<0.01$ & $<0.01$ & \\
\hline
\end{tabular}

LRR= Laparoscopic resection rectopexy; LVR= Laparoscopic ventral rectopexy.

\section{Incontinence}

During preoperative evaluation, symptoms of incontinence were reported by 17 patients (61\%) in the LRR group and by 22 patients (55\%) in the LVR cohort. During follow-up, a significant decrease in incontinence was observed for both groups: 1 patient in the LRR cohort (3.6\%) versus 6 patients in the LVR group $(15 \%)$ had persistent incontinence. Direct comparison of the two surgical techniques showed a trend towards significance $(p=0.09)$. 


\section{Recurrences}

There were no recurrences or reoperations because of pelvic floor prolapse in the two groups.

\section{Discussion}

Although rectal prolapse is a common problem, the optimal surgical treatment for it has not been determined yet. As prospective studies between LRR and LVR are still in the recruitment phase and evidence in the literature is lacking, the aim of this current study was to retrospectively compare these two most frequently used surgical strategies for the treatment of patients with RP. It was found that both treatment strategies offer a significant reduction of symptoms of incontinence and constipation when compared to the preoperative incidence. This finding is consistent with the current literature: both LVR and LRR seem to be successful in reducing symptoms of RP in series describing either one of these techniques [13-16].

When the results of the two techniques are directly compared, almost identical percentages of pre- and postoperative symptoms of constipation were found in the two groups, indicating that no significant difference between these two techniques existed. Preoperative incontinence was observed in similar incidences in the two groups, however it persisted more frequently after surgery in the LVR group ( $15 \%$ vs. $4 \%$ ). The origin of this difference is not clearly understood by the authors. Possibly, the significantly older age of the LVR cohort might cause the higher rate of incontinence in this cohort, as more factors causing the involuntary loss of feces might be present in these patients. Improved continence and constipation in patients after LVR and LVR seems to be caused by restored anatomy, probably resulting in a better function of the rectum and better sensitivity for feces in the rectum. Therefore, another possible explanation for this difference is that LVR causes less restoration of physiological rectal function compared to LRR. Although this difference in postoperative incontinence was not significant in this relatively small study, it is something that needs to be addressed in larger, prospective, studies.

In the LRR cohort, a significantly higher incidence of complications was found compared to the LVR group. This finding was primarily caused by low-grade (I-II) complications such as successfully conservatively managed prolonged ileus and wound infections. The number of high-grade complications was not significantly different between the cohorts and consisted of myocardial ischemia in two elderly patients with a history of heart disease in the LVR group. In the LRR group, one patient suffered from an intra-abdominal fluid collection, which was drained radiologically. Although a rectosigmoid resection with creation of an anastomosis is performed during LRR, no anastomotic leakage oc- 
curred. Previous studies describing LRR also found low percentages of anastomotic leakage, or as in our study no cases of leakage at all. $[5,13]$ The number of minor and major complications after LRR in literature seem to be comparable to the current study, being between 13 and 30\% for minor complications and around $5 \%$ for major complications $[13,15,17,18]$. In general, the complication rate after LVR is slightly lower in the literature: several series of patients after LVR have been published in recent years, all showing minor complication rates below $17 \%[6,10,16]$. This finding was also noted in the current study. Major complications, for example mesh infection and mesh erosions after LVR, seem to be rare, although some cases of lumbar discitis at the proximal fixation point of the mesh have been described [19].

Recently, the results of the PROSPER trial (the largest randomized trial to date comparing different surgical techniques for the treatment of rectal prolapse) were published. This study compared abdominal vs. perineal approaches including laparoscopic rectopexy with and without resection. No significant differences were found in functional outcomes and recurrences between the different techniques. LVR was unfortunately not studied in this high quality project as it was initiated in the early 2000's before LVR was widely used. Prospective, comparative studies between LVR and LRR are therefore still needed [20].

The present study has several limitations. Due to the retrospective nature of this study, the scoring of preoperative and postoperative symptoms was not systematically recorded using validated questionnaires and therefore had to be determined using patients' charts. Symptoms were retrospectively scored as 'present' or 'absent' by the same reviewer in the two centers, using the Rome II criteria, instead of being prospectively quantified using validated scoring systems. Also, underreporting of minor complications is possible. Patients were discharged after a mean of less than 6 days and, for example, some complications may not have been noted in an outpatient setting (or at the general practitioner) . Furthermore, some selection bias exists due to the design of this international, retrospective cohort study. For example, the American group (LRR) was significantly younger then the European cohort, probably because traditionally, elderly patients underwent an Altermeier's perineal rectosigmoidectomy at Cleveland Clinic Florida, while basically all adult patients with an external RP underwent an LVR in the Netherlands. This policy for octogenarians was applied after this procedure proved to be safe and effective in the elderly, both in local experience as in the literature [21].

Other non-measurable differences between the two groups in this study will certainly exist. These are discrepancies caused by the international, multi-center study design of this study. For example, cultural and educational differences will cause some variations in daily and medical practice between the two hos- 
pitals. As uniformity was found between the two centers concerning the main endpoints of this study (discharge criteria were identical, definitions of incontinence and constipation were comparable, as was the registration of complications), it proved feasible to compare these two cohorts.

\section{CONCLUSIONS}

Both LRR and LVR seem safe and suitable treatment strategies for external RP. Although there were more complications in the LRR cohort, this technique might offer better improvement of incontinence. Prospective studies are awaited to further determine differences in functional results and complications between these two techniques. 


\section{References}

1. Matzel KE, Heuer S, Zhang W (2008) Rectalprolapse:Abdominal or local approach. Chirurg 79:444-451

2. Madiba TE, Baig MK, Wexner SD (2005) Surgical management of rectal prolapse. Arch Surg 140:63-73

3. Wu JS (2009) Rectal prolapse: a historical perspective. Curr Probl Surg 46:602-716

4. Kuijpers HC (1992) Treatment of complete rectal prolapse: to narrow, to wrap, to suspend, to fix, to encircle, to plicate or to resect? World J Surg16:826-830

5. Laubert T, Bader FG, Kleemann M et al (2012) Outcome analysis of elderly patients undergoing laparoscopic resection rectopexy for rectal prolapse. Int J Colorectal Dis 27:789-795

6. Wijffels N, Cunningham C, Dixon A, Greenslade G, Lindsey I (2001) Laparoscopic ventral rectopexy for external rectal prolapse is safe and effective in the elderly. Does this make perineal procedures obsolete? Colorectal Dis 13:561-566

7. Clark CE III, Jupiter DC, Thomas JS, Papaconstantinou HT (2012) Rectal prolapse in the elderly: trends in surgical management and outcomes from the american college of surgeons national surgical quality improvement program database. J Am Coll Surg 215:709-714

8. Magruder JT, Efron JE, Wick EC, Gearhart SL (2013) Laparoscopic rectopexy for rectal prolapse to reduce surgical-site infections and length of stay. World J Surg 37:1110-1114

9. Cadeddu F., Sileri P., Grande M., De Luca E., Franceschilli L., Milito G. (2013) Focus on abdominal rectopexy for full-thickness rectal prolapse: meta-analysis of literature. Tech Coloproctol 16:37-53

10. Formijne Jonkers HA, Draaisma WA, Wexner SD et al (2013) Evaluation and surgical treatment of rectal prolapse: An international survey. Colorectal Dis15:115-119

11. D’Hoore A, Penninckx F (2006) Laparoscopic ventral recto(colpo)pexy for rectal prolapse: surgical technique and outcome for 109 patients. Surg Endosc 20:1919-1923

12. Dindo D, Demartines N, Clavien PA (2004) Classification of surgical complications: a new proposal with evaluation in a cohort of 6336 patients and results of a survey. Ann Surg 240:205-213

13. Ashari LH, Lumley JW, Stevenson AR, Stitz RW (2005) Laparoscopically-assisted resection rectopexy for rectal prolapse: ten years' experience. Dis Colon Rectum 48:982-987

14. D’Hoore A, Cadoni R, Penninckx F (2004) Long-term outcome of laparoscopic ventral rectopexy for total rectal prolapse. Br J Surg 91:1500-1505

15. Stevenson AR, Stitz RW, Lumley JW (1998) Laparoscopic-assisted resection-rectopexy for rectal prolapse: early and medium follow-up. Dis Colon Rectum 41:46-54

16. Maggiori L., Bretagnol F., Ferron M., Panis Y. (2013) Laparoscopic ventral rectopexy: a prospective long-term evaluation of functional results and quality of life. Tech Coloproctol 17:431-436

17. Boons P, Collinson R, Cunningham C, Lindsey I (2010) Laparoscopic ventral rectopexy for external rectal prolapse improves constipation and avoids de novo constipation. Colorectal Dis 12:526-532 
18. Laubert T, Kleemann M, Schorcht A et al (2010) Laparoscopic resection rectopexy for rectal prolapse: a single-center study during 16 years. Surg Endosc 24:2401-2406

19. Draaisma WA, van Eijck MM, Vos J, Consten EC (2011) Lumbar discitis after laparoscopic ventral rectopexy for rectal prolapse. Int J Colorectal Dis 26:255-256

20. Senapati A, Gray RG, Middleton LJ et al (2013) PROSPER: a randomised comparison of surgical treatments for rectal prolapse. Colorectal Dis 15:858-868

21. Wijffels N, Cunningham C, Dixon A et al (2011) Laparoscopic ventral rectopexy for external rectal prolapse is safe and effective in the elderly. Does this make perineal procedures obsolete? Colorectal Dis 13:561-566 


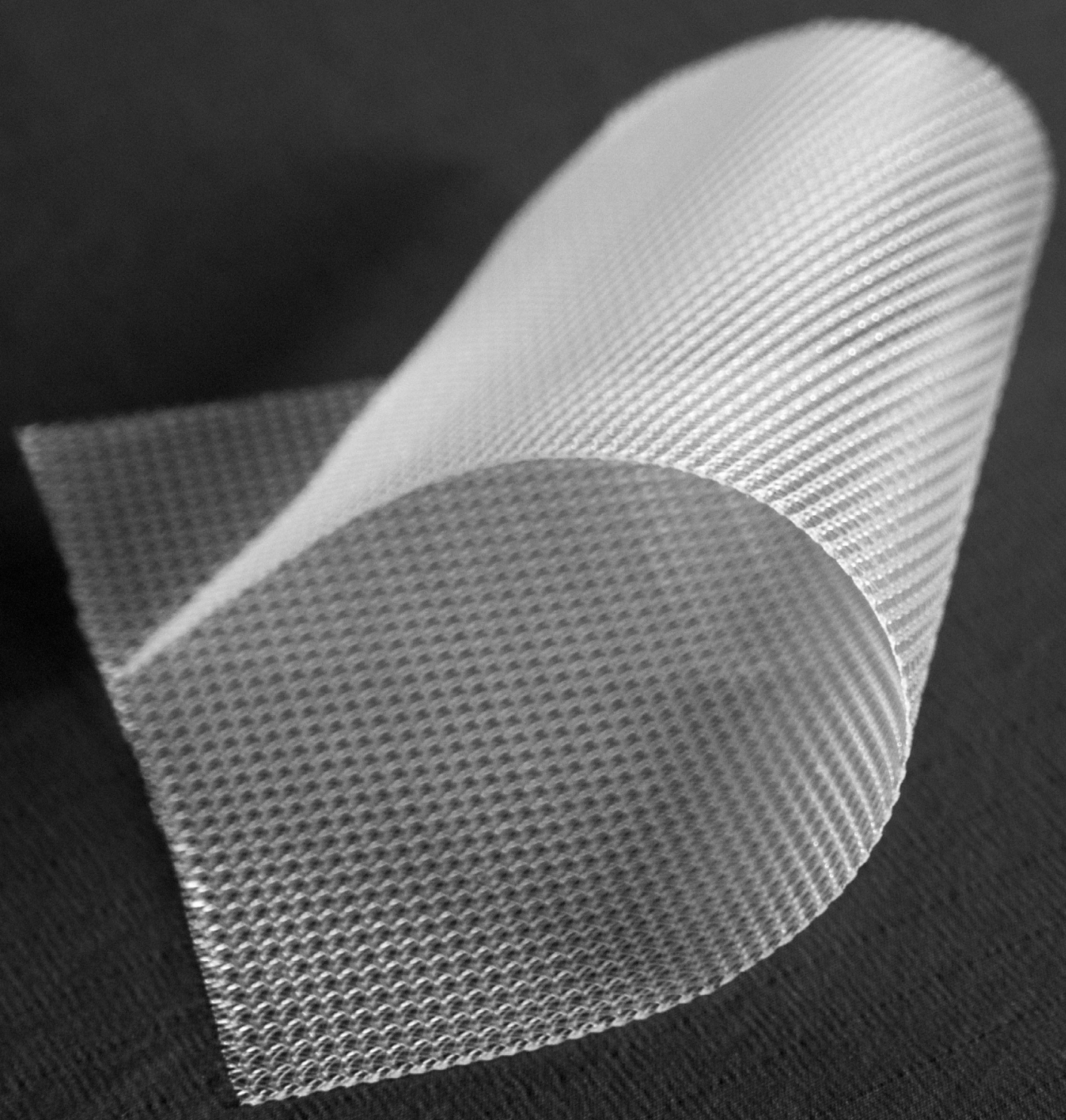




\title{
Chapter 6
}

\author{
High-grade hemorrhoids \\ requiring surgical treatment \\ are common after \\ laparoscopic ventral rectopexy
}


Aim

This retrospective study aimed to determine the incidence of grade III and IV hemorrhoids requiring surgery in patients who received Laparoscopic Ventral Rectopexy (LVR) in the past and describe complications of the hemorrhoidectomy.

\section{Methods}

All patients treated with LVR between March 2004 and May 2013 were identified and enrolled in a retrospective database. The incidence of surgical treatment because of grade III / IV hemorrhoids was registered. All relevant patient and follow-up characteristics where gathered.

\section{Results}

A total of 421 patients were identified. Sixty-five of these patients (15.4\%, 4 males, 61 females) developed symptomatic grade III / IV hemorrhoids requiring a hemorrhoidectomy during follow-up. Mean age was 60.4 years $(27-84)$. The mean duration between
LVR and hemorrhoidectomy was 9.04 months (2.53 - 45.31, SD 8.68). Twelve of the 65 patients (18.5\%) eventually developed a recurrence of a rectal prolapse. Re-do surgery because of recurrent hemorrhoids was needed in 15 patients $(23.1 \%)$ after a mean of 9.0 months after the initial hemorrhoidectomy. The complication rate was $13.8 \%(n=9)$ and $20.0 \%(n=3$, redo). One intra-operative complication occurred (6.7\%).

\section{Conclusions}

The incidence (residual) grade III / IV hemorrhoids after rectopexy is high. For these patients, hemorrhoidectomy is an effective and safe operation although the recurrence rate is higher compared to patients with no previous prolapse surgery. Thereby it appeared that hemorrhoids after LVR are associated with a higher recurrence rate of rectal prolapse as compared to recent literature. 


\section{INTRODUCTION}

Pelvic floor disorders is a term that describes a variety of clinical conditions, including urinary and fecal incontinence, pelvic organ prolapse, obstructed defecation and chronic pelvic pain. In the western world, it is a common problem affecting more than $40 \%$ of the middle-aged and older women, with a lifetime risk of undergoing surgery of $10-20 \%[1,2]$. The rectum is often involved in this multi-organ problem [3]. Various conditions including rectoceles, internal and external rectal prolapse may cause fecal incontinence and the obstructed defecation syndrome (ODS) [4].

Laparoscopic ventral rectopexy is increasingly applied for the treatment of external rectal prolapse, internal rectal prolapse and large symptomatic rectoceles. This technique proved to be safe and effective in terms of functional results in large cohorts of patients [5-9]. However the incidence of large hemorrhoids seems high after LVR and surgical correction is common. These hemorrhoids are probably due to a residual mucosal prolapse after surgery. This issue has not been debated in literature before. The purpose of this study was to determine the incidence of high-grade hemorrhoids requiring surgical treatment after rectopexy in our large cohort of patients.

\section{PATIENTS AND METHODS}

\section{Study design}

This retrospective cohort study was performed in a large teaching hospital in the Netherlands. All consecutive patients who underwent LVR for various indications between March 2004 and May 2013 were analyzed. Individuals with (residual) grade III and IV hemorrhoids requiring surgical treatment were enrolled in a registry database. Relevant patient data and demographics including medical history, surgical details and follow-up information including recurrence and complications were recorded.

\section{Patients and evaluation}

Regular post-operative care and follow-up after LVR was carried out according to a standardized protocol and performed at 6 weeks after surgery. All patients started with a laxative (Macrogol 3350/eletrolytes, Movicolon ${ }^{\circledR}$, Norgine Limited, Mid Glamorgan, U.K.) directly after LVR procedure. During outpatient visit an assessment of recurrence, complications, continence and constipation using the anamnesis, medical history and physical examination was performed. In case of complaints, the existence of hemorrhoids were examined in standing and in lithomy position using leg supports, both in rest and during straining. Proctoscopy was performed additionally. Patients with grade II and III hemorrhoids were treated with rubber band ligation (RBL) first. Persisting symptomatic grade III/IV hemorrhoids were considered an indication for surgery. 


\section{Surgical technique}

Either a Procedure for Prolapse and Hemorrhoids (PPH) or a traditional excisional hemorrhoidectomy was executed. Surgery was performed by (or under direct supervision of) an experienced pelvic floor surgeon. Preoperative antibiotics were not routinely administered. All operations were performed under general or spinal anesthesia. The patients were placed in the lithomy position using adaptive leg supports with swing stirrups. In stapled hemorrhoidectomy either the PPH 03 stapler produced by Ethicon (EndoSurgery, Cincinnati, Ohio, USA) or the EEA HEM3335 made by Covidien (Mansfield, Massachusetts, USA) was used. The stapled procedure has been previously standardized and was performed according to the technique described by Singer [10]. Excisional hemorrhoidectomy was performed according to standard protocol [11].

\section{Outcome parameters}

The main outcome parameters of this study were incidence of patients receiving a surgical hemorrhoid treatment after a previous LVR and accompanying intra and postoperative complications. Postoperative complications during hospitalization were assessed using the Clavien-Dindo Classification (CD) $[12,13]$.

\section{Statistical Analysis}

Statistical Package for the Social Science, version 20.0 (SPSS Inc., Chicago, IL, USA) was used for statistical analysis. Data are presented as percentage, mean \pm S.D, median and range. For the descriptive analyses, McNemar tests were used to evaluate differences in percentages. $\mathrm{P}<0.05$ was considered statistically significant.

\section{RESULTS}

\section{Patient characteristics}

A total of 421 patients (16 males; 405 females) underwent a LVR for external rectal prolapse, internal rectal prolapse or large symptomatic rectocele (with or without enterocele). Hemorrhoidectomy or a PPH because of grade III / IV hemorrhoids had been performed in 20 patients before LVR .

During follow-up, 85 patients $(20.2 \%)$ required additional treatment for hemorrhoids.

Twenty patients (4.8\%) were treated sufficiently by RBL. 65 patients (15.4\%) 4 males, 61 females) received surgical treatment for symptomatic grade III / IV hemorrhoids. 63 patients underwent a stapled hemorrhoidectomy and two a traditional excisional hemorrhoidectomy. Seventeen out of these 65 patients (26.2\%) had RBL first, without sufficient result.. The mean duration between LVR and hemorrhoid surgery was 9.04 months (2.53 - 45.31. SD 8.68). Mean age 
at surgery was 60.4 years $(27-84)$. In one patient PPH was combined with a posterior colporrhaphia because of an anterior rectocele. General patient characteristics are presented in Table $\mathbf{1}$.

Table 1. Patient characteristics and medical history.

\begin{tabular}{lccc}
\hline Patient characteristics & LVR & PPH & \\
\hline Gender & Male & $16(3,8 \%)$ & $4(6,2 \%)$ \\
& female & $405(96,2 \%)$ & $61(93,8 \%)$ \\
Age & Years & $61,8(22-95)$ & $60,4(27-84)$ \\
& & SD 13,14 & SD 12.95 \\
Primair of re-do LVR & Primair & $384(91,2 \%)$ & $57(87,7 \%)$ \\
& Re-do & $37(8,8 \%)$ & $8(12,3 \%)$ \\
\hline History & & & \\
\hline Para [episiotomy] & $2,4(0-10)[37$ in 25 & $2,6(0-5)[12$ in 7 & \\
& pt] SD 1,43 & pt] SD 1,49 & \\
Hysterectomy & $140(33,3 \%)$ & $47(72,3 \%)$ & \\
Cystopexy & $39(9,3 \%)$ & $4(6,2 \%)$ & \\
Colporrhaphia anterior & $56(13,3 \%)$ & $13(20,0 \%)$ & \\
Sfincter operation & $6(1,4 \%)$ & 0 & \\
Other abdominal surgery & $138(32,8 \%)$ & $23(35,4 \%)$ & \\
Rubber band ligation after LVR & $39[13](9,3 \%)$ & $17[4](26,2 \%)$ & \\
[second session] & $20(4,8 \%)$ & $2(3,1 \%)$ & \\
Pre-PPH (before initial LVR) & & & \\
\hline
\end{tabular}

\section{Follow-up}

The mean follow-up after hemorrhoidectomy was 14.5 months (0.76- 68.34. SD 16.68). In the postoperative course 15 patients (23.1\%) needed re-do surgery ( $n=13 \mathrm{PPH}$ ) for persistent or recurrent grade III / IV hemorrhoids after a mean of 9.0 months $(1.5-40.5$. SD 9.47). One patient received an excisional hemorrhoidectomy after two times a PPH in a period of 8.9 months. Twelve of the 65 patients (18.5\%) who underwent hemorrhoidectomy eventually developed a recurrence of a rectal prolapse for which a re-do rectopexy was indicated. of those, 9 patients underwent a re-do LVR. One of these patients underwent a sutured transanal mucosectomy and plication before and a sigmoid resection because of a dolichosigmoïd with intussusception of the rectum after the redo rectopexy. Another PPH was performed in one patient 9 months after re-do 
LVR. The remaining 3 patients with a recurrence choose not to be operated. Of the patients developing a recurrence five patients received a dynamic MRI after LVR and before the PPH. Mean duration between the initial LVR and eventually developing a recurrence was 26.79 months (10.94 - 53.78. SD 14.64).

Due to persisting fecal incontinence without curative surgical options, a stoma was created in three patients (1 loop ileostomy, 1 sigmoidstomy and 1 loop transversostomy). See Figure $\mathbf{1}$ for the complete flowchart.

\section{Surgical details hemorrhoidectomy}

Of the initial $63 \mathrm{PPHs}$, thirteen (20.6\%) hemorrhages at the suture line were oversewn after firing of the stapler and in $6(9.5 \%)$ patients additional mucosa was resected because of an incomplete staple line. Three patients $(23.1 \%)$ who underwent a re-PPH needed stitches for hemostasis and 4 (30.8\%) needed resection of mucosa after stapling.

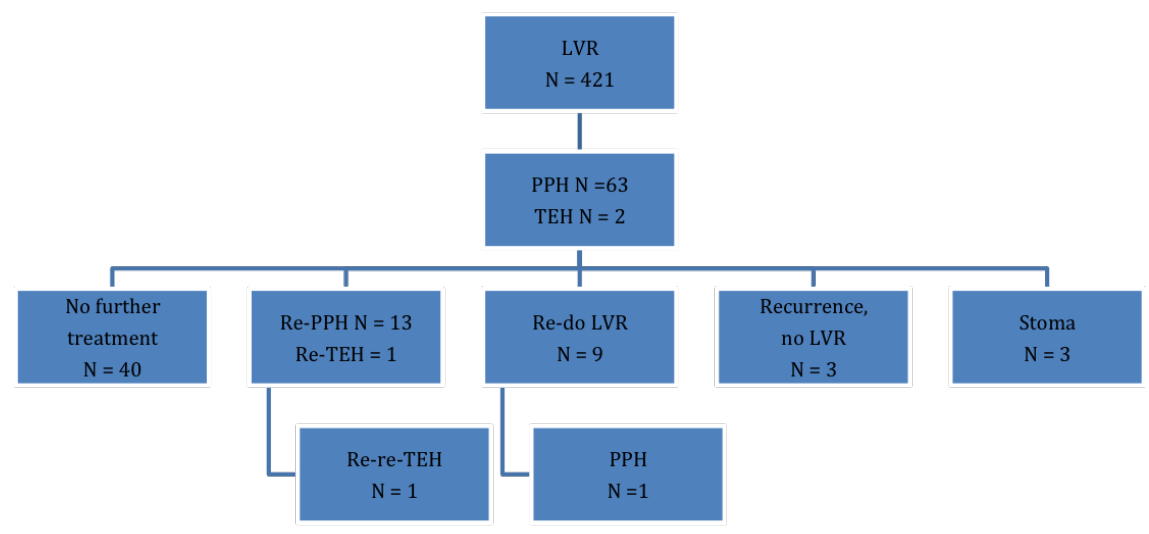

Figure 1. Flowchart.

\section{Early and late complications}

Hemorrhoidectomy: complications during surgery and follow-up

No intra-operative complications occurred. The complication rate was $13.8 \%$ $(n=9)$ and all of these patients received a PPH. Two patients developed urinary retention for which a catheter was placed for seven and twenty days. Within six days after discharge 4 patients presented themselves at the emergency department of which one patient with an urinary tract infection, one patient with complaints of nausea probably due to the general anesthesia and one patient with a subsequent bleeding which was treated expectative. The fourth patient 
complained of warm and strange sensation under the level where the epidural was given. In agreement with the anesthesiologist the complaints were interpreted as non-specific and the patient was sent home.

The last three complications were seen at the outpatient clinic. One patient suffered from fecal incontinence and intra-anal pain, which could be explained because of persisting staples near the dentate line perforating the mucosa. Surgical removal of these staples was necessary under spinal anesthesia. One patient had persisting blood loss per anum and grade III hemorrhoids for which a re-PPH was executed.

One patient developed dyspareunia. Mean time between surgery date and the complication was 1.25 months ( $0-5.65$. SD 1.98).

Re-do hemorrhoidectomy: complications during surgery and follow-up

One intra-operative complication took place during re-PPH. In this case the stapler was positioned above the staple line of the previous PPH. Firing of the stapler caused a perforation in the ventral rectal wall at the location of the prolene ventral rectopexy mesh. During inspection, no perforation of the posterior vaginal wall was observed and the rectal wall was transanally closed in layers. Postoperative, amoxicillin/clavulanic acid (Augmentin) IV $1200 \mathrm{mg}$ q8h was administered for three days. At discharge Augmentin was switched to $625 \mathrm{mg}$ q $8 \mathrm{~h}$ orally for 7 additional days. No mesh infection or further complications occurred in this case except for dyspareunia and a tight feeling around the vagina. These complaints resolved after an operation where a piece of the mesh near the vagina and two sutures at the location of the uterosacral ligament were removed. This postoperative complication was one of the three in total $(n=3,20.0 \%)$. One patients suffered from postoperative hypokalemia for which one-off potassium $30 \mathrm{ml}$ per os was given. At discharge slow-k tablets $600 \mathrm{mg} 98 \mathrm{~h}$ were started. The third patient was postoperatively administered to the intensive care for observation because of OSAS complaints. One day later the patient moved to the ward with no further complications.

There was no surgical related mortality during surgery and follow-up. An overview of all post-operative complications according to the Clavien-Dindo [12,13] classification can be found in Table 2. 
Table 2. Complications classified according to the Clavien-Dindo classification 10, 11.

\begin{tabular}{|c|c|c|c|}
\hline Procedure & Post-operative complications \& management & $n=(\%)$ & CD Class. \\
\hline \multirow[t]{9}{*}{ PPH } & $\begin{array}{l}\text { Fecal incontinence and intra-anal pain: surgi- } \\
\text { cal removal of staples }\end{array}$ & $1(1,5 \%)$ & Grade IIIb \\
\hline & Persisting blood loss per anum: re-PPH & $1(1,5 \%)$ & Grade IIIb \\
\hline & Dyspareunia: re-PPH & $1(1,5 \%)$ & Grade IIIb \\
\hline & Urinary retention: catheter, 7 and 20 days & $2(3,1 \%)$ & Grade II \\
\hline & Urinary tract infection: antibiotics & $1(1,5 \%)$ & Grade II \\
\hline & $\begin{array}{l}\text { Subsequent bleeding: expectant manage- } \\
\text { ment }\end{array}$ & $1(1,5 \%)$ & Grade I \\
\hline & Nausea: metoclopramide & $1(1,5 \%)$ & Grade I \\
\hline & $\begin{array}{l}\text { Warm/strange sensation under epidural level: } \\
\text { expectant management }\end{array}$ & $1(1,5 \%)$ & Grade I \\
\hline & Total & $9(13,8 \%)$ & \\
\hline \multirow[t]{4}{*}{ Re-PPH } & $\begin{array}{l}\text { Dyspareunia: surgical removal piece of mesh } \\
\text { and two sutures. }\end{array}$ & $1(6,7 \%)$ & Grade IIIb \\
\hline & Hypokalemia: electrolytes & $1(6,7 \%)$ & Grade I \\
\hline & OSAS complaints: 1 night ICU for observation & $1(6,7 \%)$ & Grade I \\
\hline & Total & $1(20,0 \%)$ & \\
\hline
\end{tabular}

CD: Clavien-Dindo classification.

$\mathrm{PPH}$ : procedure for prolapse and hemorrhoids

TEH: traditional excisional hemorrhoidectomy

\section{Discussion}

High-grade hemorrhoids are common in the adult general population with an incidence of $3.5 \%$. The majority of these patients is symptomatic [14]. For grade III and IV hemorrhoids, surgery may be indicated. The two most used techniques are the traditional excisional hemorrhoidectomy and the $\mathrm{PPH}$, also called a stapled hemorrhoidopexy [15]. The technique of choice for symptomatic patients with hemorrhoids grade III and IV in our institution is the PPH. Since first described by Longo [16] in 1998, PPH is increasingly performed. Multiple randomized controlled trials reported less pain and a shorter convalescence time than traditional excisional hemorrhoidectomy [17]. The aim of this study was to determine the incidence of (residual) hemorrhoids grade III and IV in patients after previous LVR. To our knowledge there is no earlier documentation on this specific problem. With $15.4 \%$ of the patients requiring surgery, a high incidence was found. Before LVR 34 patients (52.3\%) of this series had ODS complaints. Most of them had a long history of straining and incomplete evacuation. Laborious defecation has become a second nature for these patients. 
The high incidence of grade III / IV hemorrhoids could be explained because of persistent straining after the LVR. Furthermore, it is known that persisting constipation (7.1 to $19.0 \%$ ) is common after LVR [5,8].

Due to this postoperative laxatives are prescribed in all patients. This may also explain the very high recurrence rate of grade III / IV hemorrhoids for which re-do surgery was indicated $(n=15,23.1 \%)$. In the literature several randomized controlled trials showed recurrences from 0 to 5.0\% [18-22]. The relatively low incidence of patients who received a pre-hemorrhoidectomy RBL (26.2\%) may also be an explanation of higher recurrence of residual grade III / IV hemorrhoids. Namely, recent literature investigating predictors of recurrence after hemorrhoidectomy showed that the absence of previous RBL was associated with higher recurrence [23]. The incidence of recurrence of rectal prolapse in this specific group of patients $(n=12,18.5 \%)$ is also high compared to recurrence rates after LVR in recent studies, varying from 0 to $5.4 \%$ [5,6,8,24-27]. This could indicate that patients with hemorrhoids after rectopexy are susceptible to develop a recurrence after LVR. This cohort seems to contain a population segment of patients with persisting symptoms who are difficult to treat. Both high recurrence rates of rectal prolapse and grade III / IV hemorrhoids are indicative. The three patients receiving a stoma due to persistent fecal incontinence, and therefore not counted as recurrences, support the assertion that this specific group is difficult to treat.

A high percentage of the patients suffer from combined problems and it appears that this interferes with the outcome as the results of either the initial LVR and the postoperative PPH are moderate with both high recurrences. It seems that the complexity of the patients is a significant contributing factor on the outcome of a procedure in this study. The occurrence of grade III / IV hemorrhoids after LVR may be a sign of a patient with a complex pelvic floor disorder possibly not well responding to the standard therapy. Consequently, surgeons could consider additional radiological imaging when a patient presents itself with grade III and IV hemorrhoids in the postoperative follow-up after LVR. In addition it is noticeable that the PPH group includes more patients with a hysterectomy $(72.3 \%$ vs. $33.3 \%)$ re-do of the initial LVR (12.3\% vs. $8.8 \%)$ and episiotomy $(10.7 \%$ vs. $5.9 \%)$ in the past compared to the whole LVR group.

LVR is being performed since 2004 in our hospital. Analysis shows that the patients with a post LVR hemorrhoidectomy are quite similar distributed over the years. This indicates that there is probably no learning curve problem, or sign of insufficient repair.

In our center, PPH was predominantly done and complications only occurred after this procedure. A large systematic review showed an overall complication rate of $20.2 \%$ after PPH but the severity of the individual complications was not split out [28]. Another multicenter study with more than 500 patients in- 
cluded reported $19.2 \%$ complications [29]. Our complication rate for the initial hemorrhoidectomy was lower with $13.8 \%$ but for the re-do a similar percentage was found (20.0\%). Four of the complications needed re-intervention (CD grade $\mathrm{IIla} / \mathrm{b}$ ). Literature shows that 5 to $11 \%$ needs a re-intervention for complication at one year follow-up [30]. Postoperative fecal incontinence and intra anal pain are both associated with a low placed staple line [31,32]. Postoperative fecal incontinence of 3.2 to $17 \%$ after PPH for grade II-IV hemorrhoids has been described $[30,31,33]$. Another common complication is rectal bleeding. After PPH from 1.8 to $11.2 \%$ rectal bleeding within 24 hours [11,29,34] and 1.7 to $9.8 \%$ after discharge is seen $[11,28,34,35]$. A systematic review showed a incidence of $3.7 \%$ of patients with bleeding requiring surgical intervention or re-admission [36]. Urinary retention is reported in $0.9-13.8 \%$ of the cases $[11,20,27,29,34,36]$. In general, rectal perforation due to PPH is rare. A one-year German survey of $4635 \mathrm{PPH}$ by Herold et al. reported a total of 3 rectal perforations [37]. In our study however the PPH stapler perforated the rectal wall in one case and the staples were placed through the ventral rectopexy mesh. This complications was successfully treated by removing the staples out of the mesh, closure of the rectal wall in layers and administering IV antibiotics during several days. Surgeons considering a PPH after LVR should be very cautious and aware of the location of the mesh at all times to avoid similar complications.

In conclusion, patients after LVR procedure have a high incidence of (residual) grade III / IV hemorrhoids. For these patients, hemorrhoidectomy (traditional or $\mathrm{PPH}$ ) is a safe and effective operation although the recurrence rate of mucosal prolapse is higher than in patients with no previous prolapse surgery. Thereby the recurrence of rectal prolapse requiring a re-do LVR is strikingly high possibly indicating this study contains a population segment of patients with pelvic floor disorders who are complex. 


\section{REFERENCES}

1. de Boer TA, Slieker-Ten Hove MC, Burger CW, Kluivers KB, Vierhout ME. The prevalence and factors associated with previous surgery for pelvic organ prolapse and/or urinary incontinence in a crosssectional study in The Netherlands. Eur J Obstet Gynecol Reprod Biol. 2011;158:343-349.

2. Sung VW, Hampton BS. Epidemiology of pelvic floor dysfunction. Obstet Gynecol Clin North Am. 2009;36:421-443.

3. Elneil S. Complex pelvic floor failure and associated problems. Best Pract Res Clin Gastroenterol. 2009;23:555-573.

4. Beck DE, Allen NL. Rectocele. Clin Colon Rectal Surg. 2010;23:90-98.

5. D'Hoore A, Cadoni R, Penninckx F. Long-term outcome of laparoscopic ventral rectopexy for total rectal prolapse. Br J Surg. 2004;91:1500-1505.

6. D'Hoore A, Penninckx F. Laparoscopic ventral recto(colpo)pexy for rectal prolapse: surgical technique and outcome for 109 patients. Surg Endosc. 2006;20:1919-1923.

7. Formijne Jonkers HA, Draaisma WA, Wexner SD, et al. Evaluation and surgical treatment of rectal prolapse: an international survey. Colorectal Dis. 2013;15:115-119.

8. Formijne Jonkers HA, Poierrie N, Draaisma WA, Broeders IA, Consten EC. Laparoscopic ventral rectopexy for rectal prolapse and symptomatic rectocele: an analysis of 245 consecutive patients. Colorectal Dis. 2013;15:695-699.

9. Wong MT, Abet E, Rigaud J, Frampas E, Lehur PA, Meurette G. Minimally invasive ventral mesh rectopexy for complex rectocoele:impact on anorectal and sexual function.Colorectal Dis. 201113:e320

10. Singer MA, Cintron JR, Fleshman JW, et al. Early experience with stapled hemorrhoidectomy in the United States. Dis Colon Rectum. 2002;45:360-7; discussion 367-9.

11. Kim JS, Vashist YK, Thieltges S, et al. Stapled hemorrhoidopexy versus milligan-morgan hemorrhoidectomy in circumferential third-degree hemorrhoids: long-term results of a randomized controlled trial. J Gastrointest Surg. 2013;17:1292-1298.

12. Clavien PA, Barkun J, de Oliveira ML, et al. The Clavien-Dindo classification of surgical complications: five-year experience. Ann Surg. 2009;250:187-196.

13. Dindo D, Demartines N, Clavien PA. Classification of surgical complications: a new proposal with evaluation in a cohort of 6336 patients and results of a survey. Ann Surg. 2004;240:205-213.

14. Riss S, Weiser FA, Schwameis K, et al. The prevalence of hemorrhoids in adults. Int J Colorectal Dis. 2012;27:215-220.

15. Lohsiriwat V. Approach to hemorrhoids. Curr Gastroenterol Rep. 2013;15:332-013-0332-6.

16. Longo A. Treatment of hemorrhoidal disease by reduction of mucosa and hemorrhoidal prolapse with a circular-suturing device: a new procedure. In: Proceedings of the 6th World Congress of Endoscopic. Rome, Italy: Surgery; 1998. 
17. Burch J, Epstein D, Sari AB, et al. Stapled haemorrhoidopexy for the treatment of haemorrhoids: a systematic review. Colorectal Dis. 2009;11:233-43; discussion 243.

18. Pavlidis T, Papaziogas B, Souparis A, Patsas A, Koutelidakis I, Papaziogas T. Modern stapled Longo procedure vs. conventional Milligan-Morgan hemorrhoidectomy: a randomized controlled trial. Int J Colorectal Dis. 2002;17:50-53.

19. Shalaby R, Desoky A. Randomized clinical trial of stapled versus Milligan-Morgan haemorrhoidectomy. Br J Surg. 2001;88:1049-1053.

20. Senagore AJ, Singer M, Abcarian H, et al. A prospective, randomized, controlled multicenter trial comparing stapled hemorrhoidopexy and Ferguson hemorrhoidectomy: perioperative and oneyear results. Dis Colon Rectum. 2004;47:1824-1836.

21. Gravie JF, Lehur PA, Huten N, et al. Stapled hemorrhoidopexy versus milligan-morgan hemorrhoidectomy: a prospective, randomized, multicenter trial with 2-year postoperative follow up. Ann Surg. 2005;242:29-35.

22. Hetzer FH, Demartines N, Handschin AE, Clavien PA. Stapled vs excision hemorrhoidectomy: longterm results of a prospective randomized trial. Arch Surg. 2002;137:337-340.

23. Festen S, Molthof H, van Geloven AA, Luchters S, Gerhards MF. Predictors of recurrence of prolapse after procedure for prolapse and haemorrhoids. Colorectal Dis. 2012;14:989-996.

24. Collinson R, Wijffels N, Cunningham C, Lindsey I. Laparoscopic ventral rectopexy for internal rectal prolapse: short-term functional results. Colorectal Dis. 2010;12:97-104.

25. Boons P, Collinson R, Cunningham C, Lindsey I. Laparoscopic ventral rectopexy for external rectal prolapse improves constipation and avoids de novo constipation. Colorectal Dis. 2010;12:526532.

26. Slawik S, Soulsby R, Carter H, Payne H, Dixon AR. Laparoscopic ventral rectopexy, posterior colporrhaphy and vaginal sacrocolpopexy for the treatment of recto-genital prolapse and mechanical outlet obstruction. Colorectal Dis. 2008;10:138-143.

27. Grigoropoulos P, Kalles V, Papapanagiotou I, et al. Early and late complications of stapled haemorrhoidopexy: a 6-year experience from a single surgical clinic. Tech Coloproctol. 2011;15 Suppl 1:S79-81.

28. Tjandra JJ, Chan MK. Systematic review on the procedure for prolapse and hemorrhoids (stapled hemorrhoidopexy). Dis Colon Rectum. 2007;50:878-892.

29. Oughriss M, Yver R, Faucheron JL. Complications of stapled hemorrhoidectomy: a French multicentric study. Gastroenterol Clin Biol. 2005;29:429-433.

30. Pescatori M, Gagliardi G. Postoperative complications after procedure for prolapsed hemorrhoids (PPH) and stapled transanal rectal resection (STARR) procedures. Tech Coloproctol. 2008;12:7-19.

31. Pigot F, Dao-Quang M, Castinel A, et al. Low hemorrhoidopexy staple line does not improve results and increases risk for incontinence. Tech Coloproctol. 2006;10:329-333. 
32. Correa-Rovelo JM, Tellez O, Obregon L, et al. Prospective study of factors affecting postoperative pain and symptom persistence after stapled rectal mucosectomy for hemorrhoids: a need for preservation of squamous epithelium. Dis Colon Rectum. 2003;46:955-962.

33. Ommer A, Hinrichs J, Mollenberg H, Marla B, Walz MK. Long-term results after stapled hemorrhoidopexy: a prospective study with a 6-year follow-up. Dis Colon Rectum. 2011;54:601608.

34. Finco C, Sarzo G, Savastano S, Degregori S, Merigliano S. Stapled haemorrhoidopexy in fourth degree haemorrhoidal prolapse: is it worthwhile? Colorectal Dis. 2006;8:130-134.

35. Arroyo A, Perez-Legaz J, Miranda E, et al. Long-term clinical results of double-pursestring stapled hemorrhoidopexy in a selected group of patients for the treatment of chronic hemorrhoids. Dis Colon Rectum. 2011;54:609-614.

36. Chen JS, You JF. Current status of surgical treatment for hemorrhoids--systematic review and metaanalysis. Chang Gung Med J. 2010;33:488-500.

37. Herold A, Kirsch JJ. Pain after stapled haemorrhoidectomy. Lancet. 2000;356:2187; author reply 2190 . 


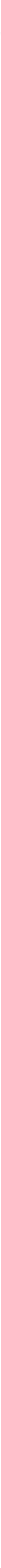




\section{SECTION 2}

Future perspectives in surgery for rectal prolapse 


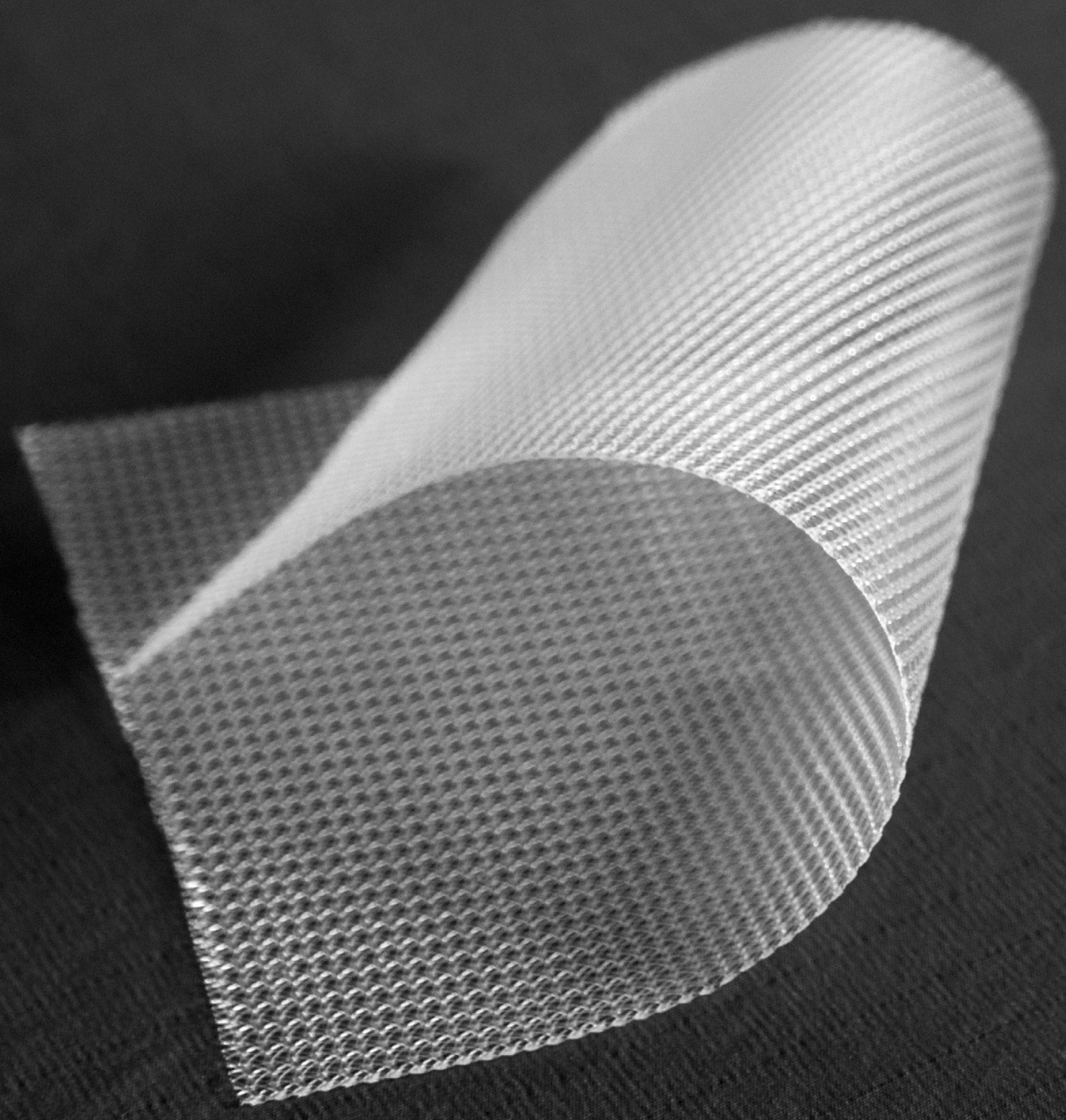




\section{Chapter 7}

\section{The optimal strategy for proximal mesh fixation during laparoscopic ventral rectopexy for rectal prolapse: an ex-vivo study}


Aim

Laparoscopic ventral rectopexy (LVR) is an established technique for the treatment of rectal prolapse. Several techniques and devices can be used for proximal mesh fixation on the sacral promontory during this procedure. The aim of this study was to compare the fixation strength of a recently introduced screw for mesh fixation on the promontory during LVR with two other frequently used techniques.

\section{Methods}

An ex vivo experimental model using a porcine spinal column was designed to measure the strength of proximal mesh fixation. In a laparoscopic box trainer, a polypropylene mesh was anchored on the spinal column using three different fixation methods, i.e., the Protack 5-mm tacker device, Ethibond Excel 2-0 stitches, and the Karl Storz screw. Subsequently, increasing traction was applied to the mesh. This traction was applied at a standardized angle as determined by measuring the mean angle between the site of distal mesh fixation on the rectum and a line straight through the sacral promontory on 12 random dynamic MR scans of the pelvic floor after the LVR procedure. The applied force was measured at the moment that the fixation broke, using a calibrated electronic Newton meter. All fixation methods were tested ten times.

\section{Results}

The mean angle, as measured on the MR scans, was $100^{\circ}$. The mean disruption force, which led to a break of the proximal mesh fixation, was $58 \mathrm{~N}$ for the three Protack tacks, $55 \mathrm{~N}$ for the two stitches, and $70 \mathrm{~N}$ for the new screw. The use of a screw therefore led to a significantly stronger fixation compared to the use of stitches ( $p \leq$ $0.05)$. No significant difference was determined between the tacks and the screw fixation and between the tacks and the stitches fixation.

\section{Conclusion}

The new screw for proximal mesh fixation during LVR procedures offers similar fixation strength when compared to tacks. The use of one screw for proximal mesh fixation is therefore a reasonable alternative to the use of several tacks or sutures. 


\section{INTRODUCTION}

Rectal prolapse (RP) is the full-thickness descent of the upper rectum [1]. This can be either internal (intussusception) or external, in which internal RP is thought to be the predecessor of an external prolapse [2]. RP may cause constipation, fecal incontinence, or the combination of these symptoms in the vast majority of patients, and, therefore, treatment is often necessary [3]. Laparoscopic ventral rectopexy (LVR) is an established surgical technique for the treatment of patients with an internal or external rectal prolapse. Several studies have been published demonstrating satisfactory subjective and functional outcomes after LVR [4-10]. This has led to implementation of LVR as the surgical strategy of choice by many pelvic floor surgeons, especially in Europe. During LVR, the distal rectum is mobilized ventrally by dissection of the rectovaginal planum, after which a non-resorbable mesh is fixed on the ventral side of the distal rectum. Following distal attachment, the mesh is attached proximally onto the sacral promontory [7].Several methods are used for the proximal attachment of the mesh on the sacral promontory. In the original articles describing the procedure, the use of either sutures or an endofascia stapler was described $[6,7]$. Nowadays, the most popular methods for this fixation are non-resorbable stitches or tacks made of titanium. Recently, a titanium screw (Karl Storz, Tuttlingen, Germany) has been developed for proximal fixation of the mesh during LVR. This 2-mm $\times 8-\mathrm{mm}$ screw is designed with the objective to provide a secure, reproducible, and relatively inexpensive strategy for mesh fixation. The aim of this study was to evaluate and compare the strength of mesh attachment provided by the new screw, titanium tacks, and non-resorbable stitches for proximal mesh fixation during LVR in an experimental study design.

\section{MATERIALS AND METHODS}

\section{Experimental set-up}

A prospective comparative experimental study was designed to measure the fixation strength of the three different methods of proximal mesh fixation. A spinal column of a 6-month-old, 90-kg porcine corpse was used for this study. This animal was kept under routine laboratory conditions at the Central Animal Laboratory of the University of Nijmegen, the Netherlands. The pig was killed using $10 \mathrm{ml}$ of sodium pentobarbital $(200 \mathrm{mg} / \mathrm{ml})$ on the day of the experiment, after appropriate medical ethics approval. During dissection of the corpse, an en bloc resection of the spinal column and surrounding ligaments was performed. Polypropylene meshes (Prolene, Ethicon Inc., Johnson \& Johnson, Somerville, NJ, USA) measuring $3 \times 15 \mathrm{~cm}$ were used in this study, as this is the standard mesh for rectopexy in many institutions. The proximal end of the 
mesh was attached to the spinal column using one of three investigated fixation techniques: (1) two non-resorbable stitches (Ethibond Excel 2-0, Ethicon Inc.), (2) three titanium helix-shaped tacks (Autosuture Protack $5 \mathrm{~mm}$, Covidien, Mansfield, MA, USA) or (3) one titanium, 2-mm $\times$ 8-mm screw (Karl Storz, Tuttlingen, Germany). The placement of the three attachment methods was equally divided over the length of the spinal column. The position of mesh fixation techniques is illustrated in Figure 1. All fixation techniques were performed in a laparoscopic trainer box by an experienced laparoscopic surgeon (IB).

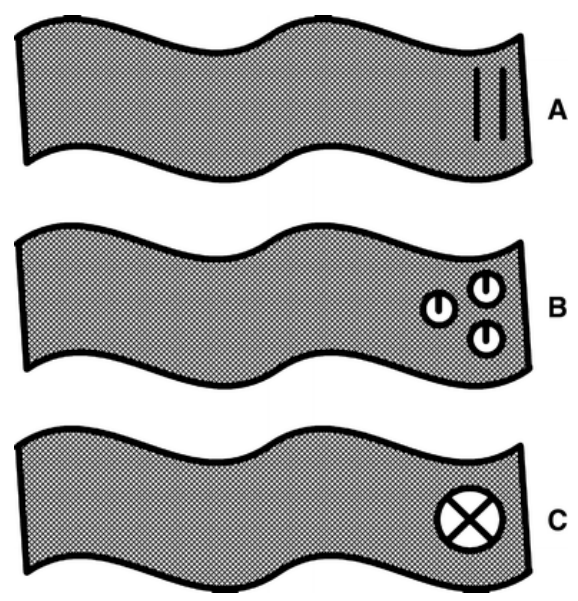

Figure 1 Configurations of (A) stitches, (B) tacks, and (C) the new screw on the mesh

\section{Determination of the in-vivo angle between sacral promontory and rectum}

To determine the in vivo angle between the sacral promontory and the point of distal mesh fixation on the rectum, all postoperative dynamic MRI (DMRI) studies of the pelvic floor after LVR were identified in the radiology department's Patient Archiving and Communication System (PACS). The angle between the site of mesh fixation on the sacral promontory and the distal attachment on the rectum was determined by measuring this angle between two lines. The first line was set straight on the site of fixation, through the sacral promontory. The second line was drawn between this site and the distal attachment of the mesh. The mean angle was determined and named A (Figure 2). All measurements were performed by an experienced radiologist $(\mathrm{BH})$. 

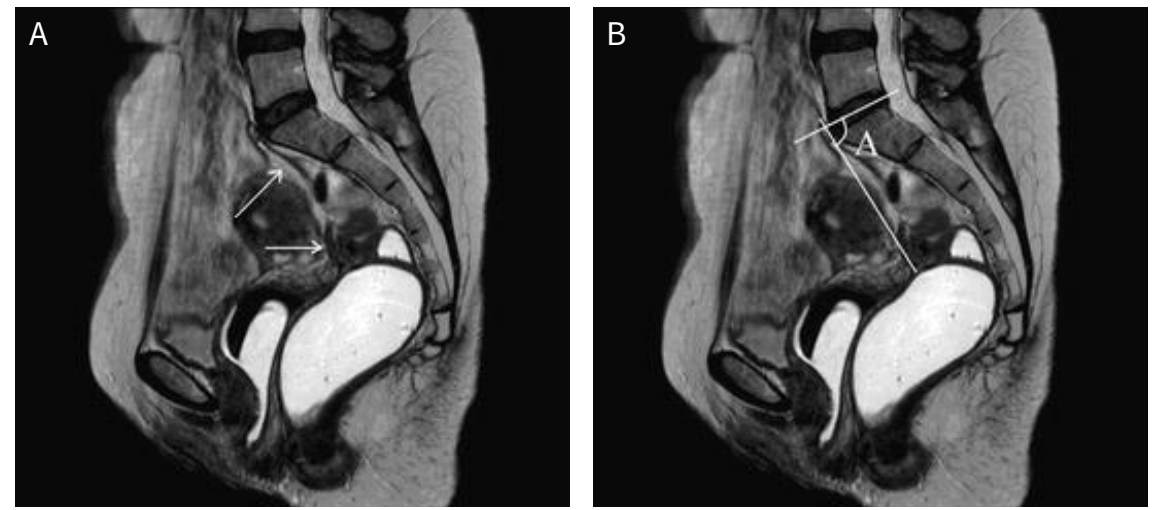

Figure $\mathbf{2 A}$ The postoperative MRI image of a 48-year-old female patient. This MRI was performed 8 weeks after laparoscopic ventral rectopexy, using tacks to fix the polypropylene mesh on the sacral promontory. Contrast gel has been inserted in the rectum and vagina. The polypropylene mesh can be followed from the sacral promontory to the first distal attachment point on the rectal wall, see white arrows. B By drawing a line over the spinal disc between L5 and S1 and a line from the mesh attachment point to the most proximal attachment point on the rectal wall, the angle of force (A) can be measured. In this case, it was $95^{\circ}$

\section{Measurement of outcome parameters}

A three-strand twisted natural-fiber rope (approximately $5 \mathrm{~mm}$ in diameter) was attached to the distal $4 \mathrm{~cm}$ of the mesh using multiple standard-issue staples. This was done using the full distance across the distal mesh to guarantee equal traction on both sides. A $10-\mathrm{cm}$, friction-free, industrial pulley was used to lead this cord in a vertical direction. A standard bucket (maximum content of $10 \mathrm{~L}$ ) was attached to the rope. Next, the bucket was filled with water at a constant rate of $20 \mathrm{~L} / \mathrm{min}$. Traction was performed in the mean angle "A" as determined on postoperative DMRI scans of patients who underwent LVR. The experimental setup is depicted in Figure $\mathbf{3}$.

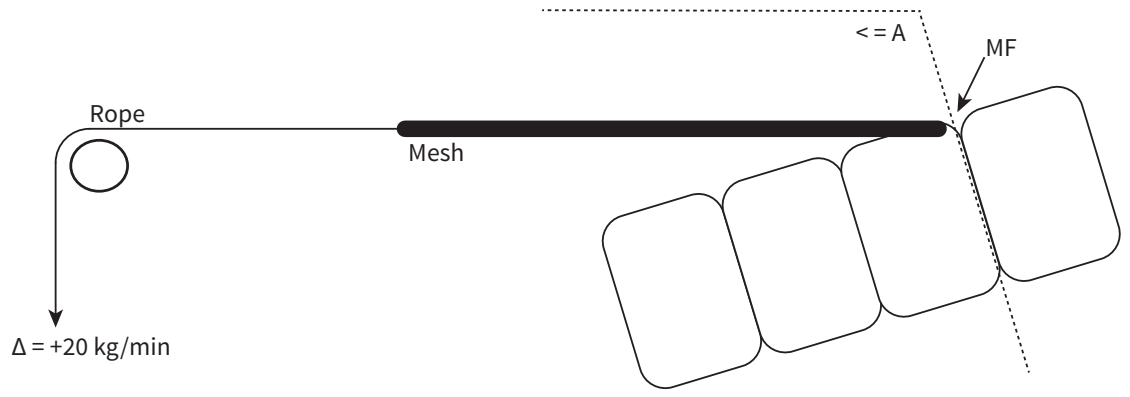

Figure 3 Schematic lateral illustration of experimental setup. The point of proximal mesh fixation (MF) is indicated by the arrow 
In case of disruption of the fixation, the water flow was stopped immediately. An electronic, calibrated, scientific spring suspension (Kern GmbH $100 \mathrm{~K} 200$ hanging scale, Kern \& Sohn, Balingen, Germany) was used to measure the total weight of the bucket, including its contents. The weight (in newtons [N]) was deemed the disruption force (DF). Furthermore, the exact mechanism of failure was noted. All fixation methods were tested ten times.

\section{Statistical analysis}

Statistical analysis SPSS version 17.0 (SPSS, Chicago, IL, USA) was used for statistical analysis. The data set was searched for outliers using a scatterplot. Outliers were excluded from statistical analysis using a scatterplot. A KolmogorovSmirnov test was performed to test normal distribution of the data. If normal distribution was confirmed, means per fixation method were determined. Student t-tests were used to determine significant differences between the various fixation methods. A p value of $<0.05$ was considered significant.

\section{RESULTS}

Eighteen patients who underwent a DMRI scan of the pelvic floor after LVR were found in our database. No rectal prolapse or intussusception recurrences and no anatomical variations or disorders were found. In six cases, the mesh could not be visualized adequately. In the 12 remaining DMRIs, the angle between the site of mesh fixation on the sacral promontory and the distal attachment on the rectum was determined (angle $A)$. The mean angle was $100.4^{\circ}\left(\right.$ range $\left.=93^{\circ}-112^{\circ}\right)$.

\section{Outcome parameters}

The results of the test with the sutures, tacks, and screw are given in table 1. The Kolmogorov-Smirnov test proved that the results were normally distributed for all three fixation methods

It was observed that during the ten tests with nonresorbable sutures, the ligamentum longitudinale of the spinal column ruptured in seven cases causing failure of the fixation of the mesh, but the sutures remained intact. In the other three cases, the sutures ruptured once; in one case the mesh tore around the sutures, and in the last case the placed knots in the sutures failed. The mean disruption force of the fixation with nonresorbable sutures was $55.2 \mathrm{~N}$ (range $=32-80, \mathrm{SD}=4.9$ ). In nine of the ten experiments with tacks, the mesh ruptured around the tacks, while the tacks stayed firmly anchored into the porcine spine. In the other case, both the tacks and the mesh broke out of the ligament. One outlier was determined in this data set and was excluded from analysis. The mean disruption force of tack fixation was $58.0 \mathrm{~N}$ (range $=38-76, \mathrm{SD}=14.0$ ). During all ten tests with one screw anchored to the spine, the screw remained lodged in the spinal disc and the mesh tore off around it. The mean disruption force was $70.0 \mathrm{~N}$ (range $=42-90, \mathrm{SD}=14.0$ ). 
Table 1. All results per fixation method

\begin{tabular}{lccc}
\hline Measurement no. & $\begin{array}{c}\text { 2 stitches } \\
\text { (Ethibond Excel 2-0) }\end{array}$ & $\begin{array}{c}\text { 3 tacks } \\
\text { (Protack) }\end{array}$ & $\begin{array}{c}\text { 1 screw } \\
\text { (Storz, 2 } \mathbf{~ m m ~} \times \mathbf{8 ~ m m} \text { ) }\end{array}$ \\
\hline 1 & 56 & 66 & 82 \\
2 & 60 & 74 & 80 \\
3 & 32 & 38 & 82 \\
4 & 56 & 74 & 42 \\
5 & 50 & 56 & 64 \\
6 & 70 & 44 & 62 \\
7 & 80 & 44 & 90 \\
8 & 46 & $116^{a}$ & 66 \\
9 & 66 & 50 & 70 \\
10 & 36 & 76 & 62 \\
Mean value & 55.2 & 58.0 & 70.0 \\
\hline
\end{tabular}

All results are given in newtons ( $N$ ), ${ }^{a}$ Outlying data, excluded from analysis

\section{Comparison of fixation methods}

The use of a screw for proximal mesh fixation proved to offer a significantly higher fixation strength compared to the use of stitches $(p=0.03)$. No significant differences were found between screw and tack fixation $(p=0.09)$ and between the use of tacks and stitches $(p=0.69)$.

\section{Discussion}

Laparoscopic ventral rectopexy is a widely used and accepted technique for the treatment of both internal and external rectal prolapse. This study compares the currently available techniques for mesh fixation in an ex vivo model and has demonstrated that both titanium tacks and a screw lead to favorable results when compared to nonresorbable stitches in terms of fixation strength.

The available literature on mesh fixation techniques during LVR for rectal prolapse surgery is scarce. In the original articles by D'Hoore et al. [6,7], the use of a polypropylene mesh is described, with distal fixation by nonresorbable sutures and proximal fixation by either nonresorbable sutures or an endofascia stapler. Since then, several articles [4, 5, 8-10] have reported on the results of LVR for both internal and external rectal prolapse. A polypropylene mesh is used in all these studies, and the majority of the studies mention the use of tacks as the proximal mesh fixation technique of choice. However, comparative studies on the optimal surgical strategy for mesh fixation at the site of the sacral promontory have never been performed until now. 
In the current study, an ex vivo experimental setup was chosen because in vivo studies on fixation strength techniques are hard to execute. It can therefore be argued whether the disruption forces measured in this experiment are relevant to in vivo use of these devices. Although it is possible to calculate the intra-abdominal pressure in humans, it is impracticable to determine the exact forces applied to the mesh after LVR [11]. In particular, the force on the mesh might be significant during straining and defecation, but these forces will remain unknown. Therefore, the current study is indicative of the optimal proximal mesh fixation although the forces of breakdown of the mesh may be unrealistic. It has been shown that all three fixation techniques offer adequate fixation of the mesh onto the sacral promontory. The disruption force using the screw, however, offers a significantly stronger fixation compared to the two stitches, and a trend to significance when compared to tacks. In the majority of tests, the mesh seemed to be the weakest point during tack and screw fixation. In almost all tests with the screw and the tacks, the mesh tore while the tacks and screw stayed firmly lodged in the promontory. Although the mesh proved to be the weakest link in both fixation methods, the mean results after screw fixation were higher compared to the tacks and sutures. This is probably caused by the distribution of forces around the site of fixation and is therefore a direct result of the fixation device that is used. During fixation with stitches, the ligamentum at the ventral side of the spinal column ruptured first. The stitches probably caused damage to the ligament thereby creating a relatively weak proximal fixation of the mesh.

When determining the optimal surgical strategy to use in LVR, several factors other than fixation strength will be important in the application of these techniques. Not only will the price affect the choice of fixation, but the ease of use and reproducibility also will be important factors. During the current study it was seen that minimal training was needed to adequately place the screw into the sacral promontory in a limited time frame when compared to the use of sutures. Furthermore, sutures have the disadvantage of user-dependent results because laparoscopic suturing demands extensive training. The tacks seemed to be the fastest and most easy-to-use method for mesh fixation; however, cost per operation of this disposable device are higher compared to that of the screw and much higher compared to stitching the mesh onto the sacral promontory. In summary, this study has demonstrated that the new screw for proximal mesh fixation during LVR offers strong fixation. The mesh fixation with the screw is significantly stronger compared to that of two stitches. Screw fixation is a cost-effective and reproducible method and this ex vivo study supports clinical implementation. 


\section{REFERENCES}

1. Wu JS (2009) Rectal prolapse: a historical perspective. Curr Probl Surg 46(8):602-716

2. Wijffels NA, Collinson R, Cunningham C, Lindsey I (2010) What is the natural history of internal rectal prolapse? Colorectal Dis 12(8):822-830

3. Madiba TE, Baig MK, Wexner SD (2005) Surgical management of rectal prolapse. Arch Surg 140(1):63-73

4. Boons P, Collinson R, Cunningham C, Lindsey I (2010) Laparoscopic ventral rectopexy for external rectal prolapse improves constipation and avoids de novo constipation. Colorectal Dis

5. Collinson R, Wijffels N, Cunningham C, Lindsey I (2010) Laparoscopic ventral rectopexy for internal rectal prolapse: short-term functional results. Colorectal Dis 12(2):97-104

6. D'Hoore A, Cadoni R, Penninckx F (2004) Long-term outcome of laparoscopic ventral rectopexy for total rectal prolapse. Br J Surg 91(11):1500-1505

7. D'Hoore A, Penninckx F (2006) Laparoscopic ventral recto(colpo)pexy for rectal prolapse: surgical technique and outcome for 109 patients. Surg Endosc 20(12):1919-1923

8. Slawik S, Soulsby R, Carter H, Payne H, Dixon AR (2008) Laparoscopic ventral rectopexy, posterior colporrhaphy and vaginal sacrocolpopexy for the treatment of recto-genital prolapse and mechanical outlet obstruction. Colorectal Dis 10(2):138-143

9. van den Esschert JW, van Geloven AA, Vermulst N, Groenedijk AG, de Wit LT, Gerhards MF (2008) Laparoscopic ventral rectopexy for obstructed defecation syndrome. Surg Endosc 22(12):27282732

10. Wijffels N, Cunningham C, Dixon A, Greenslade G, Lindsey I (2011) Laparoscopic ventral rectopexy for external rectal prolapse is safe and effective in the elderly. Does this make perineal procedures obsolete? Colorectal Dis 13(5):561-566

11. Cobb WS, Burns JM, Kercher KW, Matthews BD, James NH, Todd HB (2005) Normal intraabdominal pressure in healthy adults. J Surg Res 129(2):231-235 


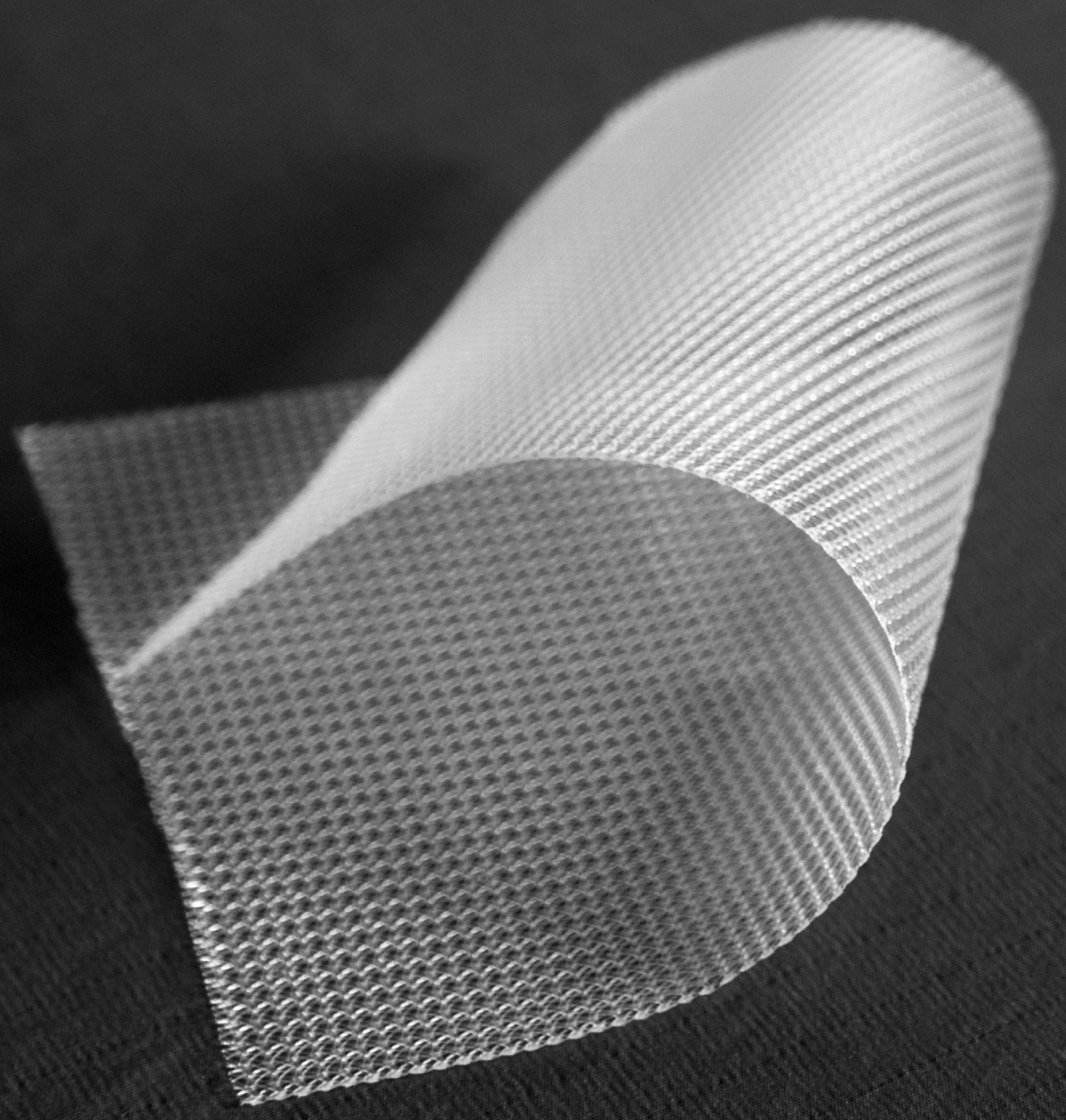




\section{Chapter 8}

\section{Robot Assisted Rectal Prolapse Repair}




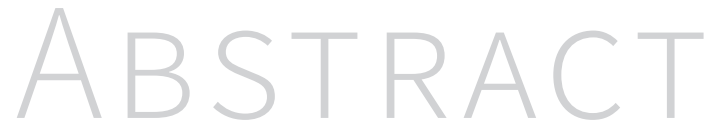

Rectal prolapse is the descent of the upper rectum and predominantly occurs in women older then the age of 50 . This disabling condition causes constipation and / or fecal incontinence in the majority of patients. As rectal prolapse is often part of a multi-organ pelvic floor problem it is essential to evaluate and treat all patients with a multidisciplinary team.

The only definite treatment for descent of the upper rectum is surgery. Numerous surgical techniques have been described in the past involving both perineal and abdominal approaches. In recent years, laparoscop- ic rectopexy procedures have gained popularity due to good functional results, low morbidity and a low recurrence rate. Today, laparoscopic ventral rectopexy is the treatment of choice for many surgeons.

Ventral rectopexy is increasingly performed with robotic-assistance. The use of the robot during this procedure is attractive to surgeons as it requires complex maneuvers deep in the narrow pelvis. Robotic-assisted ventral rectopexy is described in detail in this chapter and tips and pitfalls for this procedure are provided. 


\section{INTRODUCTION}

In rectal prolapse the upper part of the rectum descends. As long as the rectum stays within the anal ring the condition is called intussusception. In external prolapse the rectum protrudes through the anus. An internal prolapse is thought to be a precursor of external rectal prolapse. Both conditions may lead to obstructed defecation, fecal incontinence and mucosal ulceration. Although observed in both sexes and in all ages including children, the highest incidence of rectal prolapse occurs in women older than the age of 50. Rectal prolapse is usually part of a multi-organ pelvic floor disorder with concurrent prolapses of the bladder, uterus and vagina. [1-3]

A number of risk factors for rectal prolapse have been identified, such as a deep pouch of Douglas, lax lateral rectal ligaments, weakness of the internal and external anal sphincters and obstetric trauma like episiotomy. Furthermore, multiparous women seem more prone to develop a rectal prolapse. A long history of excessive straining, neurologic disorders and constipation may also contribute to the development of a rectal prolapse. $[1,4]$

There is a role for non-surgical treatment of internal prolapse. Medication may consist of bulking agents, stool softeners and suppositories or enemas. Biofeedback training of the pelvic floor might be beneficial for some patients in order to improve control over the pelvic floor muscles.

However, the only definite treatment for rectal prolapse is surgery. Numerous procedures have been proposed including perineal access techniques and techniques with an abdominal approach by laparotomy or laparoscopy. $[1,5,6]$ Robotic surgery may further refine the technique of endoscopic rectal prolapse repair because this procedure requires complex visualization of a narrow and distant operating field, delicate dissection and extensive suturing. This chapter provides general information on rectal prolapse and detailed explanation on the technique of robot assisted ventral rectal prolapse repair.

\section{Surgical Repair Of Rectal Prolapse}

More than 400 surgical techniques for rectal prolapse surgery have been described in the literature. In general these techniques are used to either obliterate the pouch of Douglas, to narrow the anal canal, restore the pelvic floor, resect the redundant bowel, or to suspend or fixate the rectum to the sacrum or other structures.[6]

Less recurrence is observed with the abdominal approach compared to peranal or perineal procedures. Abdominal techniques should therefore be offered to all patients except those who are not fit for general anesthesia. These can be operated on with perineal techniques under spinal anesthesia. [1,5] With 
modern anesthesiology and endoscopic surgical techniques, patients unsuitable for an abdominal approach are rarely seen. Sometimes limited additional treatment of mucosal prolapse is required after abdominal repair of full thickness prolapse.

Abdominal procedures were initially performed via laparotomy. Both the Frykman-Goldberg resection rectopexy and the ventral rectopexy as decribed by D'Hoore have been modified for a laparoscopic approach. [5, 7-9, 13] The use of robotic assistance has been described in several case series. [10-12] The ventral rectopexy appears to be the procedure of preference in Europe, while resection rectopexy is generally applied in the USA. Theoretically, resection rectopexy may be favorable in treating constipation, with a relatively small risk of anastomotic leakage.

\section{Signs ANd COMplaints Of Rectal Prolapse}

Clinical assessment of patients suspected of rectal prolapse should include a comprehensive medical history and a thorough physical examination and rectal inspection. In female patients, a gynecologist and urologist should be consulted to evaluate the middle and anterior compartment of the pelvic floor for concurrent prolapses. In case of suspicion of prolapse radiological examination like evacuation proctography or dynamic magnetic resonance imaging (D-MRI) of the pelvic floor should be obtained. With these investigations rectal function and prolapse are analyzed during straining and defecation, as these maneuvers are likely to enlarge prolapse. Anal endosonography and anal manometry are not part of standard pre-operative evaluation. These are used to determine possible sphincter defects and neurologic anorectal function and should be used in case of suspicion of disorders other than prolapse.

It is advisable to present patients with pelvic floor disorders in a multidisciplinary setting in order to provide a tailored surgical approach. This multidisciplinary team should consist of (colorectal) surgeons, gynecologists and urologists, radiologists and pelvic floor physical therapists.

\section{Medical History and Physical Examination}

Important aspects of a patient's medical history are the number of deliveries and possible complications like sphincter ruptures, episiotomy and excessive straining. It is also essential to determine the number of years of constipation and its severity, as well as the presence or absence of fecal incontinence. Actual fecal incontinence has to be distinguished from soiling during a prolapsed state. Furthermore, the frequency and severity of incontinence and the consistency of stool during incontinence has to be questioned. The use of standardized questionnaires (e.g. Cleveland Clinic Incontinence Score and Cleveland 
Clinic Constipation Score) are recommended to assess symptoms and to evaluate the effect of treatment.

When examining the perineal area, inspection should include presence of scars, fistulas and hemorrhoids. A gaping anus may indicate recurrent rectal prolapse. Straining during examination often increases mucosal or rectal prolapse. Uterine and bladder prolapse should be documented. Digital rectal examination is an essential part of physical examination and is performed to detect sphincter defects, scars, and to determine the consistency of feces in the rectal ampulla.

\section{Proctoscopy}

Proctoscopy is used to assess the rectum for additional lesions like solitary rectal ulcers. These can be found in up to $25 \%$ of patients with internal or external rectal prolapse and appear as ulceration on the anterior rectal wall. Biopsies should be taken to exclude other pathology. A complete colonoscopy should be considered in case of blood loss or a recent change in the defecation pattern.

\section{Anorectal Endosonography}

Anorectal endosonography provides anatomical images of the anal sphincters and pelvic floor. Defects of both the internal and external sphincters can be demonstrated using this technique. Scars and atrophy can also be visualized. It is advised in cases where incontinence is suspected to be caused by sphincter defects.

\section{Anal Manometry}

Anal manometry evaluates the function of the anal sphincter muscles during rest and straining by measuring the pressure in the anal canal. Physiological basal anal sphincter pressure is mainly generated by the internal anal sphincter and in lesser degree by the external anal sphincters and hemorrhoidal plexus. The range of normal values is wide and mostly influenced by age and gender. In the vast majority of patients with a rectal prolapse a decreased sphincter pressure is found. The clinical significance is unclear and anal manometry rarely influences the indication for surgery or type of surgical technique.

\section{Radiologic Investigation}

Evacuation defecography used to be the investigation of choice in the radiologic assessment of patients with symptoms of rectal prolapse. The patient is asked to defecate on a special radiolucent toilet after rectal and vaginal instillation of a radiopaque substance. A series of images results in display of defecation over a short period of time. Furthermore it is possible to determine the anorectal angle, the puborectalis muscle relaxation and vaginal vault prolapse. Because evacuation defecography exposes the patient to a considerable amount of radiation, dynamic magnetic resonance imaging (D-MRI) has become an alter- 
native technique for evaluation of the pelvic floor. D-MRI provides comprehensive information about all compartments of the pelvic floor, in the mid-sagital plane. A drawback of dynamic MRI is the supine position during investigation in most MRI scanners. The choice for one of these two imaging techniques depends on local availability and costs.

\section{INDICATIONS FOR SURGERY}

The Oxford rectal prolapse classification is widely used for assessment and evaluation of rectal prolapse.[14] The presence of full thickness external rectal prolapse (Oxford grade V) during physical examination or on imaging studies (evacuation defecopgraphy or dynamic MRI) is a clear indication for surgery. Internal rectal prolapse (Oxford grade III-IV) can be an indication for surgical intervention as well after failure of conservative treatment. Prior to surgery, rectal intussusception has to be observed during imaging studies and there has to be a correlation between symptoms of incontinence and constipation and observed prolapse.

\section{Surgical Technique of Robotic-Assisted Ventral Rectopexy (RVR) PREPARATION}

The patient receives an enema in the hours preceding surgery. The procedure is performed under general anesthesia because of the pneumoperitoneum and the sometimes steep Trendelenburg position during surgery. There is no need for epidural anesthesia for per- and postoperative pain relief because postoperative pain is limited.

The legs are positioned in leg rest to allow rectal investigation during surgery. The upper legs should be positioned as low as possible, but compression of ischiadic- and peroneal nerves or stretching of femoral nerves should be avoided at all times. This should be tested before draping, in neutral- and Trendelenburg position because the latter may result in a shift of the patient body in the direction of the head of the table. This shift should be limited by shoulder support. We use a head rest for this purpose (Figure 1). This headrest allows a natural position of the neck, and a soft support of the shoulders that optimally divides compression to avoid neurologic- or soft tissue damage. The body can be immobilized further by using straps or a half bean bag mattress (Figure 2). A urinary catheter is placed.

The patients head should be protected against contact with the camera head. This can be done by a standard metal bar in a low position. We use two standard bars at different height, and developed a plexiglas frame that rests on these bars. This frame serves two purposes: it allows good access to the well 


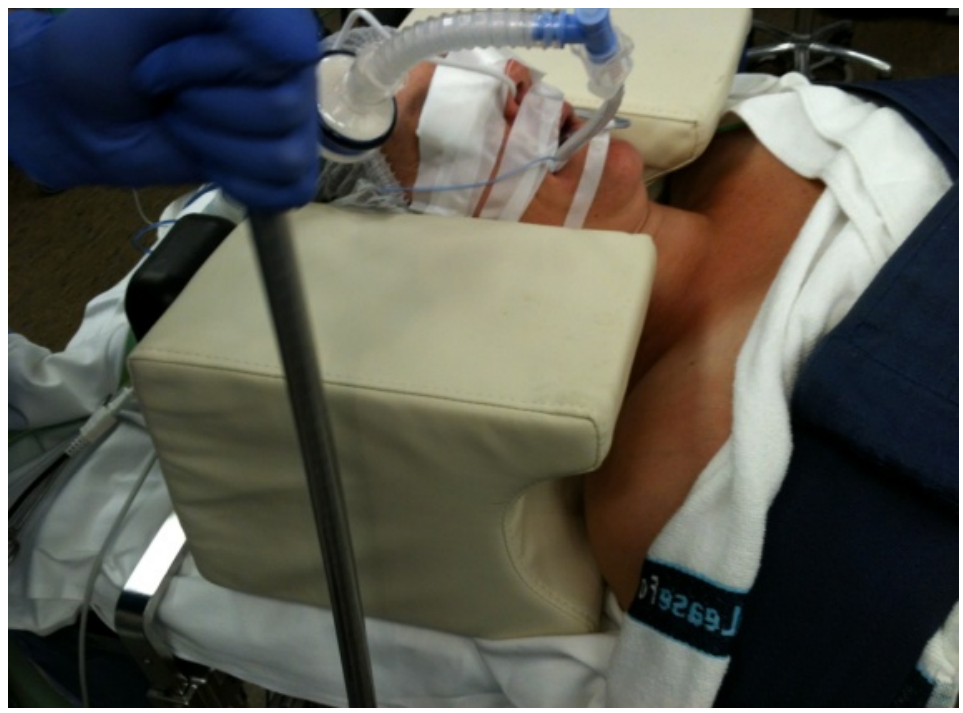

Figure 1. Head rest for steep Trendelenburg.

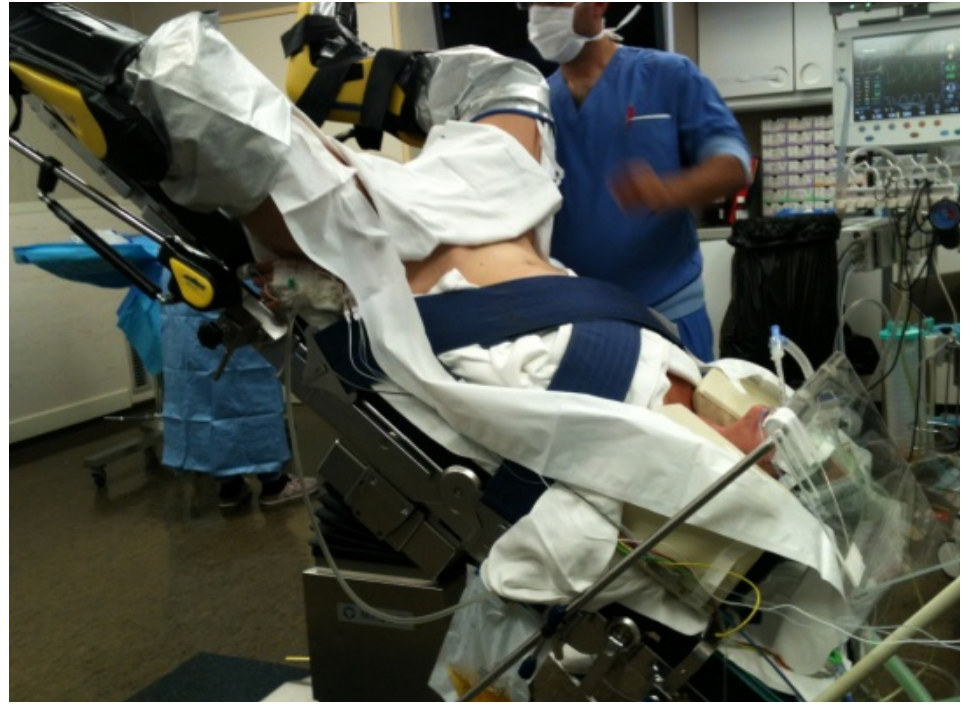

Figure 2. Straps to prevent body shift.

protected patients head by the anesthesiologist, and created a rest for the camera and optic in the sterile field after draping (Figure 3 ). The patient's right arm rests on an arm support to allow adequate intravenous access. The left arm is positioned alongside the body. Draping should allow rectal examination during surgery. 

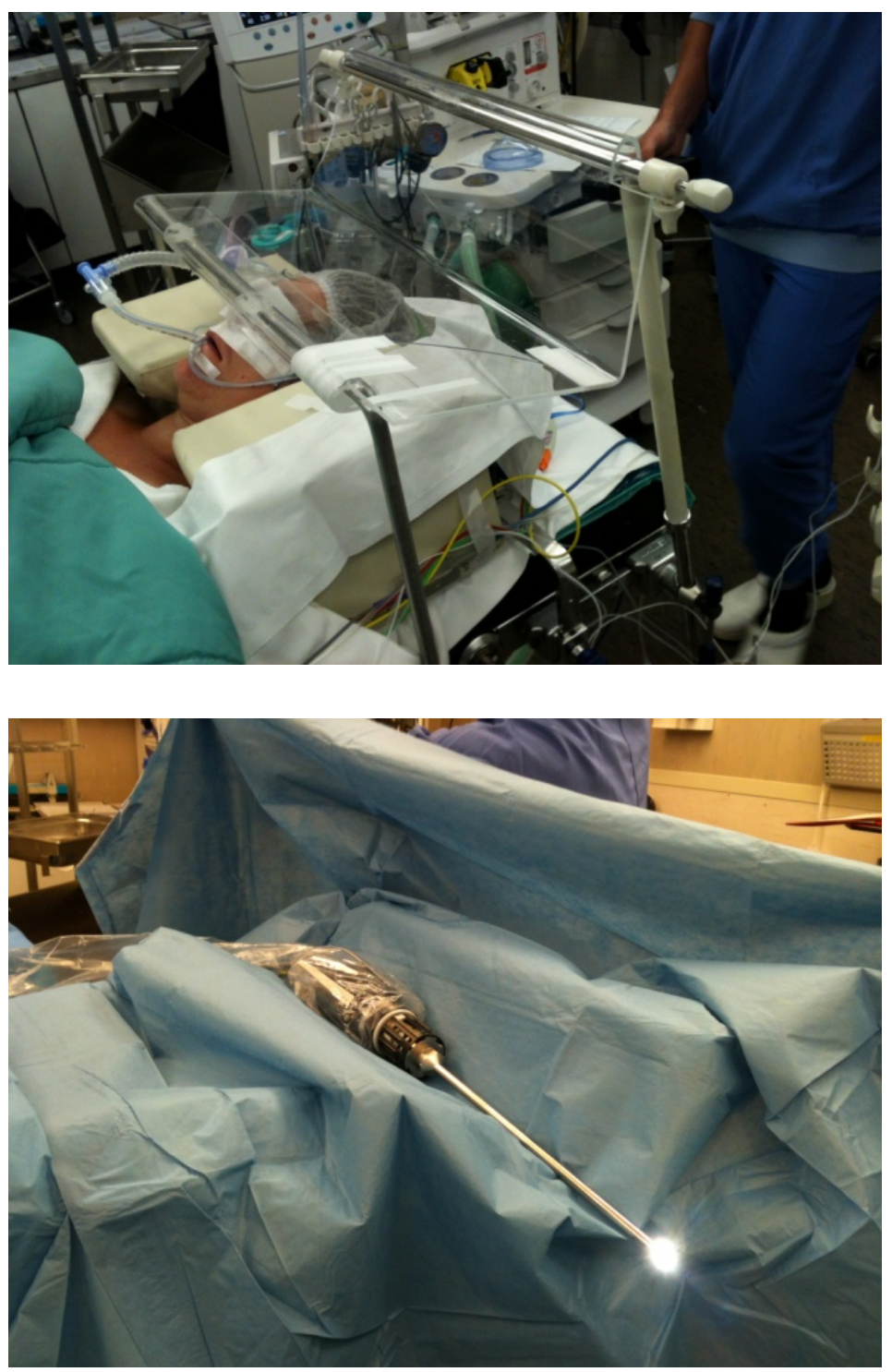

Figure 3. Plexiglas table for patient protection and camera rest. 

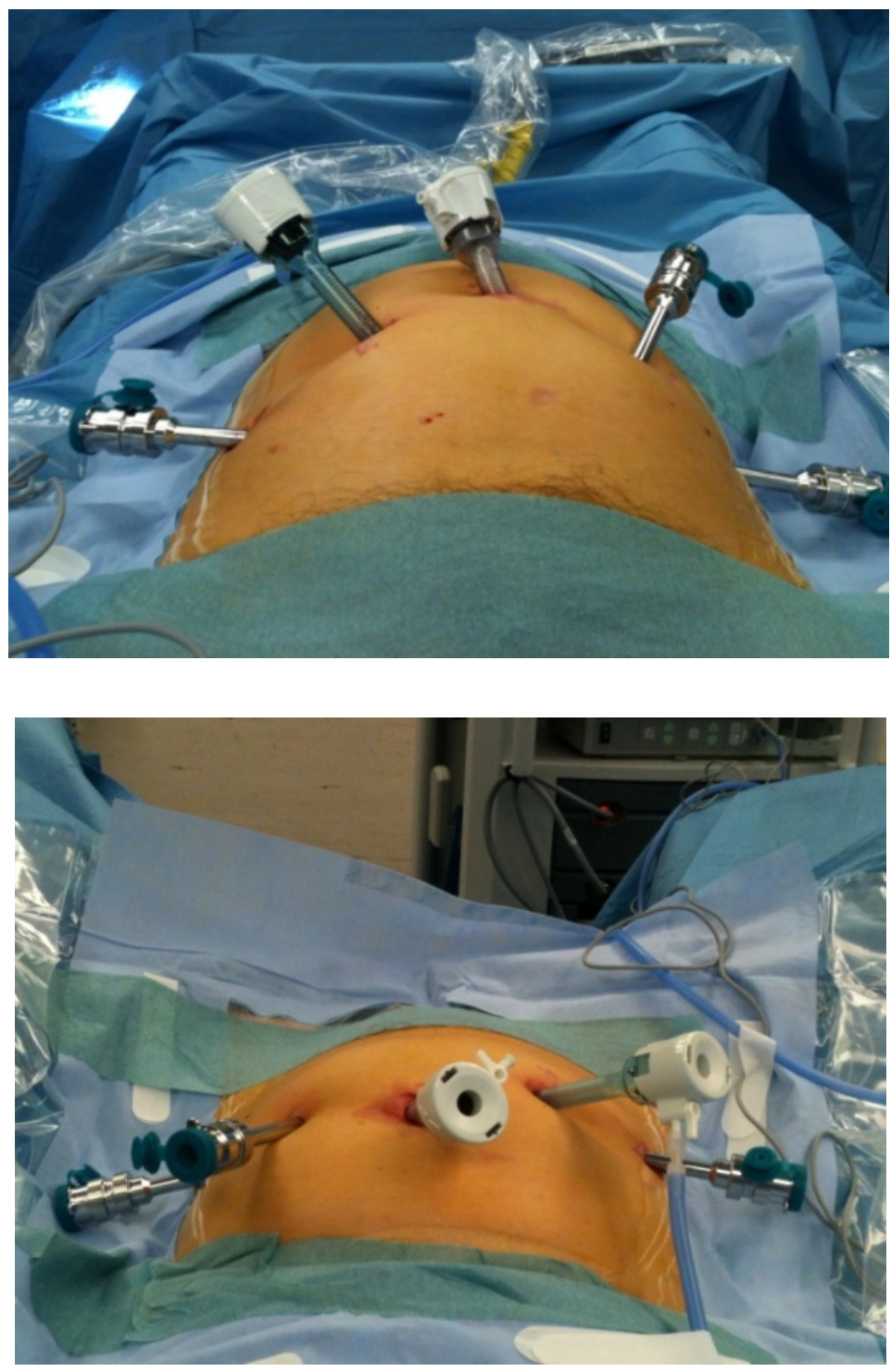

Figure 4. Trocars inserted. 


\section{Positioning of the Trocars}

Correct positioning of the trocars is of great importance to avoid contact between the robotic arms and to allow free access of the instruments in the pelvic area. The incision for the camera trocar is placed in the upper rim of the umbilicus. This provides enough distance and results in an invisible scar. The robotic arm trocars are placed along a fluent "negative smile" line. Trocars for arms number one and three are placed as laterally as possible, close to the iliac spine. The trocar for arm number two is placed on the left side centrally on the line between trocars for arm number three and the camera arm. The assistant port is placed centrally on the right side between the camera arm trocar and the trocar for arm number one. The assistant port may be placed caudally to the aforementioned line to allow a more perpendicular approach to the sacral promotorium during stapling (Figure 4). Avoid more caudal placement of the robotic arm trocars because this will result in excessive movements of the robotic arm outside the body. Be sure not to position arm in trocar two in front of arm trocar three because this will result in arm collision. Trocars are placed with the optic in a 30 degrees upward position. After trocar placement the optic is turned to 30 degrees downward and the table is positioned in steep Trendelenburg.

Any adhesions of the sigmoid colon or small intestine that hinder access to the pelvic cavity are cut with standard laparoscopic scissors. When the pelvic cavity and sacral promotory area are freed from small intestine and the loop of the sigmoid colon, the table can be repositioned to a less steep Trendelenburg position that still allows the view of the operative field as mentioned.

\section{Coupling of the Robot}

Ideally, the robot is positioned between the patients legs but this severely hinders rectal examination. The robotic cart is therefore positioned at the lower left side of the OR table. The angle between the axis of the table and the axis of the cart should be limited, usually between 20 and 30 degrees. The patient's legs should be spread as little as possible to accommodate this position. While approaching, the surgeon takes and frees the camera arm and pulls this arm forward. The cart is approached until the arm marker on the camera arm enters the blue zone. Arm number one is pulled over the right leg and is positioned with the joint in a relatively horizontal position facing to the right (Figure 5). Arm two is coupled in a relative vertical position and arm three is coupled in a nearly horizontal position with the joint facing to the left. This position of the arms allows optimal freedom and avoids collision. Be careful not to compress the left upper leg with arm number three. 

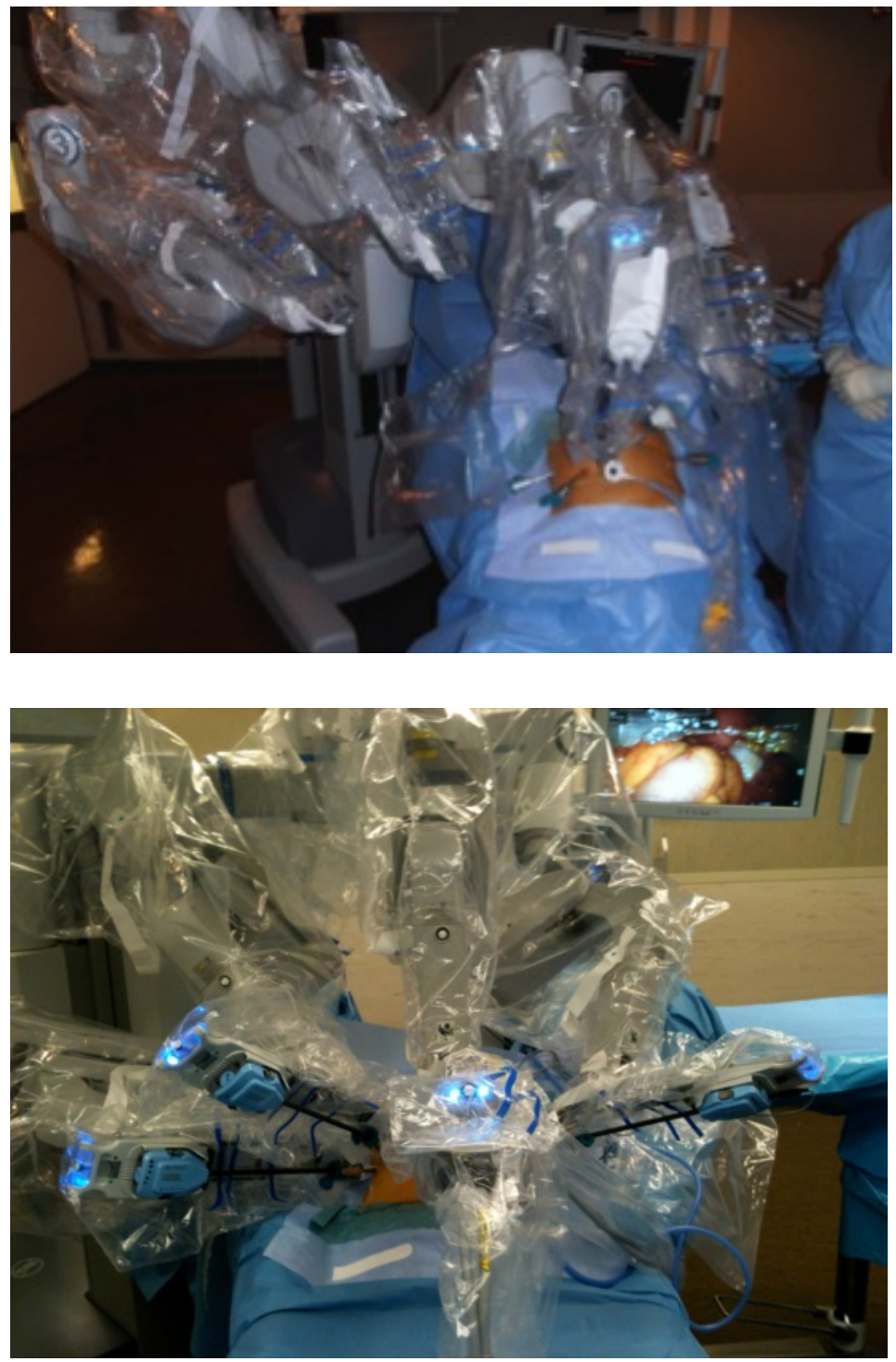

Figure 5. Robot approach and coupling. 


\section{Robot-Assisted Ventral Rectopexy}

If a uterus is present, this organ should be fixed to the upper abdominal wall. A grasping forceps is introduced at arm three, and a suture cut needle drive at arm one. A long straight needle with a thick monofilament wire is introduced by the assistant just above the pubic bone.The needle perforates the uterus at the top in an upward direction and the needle is brought outward close to the introduction spot. This needle is grabbed by the assistant and the two instruments are used to lift the uterus to the upper abdominal wall. The assistant ties the suture ends over a rolled gauze to avoid scarring of the underlying skin.

A second grasping forceps or a Cadiere forceps is introduced at arm 3. This forceps can be used to pull the rectum in the cephaled direction, or to lift the vagina. These tasks are divided cooperativley with the assistant who uses the assistant port for introducing a grasper or suction device. A cautery hook, or, if preferred, a coagulating scissors is introduced at arm 1 . A J-shaped incision is made at the deepest level of the pelvic cavity. Be sure to retract the rectum to allow optimal visualization of the Douglas pouch. The longer leg may be cut now up to the level of the promotorium but one can also decide to focus on rectal dissection first, and free the promotory later. The long leg of the incision should be positioned alongside the border of the perirectal fat. More lateral dissection should be avoided to stay away from the ureters. The underlying tissue beneath the peritoneal incision is divided in a merely blunt fashion to create space for the mesh. More extensive peritoneal dissection is advised in the horizontal part of the incision. Peritoneal closure taking big bites of peritoneal after mesh placement will result in a less deep pelvic cavity.

Freeing the sacral promotory is done with great care and small steps to avoid damage to the hypogastric plexus. This plexus can usually be discriminated well. Gas dissection is helpful at this point to split the fibrous tissue layers that cover the nerves and the promotory. The sacral artery is found and spared. When dissecting too proximal one will encounter the left iliac vein.

In case of patients with a high body mass index, it may be difficult to estimate the position of the promotory because palpation with the instruments does not result in haptic feedback. One can ask the assistant to palpate the position of the promotory. In case of doubt one should open the hole plane as in rectal resection, and follow this route to visualize the promotory.

Dissection of the recto-vaginal plane can be performed very well with the cautery hook, using the angle of the hook for blunt dissection, and the tip for cauterization of small vessels and fibrous stains. Others may prefer a monopolaror bipolar scissors. The third arm should be used to lift the vagina. The assistant port is used to pull the rectum in cephalic direction. Great care should be taken to dissect in a bloodless manner. Infiltration of the fatty tissue by blood makes it hard to discriminate the longitudinal fibers of the ventral rectal wall. 
When opening the recto-vaginal plane dissection is performed at the level on the ventral rectal wall to avoid vessels at the dorsal vaginal wall. Good retraction of the vagina facilitates blunt dissection in this plane. Dissection lateral to the muscular tube should not be performed beyond the upper level of the rectum to avoid nerval damage. The rectovaginal plane is dissected to the level of the pelvic floor. This requires repetitive repositioning of the third arm in order to grab the dorsal vaginal wall at a deeper position. This dissection can usually be performed with the scope in a 30 degrees downward position, but in about one in three cases it is necessary to change to 30 degrees upward to visualize the pelvic floor area. If the dissection of the recto-vaginal plane becomes difficult and the pelvic floor muscles are not clearly visualized, one can position a grasper at the deepest point of dissection, leave the console and perform rectal examination. The tip of the instrument should be felt no further than $2-4 \mathrm{~cm}$ measured from the outer anal aspect.

When dissection is completed the mesh can be introduced. Various types of meshes have been proposed, but a non absorbable mesh is preferable for a durable result. We use a straightforward polypropylene 10 by $15 \mathrm{~cm}$ mesh and cut a 3 by $15 \mathrm{~cm}$ strip. Mesh fixation is started at the deepest point, taking the pelvic floor at the upper border of the rectum. At least six non-absorbable sutures are used to fix the mesh to the anterior rectal wall, both in the midline and along the sides of the anterior aspect of the preperitoneal rectal wall. One should try to avoid full thickness sutures because of bacterial contamination. The high magnification 3-D view is very helpful in discriminating thickness of the bites. Usually 7-10 knots are tied with braid sutures. A $15 \mathrm{~cm}$ wire and a suture-cut needle driver allow the placement of three knots without changing instruments, wires and needles. One can use two needle drivers, but often rectal traction is applied to place stitches in the deeper areas and this requires grasping forceps or Cadiere forceps as the primary assisting instrument.

The mesh is subsequently fixed to the sacral promotory. Robotic arm three is used to pull the rectal mesentery to the left to free the area of the promotory. The mesh is positioned with a grasper in arm one and the hypogastric plexus is secured laterally with a grasper in arm two. The mesh can be fixed using non-absorbable tackers. Further fixation can be performed with sutures but one should take care not to break needles in the relative inflexible promotory. The needle should be advanced millimeter by millimeter, following the curve of the needle. After rectal fixation, the dorsal vaginal wall can be fixed to the mesh with two lateral sutures at the vaginal top. No traction should be applied. Finally the peritoneum is closed over the ventral aspect of the mesh with an absorbable running suture; self-locking wires are ideal for this task (Figure 6). 

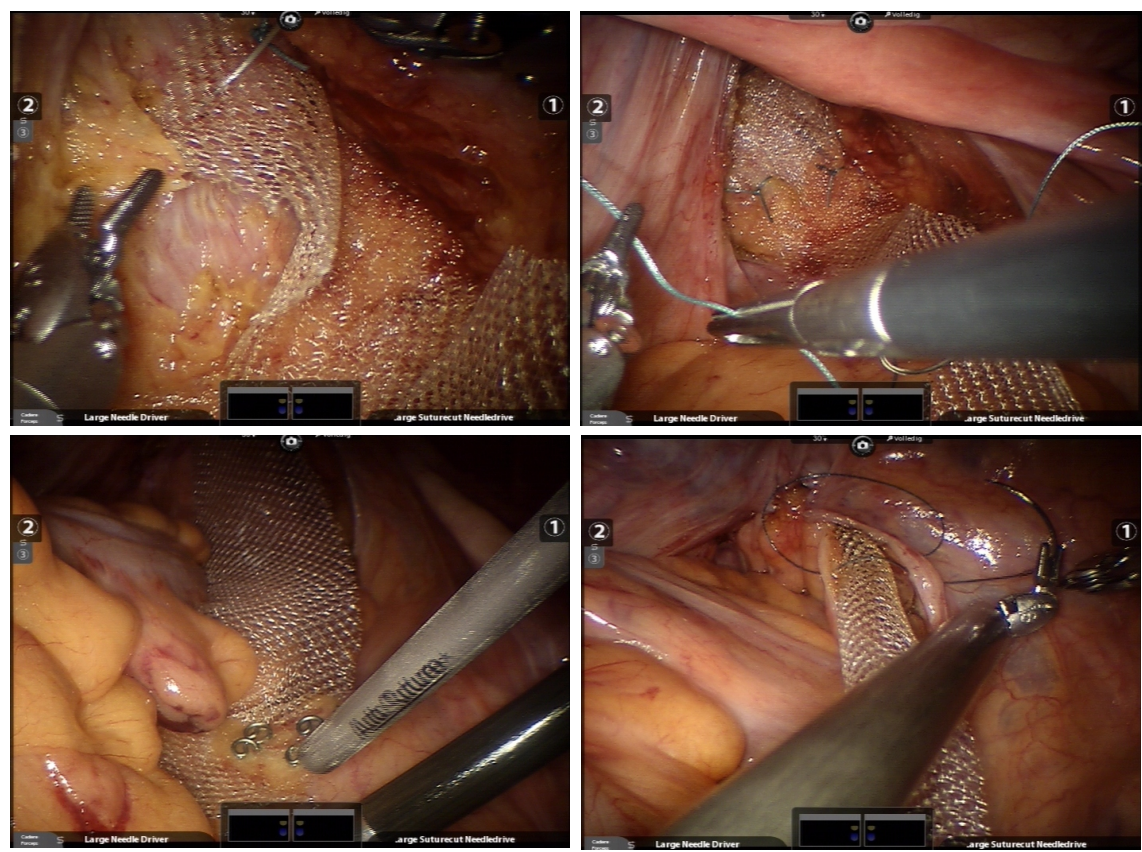

Figure 6. Suturing and tacking in robot-assisted ventral rectopexy.

\section{Why USE ROBOTIC TECHNOLOGY?}

Numerous surgical techniques for the treatment of rectal prolapse have been described over the last few centuries. In recent years, laparoscopic rectopexy procedures gained popularity due to good functional results, low morbidity and a low recurrence rate. Nowadays, laparoscopic ventral rectopexy is the treatment choice in Europe. Robotic assistance is used for several reasons. It allows perfect camera control in a narrow space with steep angles and difficult visualization of the key structures. The surgeon can always focus on the region of interest and the risk of lens blurring by touching tissue is minimized. The 3-D view is most supportive in discriminating the right dissection plane and in avoiding small vessels in the area between rectum and vagina. A stable though versatile third arm always provides the most optimal presentation of the area of interest. Suturing deep down in the pelvis is very difficult by standard techniques and may require the use of knotpushers. The dextrous robotic technology brings suturing to a new level. This improves quality and certainly saves time in these procedures. Surgeons tend to place more sutures using the robot because of the ease of manipulation. Finally, the surgeon's ergonomics improve drastically in pelvic surgery when using a robot and this may prove to be of great value for those who perform this type of surgery on a large scale. 


\section{REFERENCES}

1. Wu, JS. Rectal prolapse: a historical perspective. Curr Probl Surg, 2009, 46(8), 602-716.

2. Wijffels, NA; Collinson, R; Cunningham, C; Lindsey, I. WHAT IS THE NATURAL HISTORY OF INTERNAL RECTAL PROLAPSE ? Colorectal Dis, 2009.

3. Elneil, S. Complex pelvic floor failure and associated problems. Best Pract Res Clin Gastroenterol, 2009, 23(4), 555-73.

4. Madiba, TE; Baig, MK; Wexner, SD. Surgical management of rectal prolapse. Arch Surg, 2005, $140(1), 63-73$.

5. Formijne, Jonkers, HA; Draaisma, WA; Wexner, SD; et al. Evaluation and surgical treatment of rectal prolapse: An international survey. Colorectal Dis 2012.

6. Kuijpers, HC. Treatment of complete rectal prolapse: to narrow, to wrap, to suspend, to fix, to encircle, to plicate or to resect? World J Surg, 1992, 16(5), 826-30.

7. D'Hoore, A; Cadoni, R; Penninckx, F. Long-term outcome of laparoscopic ventral rectopexy for total rectal prolapse. Br J Surg, 2004, 91(11), 1500-5.

8. Frykman, HM; Goldberg, SM. The surgical treatment of rectal procidentia. Surg Gynecol Obstet, 1969, 129(6), 1225-30.

9. Laubert, T; Kleemann, M; Schorcht, A; et al. Laparoscopic resection rectopexy for rectal prolapse: a single-center study during 16 years. Surg Endosc, 2010.

10. Ayav, A; Bresler, L; Hubert, J; Brunaud, L; Boissel, P. Robotic-assisted pelvic organ prolapse surgery. Surg Endosc, 2005, 19(9), 1200-3.

11. Munz, Y; Moorthy, K; Kudchadkar, R; et al. Robotic assisted rectopexy. Am J Surg, 2004, 187(1), 88-92.

12. Heemskerk, J; de, Hoog, DE; van, Gemert, WG; et al. Robot-assisted vs. conventional laparoscopic rectopexy for rectal prolapse: a comparative study on costs and time. Dis Colon Rectum, 2007, 50(11), 1825-30.

13. D’Hoore, A; Penninckx, F. Laparoscopic ventral recto(colpo)pexy for rectal prolapse: surgical technique and outcome for 109 patients. Surg Endosc, 2006, 20(12), 1919-23.

14. Collinson, R; Cunningham, C; D'Costa, H; Lindsey, I. Rectal intussusception and unexplained faecal incontinence: findings of a proctographic study. Colorectal Dis, 2009, 11(1), 77-83.

15. Weld, KJ; Arzola, J; Montiglio, C; Bush, AC; Cespedes, RD. Lapra-Ty holding strength and slippage with various suture types and sizes. Urology, 2008, 71(1), 32-5.

16. Formijne, Jonkers, HA; van de Haar, HJ; Draaisma, WA; et al. The optimal strategy for proximal mesh fixation during laparoscopic ventral rectopexy for rectal prolapse: an ex vivo study. Surg Endosc, 2012. 


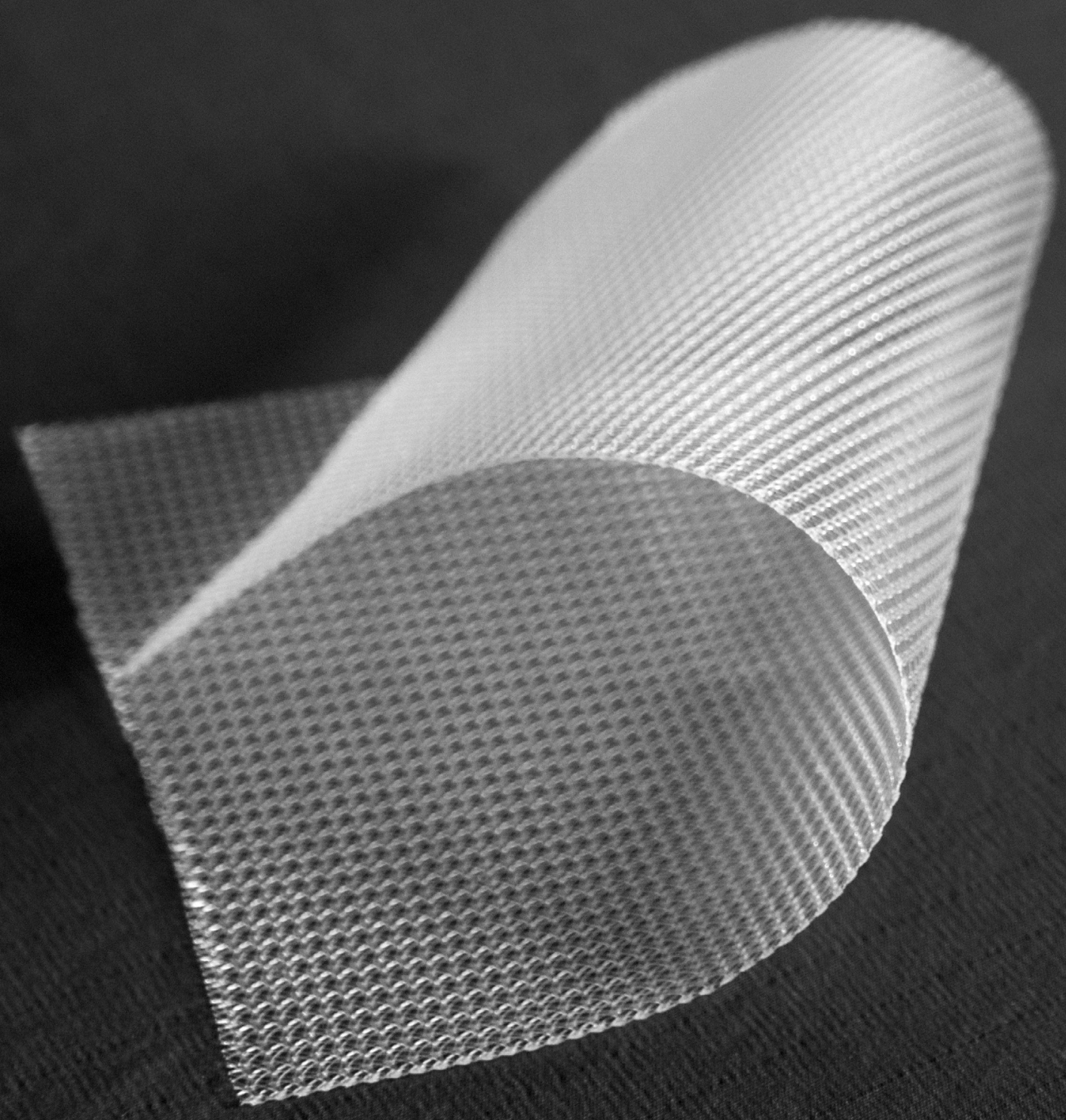




\section{Chapter 9}

\section{Learning curve for robot-assisted laparoscopic ventral rectopexy}


ABSTRACT

Introduction

Laparoscopic ventral rectopexy is nowadays favored for the treatment of rectal prolapse in Europe. The use of a robotic system during this procedure may support complex dissection up to the pelvic floor and allow precise suturing in a narrow, distance space. The aim of this study was to study the learning curve of robot-assisted laparoscopic ventral rectopexy (RALVR) for an experienced pelvic floor surgeon.

\section{Methods}

The four-armed da Vinci ${ }^{\circledR}$ Si HD surgical system (Intuitive Surgical Inc., Sunnyvale, CA) was used for this study. Patient demographics, periand postoperative complications, total operation time (TOT) and surgeon console time( SCT) were prospectively gathered between February 2011 (first RALVR) and July 2012. Accomplishment of the learning curve was determined using plots. Thereafter, SCT, TOT and complications before and after achievement of the learning curve were analyzed and compared.

\section{Results}

Thirty-five patients (32 females) with a mean age of 58.8 (range 26-87;SD \pm 14.7 ) were included. The surgeons' learning curve was reached after ten procedures (mean SCT before: 70.9 min, after: $53.3 \mathrm{~min}, \mathrm{P}=0.047$ ). The TOT reached a plateau after 20 procedures (mean TOT before: 114.2 min., after: 85.5 min., $P=0.06$ ). No intraoperative complications occurred. A total postoperative morbidity rate of $17 \%(n=6)$ was found, equally distributed over the consecutive cases.

\section{Conclusion}

Experienced pelvic floor surgeons can become proficient in RALVR after ten operations. The learning curve for the total procedure is twenty procedures when working with an experienced OR team. In addition, RALVR appears to be a safe alternative to conventional laparoscopic ventral rectopexy in the initial phase. 


\section{INTRODUCTION}

Prolapse of organs of the pelvic floor (POP) is a common condition in the western world, predominantly occurring in multiparous women above the age of 50. For females, the lifetime risk for developing POP is 30-50\%.[1] The rectum is often involved in this multi-organ problem. This results in (symptomatic) rectoceles, internal and external rectal prolapses. Affected patients usually suffer from fecal incontinence, obstructed defecation syndrome or a combination of both.[2]

One of the laparoscopic techniques for the treatment of rectal prolapse (RP) and large symptomatic rectoceles is laparoscopic ventral rectopexy (LVR). This mesh rectopexy technique proofed to be effective in terms of functional results in large cohorts of patients.[3-8] As these results are accompanied by a low morbidity rate this surgical technique has become accepted by many surgeons. This was illustrated by a recent survey that showed that LVR is the treatment of choice for rectal prolapse by the majority of surgeons throughout Europe.[9] LVR involves complex maneuvers like dissection and suturing deep in the pelvis.[5] Therefore, the use of a robotic system can be of technical support during this procedure. Complex surgical technology requires learning and adaptation. Information on the extend of this learning process is helpful during the implementation of robotic surgery in pelvic floor centers as the availability of robotic systems is increasing. Therefore the aim of the current study was to review the operation time and complications during the initial phase of RALVR in our center.

\section{Patients and methods}

\section{Study design and surgeon}

This study was carried out in a large teaching hospital in the Netherlands (Meander Medical Center, Amersfoort). It comprised a prospective cohort study to investigate the learning curve of an experienced laparoscopic colorectal surgeon (EC) for RALVR. Prior to this study, EC had no experience with robot-assisted procedures and performed about 250 conventional LVR procedures. The OR team supporting the surgeon was involved in more that 20 small pelvis interventions before the start of this study.

\section{Robotic surgical system}

The four-armed da Vinci® ${ }^{\circledR}$ Si HD surgical system (Intuitive Surgical Inc., Sunnyvale, CA) was used for this study.

\section{Ethics}

This study was undertaken in accordance with the ethical standards of the Declaration of Helsinki (1975), as revised in 1983. 


\section{Patients and data}

All patients who underwent RALVR were prospectively entered in a database. All relevant patient characteristics and demographics were collected prior to surgery. During surgery, total operation time (TOT), surgeon console time (SCT), intra-operative complications and total blood loss were registered using specially developed registration forms. For TOT the time from incision until closure of the incisions was used. Total hospital stay, complications and readmissions up to 90 days after surgery were recorded in the postoperative phase. Complications were classified according to the Clavien-Dindo classification.[10] Exclusion criteria for this study were a history of rectal surgery and/or pelvic radiation therapy.

\section{Procedure}

RALVR was performed under general anesthesia. The routine preparations comprised of a single dose of prophylactic intravenous antibiotics (servazolin 1000 $\mathrm{mg}$. and $500 \mathrm{mg}$. of metronidazole) administered 15 minutes prior to incision and a urinary catheter. With the use of adaptive leg supports with swing stirrups a steep French Trendelenburg position was achieved. The four-armed robotic cart was subsequently positioned at the left side of the patient at the level of the knee. The camera trocar was introduced through the upper margin of the umbilicus and another three $8-\mathrm{mm}$ robotic ports were subsequently placed. An additional $11-\mathrm{mm}$ or $12-\mathrm{mm}$ trocar was introduced next to the camera trocar and was used for assistance. After retraction of the rectosigmoid junction to the left, a peritoneal incision was made over the right side of the sacral promontory and extended to an inverted J-form along the rectum. The rectovaginal septum (in female patients) or Denonvillier's fascia (in male patients) was then opened. This was performed all the way to the level of the anal sphincter and pelvic floor muscles. Next, a $3 \mathrm{~cm} \times 15 \mathrm{~cm}$ strip of polypropylene mesh (Prolene ${ }^{\circledR}$, Johnson \& Johnson, USA) was fixed to the ventral aspect of the distal end of the rectum using six individual sutures. The proximal end of the mesh was then secured to the sacral promontory using a Tack device (Protack III ${ }^{\circledR}$, Covidien, USA). The posterior vaginal fornix was elevated and sutured to the anterior aspect of the mesh in female patients thereby closing the rectovaginal septum. Finally, the peritoneum was approximated in order to cover the mesh. This was done using a running absorbable suture. The fascia (at the camera port and assistant port) and the skin were closed in a regular fashion.

\section{Admission, post-operative care and discharge criteria}

All patients were admitted on the day of surgery. Postoperatively, all patients received a normal diet and were encouraged to mobilize within 24 hours. The urinary catheter was removed within 24 hours. All patients received a daily sub- 
cutaneous dose of low molecular heparin $(0.3 \mathrm{cc}$ or $0.6 \mathrm{cc}$ depending on bodyweight) during the hospitalization period. A polyethylene glycol laxative (Movicolon $^{\circledR}$ ) was started in all patients. Patients were discharged after tolerance of full diet, passage of stool, mobilizing properly and sufficient analgesia with oral analgesics. Evaluation at the outpatient clinic was routinely performed between 6 and 8 weeks after surgery.

\section{Statistical analysis}

Statistical analysis was performed using SPSS 20 (SPSS Inc. Chicago, IL, USA). Data are presented as mean and range. Statistical analysis was performed after creating groups of five consecutive procedures in order to correct outcomes for single outlying cases. The mean TOT and mean SCT of these five surgeries were used for comparison. These times were plotted against the operation number to determine the plateau phase from the graph. This plateau indicates a steady state in TOT and SCT.

Subsequently, two groups were defined: group one consisted of procedures during the learning period. The second group consisted of surgeries after completion of the learning curve. For TOT and SCT, the groups were analyzed for equality of variances using Levene's test. Subsequently, a t-test for equality of means was done, taking the result of Levene's test into account. A p-value of 0.05 was considered significant.

\section{RESULTS}

\section{Patients and indications for surgery}

Thirty-five patients ( 3 males; 32 females) with a mean age of 58.8 years (range 26-87; SD \pm 14.7 ) were included. Indications for surgery were large symptomatic rectocele or internal rectal prolapse $(n=14,40 \%)$, large symptomatic rectocele or internal rectal prolapse with a concomitant enterocele $(n=14,40 \%)$ and external rectal prolapse $(n=7,20 \%)$. Further patient demographics are presented in Table 1. 
Table 1. Baseline, admission and complication rate characteristics.

\begin{tabular}{lc}
\hline Mean age in years (range; SD) & RALVR cohort (N=35) \\
Sex & $55.8(26-87 ; \pm 14.7)$ \\
$\quad$ female (\%) & $33(94.3 \%)$ \\
$\quad$ male (\%) & $2(5.7 \%)$ \\
ASA classification (\%) & ASA $1: 11(31.4 \%)$ \\
& ASA 2: $19(54.3 \%)$ \\
& ASA 3: $4(11.4 \%)$ \\
Prior abdominal/pelvic surgery & ASA 4: $1(2.9 \%)$ \\
$\quad$ Of which hysterectomy & $8(22.9 \%)$ \\
Mean admission in days (range; SD) & $4(11.4 \%)$ \\
Complications during surgery & $3.3(2-11 ; \pm 1.7)$ \\
Post-operative complications (\%) & $0(0 \%)$ \\
(see also table 3) & $6(17.1 \%)$ \\
\hline
\end{tabular}

\section{Operation times and learning curve}

Figure 1 shows the TOT and SCT analyzed per group. The plateau phase (learning curve) for SCT was established after 10 LVR procedures. For TOT a steady state was reached after a total of 20 procedures.

Group 1 and 2 were statistically analyzed and compared with regard to SCT and TOT. The mean SCT was significantly shorter after completion of the learning curve (53.3 min. versus 70.9 min. respectively, $\mathrm{P}=0.047$ ). The TOT after completion of the learning curve was also shorter compared to prior of achievement of the learning curve (85.5 min. versus $114.2 \mathrm{~min}$., respectively). Statistical significance was however not reached for this latter comparison $(P=0.06)$. Statistical analyses including Levene's tests are presented in Table 2.

\section{Admission, complications, blood loss and readmissions}

The mean hospital stay for the total cohort was 3.3 days (range: $2-11, \mathrm{SD} \pm 1.7$ ). No intra-operative complications occurred in this study and there were no conversions to either conventional laparoscopy or laparotomy. Total blood loss was less than $200 \mathrm{ml}$ for all cases.

Six post-operative complications occurred (17\%). These consisted of Clavien-Dindo class I ( $n=4,11 \%)$, CD class II $(n=1,3 \%)$ and CD class III $(n=1,3 \%)$. An overview of all post-operative complications is presented in Table 3. 


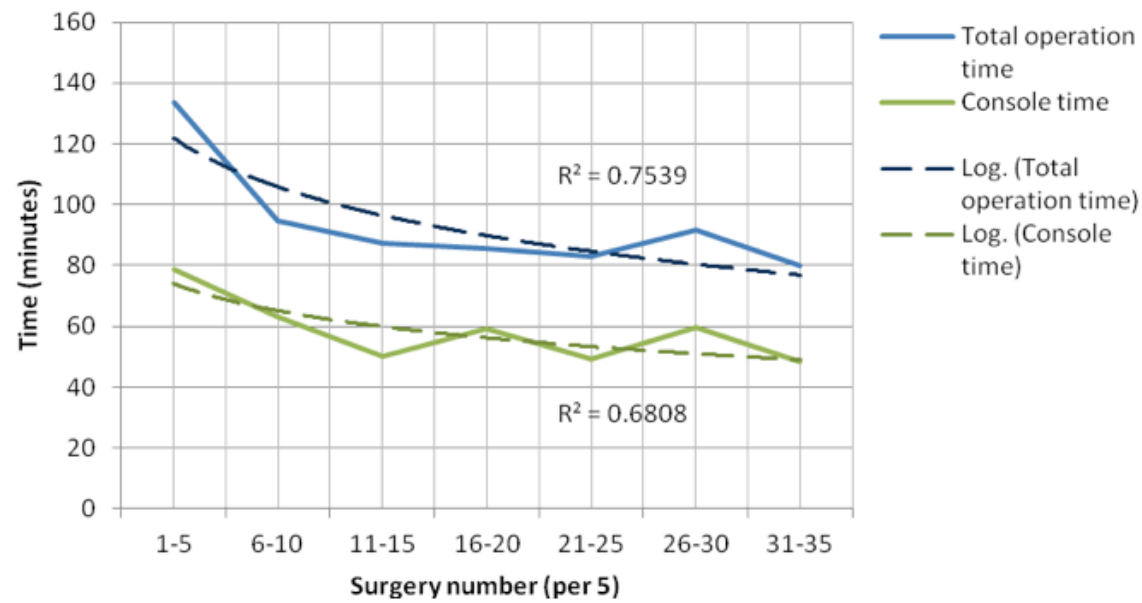

Figure 1. Recorded TOT and SCT in minutes per group of 5 consecutive cases. The dotted line represents the trend.

Table 2. Results ( $p$-values) of the statistical analyses.

\begin{tabular}{lcc} 
& p-value Levene's test & p-value t-test \\
\hline Total surgery time (TOT) & 0.086 & 0.061 \\
Console time (SCT) & 0.207 & 0.047 \\
\hline
\end{tabular}

Table 3. Overview of all complications including Clavien-Dindo (CD) classification.

\begin{tabular}{llcc}
\hline Case no. & Post-operative complications \& management & $\mathbf{n = ( \% )}$ & CD Class. \\
\hline 4,24 & Unaccountable pain treated with oral analgesia & $2(5.7 \%)$ & 1 \\
6 & Mechanical ileus (caused by adhesions): laparotomy & $1(2.9 \%)$ & 3 \\
& two weeks after RALVR & & \\
17 & $\begin{array}{l}\text { Readmission because of nausea and vomiting: conser- } \\
\text { vative treatment with antiemetics }\end{array}$ & $1(2.9 \%)$ & 1 \\
8 & Electrolyte disturbance treated with IV supplements & $1(2.9 \%)$ & 1 \\
22 & Urinary tract infection treated with oral antibiotics & $1(2.9 \%)$ & 1 \\
\hline & Total & $\mathbf{6 ( 1 7 . 1 \% )}$ & \\
\hline
\end{tabular}

There was one readmission (3\%) 13 days after surgery because of a mechanical ileus. This was successfully treated with a laparotomy and adhesiolysis on the next day. The patient was discharged after 5 days. This complication is also registered in Table $\mathbf{3}$ (case no. 6). 
There was no significant difference between the number of complications before and after completion of the learning curve $(P>0.05)$.

\section{Discussion}

Ventral mesh rectopexy was originally described as an open procedure but became popular after introduction of endoscopic techniques. [6;9;11] The use of a robot surgical system is the next logical step for ventral rectopexy as the robot is designed to offer a better view and dexterity in small spaces. In the current study it was found that the learning curve for RALVR is 20 procedures for the entire procedure and just 10 procedures for the console phase. After completion of the learning curve, the mean SCT was 53.3 minutes.

Currently available literature on RALVR and its learning curve is limited. The only described learning curve for robot-assisted rectopexy was completed after 18 procedures. In that study, several different techniques including RALVR were performed by three different surgeons. The results were combined in one learning curve and are therefore not representative to determine the exact learning curve for RALVR. [12] The operation time for RALVR has been reported in several studies, and varied between a mean of 159 minutes and 223 minutes. [12;13] Operation times in our study were short compared to these reports, even in the beginning of the learning curve. This was probably caused by the extensive experience of the surgeon with conventional LVR prior to this study (> 250 procedures). Additionally, the dedicated operation room staff was trained in robot-assisted surgery prior to this study, resulting in adequate assistance during RALVR. Moreover, it is not clear whether the stated operation time in previous studies is the time from incision to closure, or that it represents the total time of anesthesia or perhaps even the total time of the patients in the operation suite. Ventral rectopexy is known to be a safe procedure associated with low complication rates. During conventional laparoscopic ventral rectopexy, complication rates vary between $4.6 \%$ and $21 \%$ and usually consist of minor complications. $[3 ; 4 ; 6 ; 8]$ Reported morbidity rates in previous robot-assisted series are around $2-10 \%$. [12-14] The current study found a complication rate of $17,1 \%$. This is rather high compared to literature. However, all but one of these complications were low grade complications (CD grade I) and the vast majority was treated conservative using medications like antalgics, anti-emetics and electrolytes. The results of this study therefore suggest that RALVR is a safe procedure even in the initial phase of the learning curve of an otherwise experienced pelvic floor surgeon.

Robot-assisted surgery is associated with significantly higher costs compared to conventional laparoscopy. In a previous study, the costs for RALVR were calculated to be $€ 3.672,84$ versus $€ 3.115,55$ for LVR. These costs did not include 
the high costs for purchase and maintenance of the da Vinci ${ }^{\circledR}$ system. [15] Until now these costs are not justified by demonstrating a shorter operation time or less complications for RALVR compared to LVR, and chances that this will be the case in the near future are slim. The use of robotics should therefore lead to better functional results at long term.

Subjectively, the magnificent three-dimensional view of the robot is supportive in discriminating the correct dissection plane and in avoiding small vessels in the area between rectum and vagina. Furthermore, suturing the mesh to the ventral aspect of the rectal wall deep in the pelvis is brought to a higher level with the robot compared to the ergonomic inefficiencies that have to be overcome during conventional LVR. Superior view and ergonomics might result in a better and deeper position of the mesh all the way down to the pelvic floor. Whether this correspondingly leads to better functional outcomes regarding ODS and incontinence is something that will be addressed in large future trials. In conclusion, the learning curve for RALVR for an experienced surgeon at the console is reached after 10 procedures and after 20 procedures for the complete procedure, when supported by an experienced OR team. 


\section{REFERENCE LIST}

1 Nygaard I, Bradley C, Brandt D: Pelvic organ prolapse in older women: prevalence and risk factors. Obstet Gynecol 2004;104:489-497.

2 Elneil S: Complex pelvic floor failure and associated problems. Best Pract Res Clin Gastroenterol 2009;23:555-573.

3 Boons P, Collinson R, Cunningham C, Lindsey I: Laparoscopic ventral rectopexy for external rectal prolapse improves constipation and avoids de novo constipation. Colorectal Dis 2010;12:526-532.

4 Collinson R, Wijffels N, Cunningham C, Lindsey I: Laparoscopic ventral rectopexy for internal rectal prolapse: short-term functional results. Colorectal Dis 2010;12:97-104.

5 D’hoore A, Penninckx F: Laparoscopic ventral recto(colpo)pexy for rectal prolapse: surgical technique and outcome for 109 patients. Surg Endosc 2006;20:1919-1923.

6 Formijne Jonkers HA, Poierrie N, Draaisma WA, Broeders IA, Consten EC: Laparoscopic ventral rectopexy for rectal prolapse and symptomatic rectocele: an analysis of 245 consecutive patients. Colorectal Dis 2013;15:695-699.

7 Wijffels N, Cunningham C, Lindsey I: Laparoscopic ventral rectopexy for obstructed defecation syndrome. Surg Endosc 2009;23:452.

8 Wijffels N, Cunningham C, Dixon A, Greenslade G, Lindsey I: Laparoscopic ventral rectopexy for external rectal prolapse is safe and effective in the elderly. Does this make perineal procedures obsolete? Colorectal Dis 2011;13:561-566.

9 Formijne Jonkers HA, Draaisma WA, Wexner SD, Broeders IA, Bemelman WA, Lindsey I, Consten EC: Evaluation and surgical treatment of rectal prolapse: an international survey. Colorectal Dis 2013;15:115-119.

10 Dindo D, Demartines N, Clavien PA: Classification of surgical complications: a new proposal with evaluation in a cohort of 6336 patients and results of a survey. Ann Surg 2004;240:205-213.

11 Silvis R, Gooszen HG, van EA, de Kruif AT, Janssen LW: Abdominal rectovaginopexy: modified technique to treat constipation. Dis Colon Rectum 1999;42:82-88.

12 Perrenot C, Germain A, Scherrer ML, Ayav A, Brunaud L, Bresler L: Long-term outcomes of robotassisted laparoscopic rectopexy for rectal prolapse. Dis Colon Rectum 2013;56:909-914.

13 Mantoo S, Podevin J, Regenet N, Rigaud J, Lehur PA, Meurette G: Is robotic-assisted ventral mesh rectopexy superior to laparoscopic ventral mesh rectopexy in the management of obstructed defaecation? Colorectal Dis 2013;15:e469-e475.

14 Makela-Kaikkonen J, Rautio T, Klintrup K, Takala H, Vierimaa M, Ohtonen P, Makela J: Roboticassisted and laparoscopic ventral rectopexy in the treatment of rectal prolapse: a matched-pairs study of operative details and complications. Tech Coloproctol 2014;18:151-155.

15 Heemskerk J, de Hoog DE, van Gemert WG, Baeten CG, Greve JW, Bouvy ND: Robot-assisted vs. conventional laparoscopic rectopexy for rectal prolapse: a comparative study on costs and time. Dis Colon Rectum 2007;50:1825-1830. 


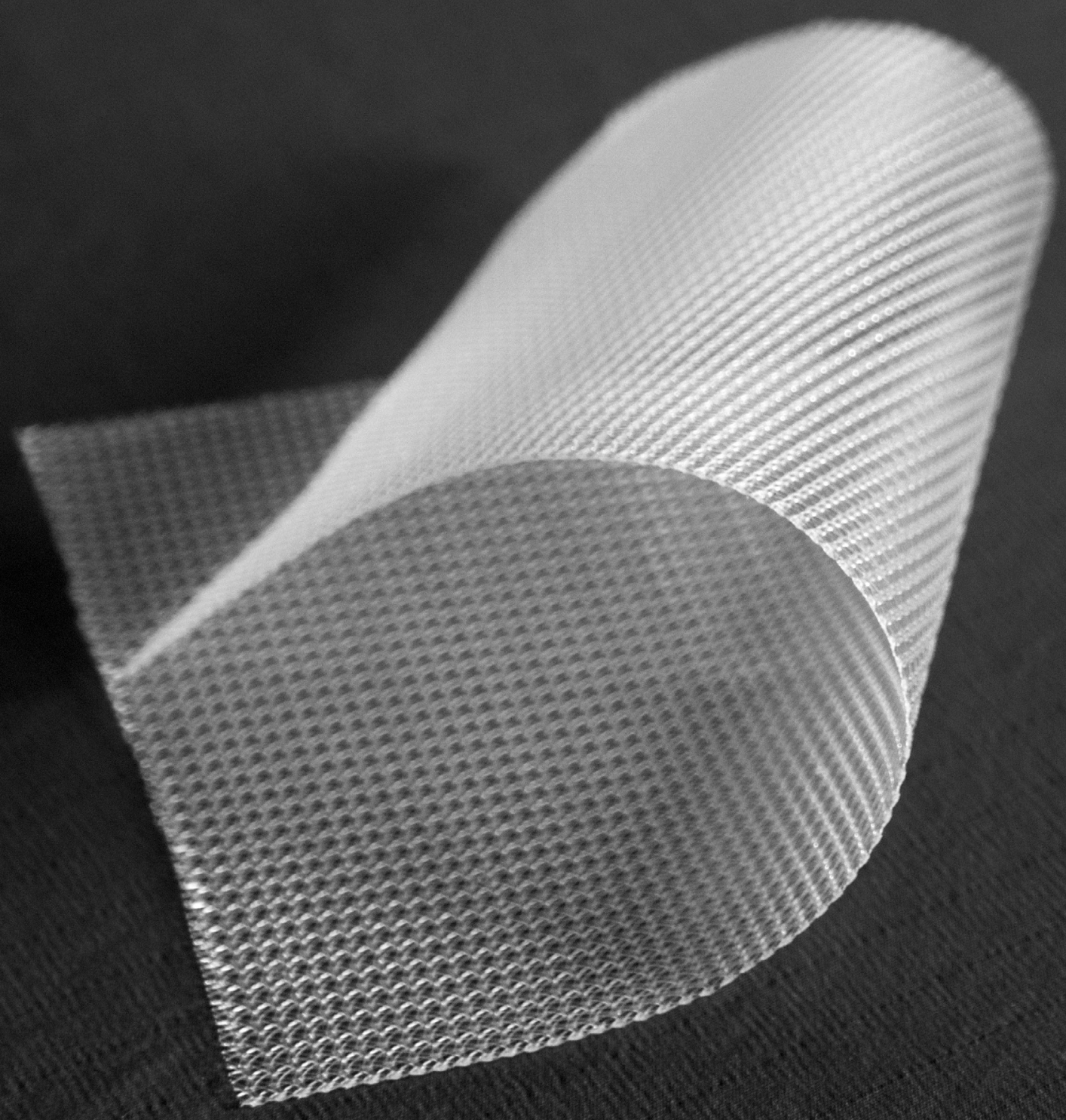




\section{Chapter 10}

\section{Robot-Assisted Laparoscopic Ventral Rectopexy for rectal prolapse:}

analysis of a large cohort of patients 
Aim

This study aimed to determine the complications and functional results of Robot-Assisted Laparoscopic Ventral Rectopexy (RALVR) for rectal prolapse (RP) and symptomatic rectoceles in a large cohort of patients.

\section{Methods}

All patients treated between 07-2010 and 04-2013 were identified. Relevant patient characteristics were retrospectively gathered. Main endpoints were functional complaints (incontinence and constipation/obstructed defecation syndrome (ODS)), morbidity and mortality.

\section{Results}

146 patients (141 female, 5 male) were included for analysis. Mean age was 59.8 years at the time of surgery (range 25-96, SD \pm 13.4 ). Indications for RALVR were: external RP $(n=23)$, internal RP or symptomatic rectocele $(n=79)$ or a combination of symptomatic rectocele and enterocele $(n=44)$. Mean operation time was $87.2 \mathrm{~min}$ - utes (range 55.0 - 218.2 SD \pm 18.8 ). The total morbidity rate was $8.2 \%$ and consisted in the vast majority of patients (75\%) of low-grade complications as determided by the Clavien-Dindo scoring system. Mortality after surgery was $0.7 \%$ ( 1 case). The incidence of symptoms of incontinence and constipation/ODS both significantly decreased after RALVR compared to the preoperative rate (8.9\% versus $54.1 \%$ for incontinence and $15.1 \%$ versus $58.2 \%$ for constipation, respectively (both $\mathrm{P}<0.01$ )). This included 1 case $(0.7 \%)$ of incontinence de novo and 2 cases (1.4\%) of constipation de novo after surgery.

\section{Conclusion}

RALVR proved to be safe and effective for the treatment of RP and large symptomatic rectoceles in the largest cohort of patients published up-todate. Future trials are needed to determine whether results are superior to conventional laparoscopic ventral rectopexy. 


\section{INTRODUCTION}

Incontinence, constipation and the inability to empty the rectum during defecation (the Obstructed Defecation Syndrome (ODS)) are common functional complaints of the gastro-intestinal (GI) tract.[1-3] Although there is a wide array of disorders that can induce these symptoms, the cause is often found in anatomical disorders of the pelvic floor like rectoceles and complete prolapses. These symptoms are frequently found in females of middle or older age and in combination with prolapses of other organs of the pelvic floor.[4]

The initial treatment of rectal prolapse (RP) should be conservative. Examples of conservative treatment are dietary changes, the prescription of bulking agents and biofeedback therapy of the pelvic floor. The only definite treatment, however, is surgery. [5;6] Conventional laparoscopic ventral rectopexy (LVR) seems to be the treatment of choice for RP for the majority of surgeons in Europe and an increasing number of surgeons in the US. [7;8] Growing evidence on improved functional outcomes after LVR for the treatment of external RP has attributed to the cause of this growing popularity. In recent years the indication of LVR became wider as it also proved to be successful for the treatment of large symptomatic rectoceles and internal rectal prolapse. [9;10]

Dissection of the rectovaginal septum down to the pelvic floor is one of the essential steps of LVR. Subsequently, a mesh is sutured to the most distal portion the rectal wall. Frequently these two steps cause ergonomic struggles for the surgeon during surgery. Some recent studies therefore described the use of a robotic system during LVR as these high-tech systems enhance visualization, dexterity and precision in confined spaces. [11-13] It is however unknown whether the use of a robotic system also leads to better patient related results. The aim of the current study was therefore to describe a large cohort of patients treated with Robot-Assisted Laparoscopic Ventral Rectopexy (RALVR) with special focus on functional results and complications.

\section{Methods}

\section{Study design}

Retrospective analysis of all consecutive patients who underwent RALVR for internal or external RP and symptomatic rectocele (with or without enterocele) between 07-2010 and 04-2013. This study was carried out in a large teaching hospital in the Netherlands (Meander Medical Center, Amersfoort). All patients were operated by one of three experienced pelvic floor surgeons. Patients who underwent primary surgery were included for analysis. Demographics, medical history, operation details, morbidity, mortality and follow-up details of patients were collected from their medical records and gathered into a database. 


\section{Ethics}

This study was undertaken in accordance with the ethical standards of the Declaration of Helsinki (1975), as revised in 1983.

\section{Pre-operative evaluation and indications for surgery}

Surgery was performed after an extensive work-up. This included a detailed history comprising standardized questionnaires, physical examination and radiological examination of the pelvic floor (dynamic Magnetic Resonance Imaging (D-MRI)). In selected cases an additional evacuation proctography was performed, for example in case of an inconclusive D-MRI. Both D-MRI and evacuation proctography were performed with intra-vaginal contrast and intra-rectal contrast enema. A colonic transit study was performed if estimated helpful in the diagnosis of constipation/ODS.

Subsequently, all patients were discussed in a multidisciplinary meeting attended by dedicated gynecologists, urologists, radiologists, pelvic floor physical therapists and pelvic floor surgeons.

Indications for surgery were an external RP (Oxford classification grade V) as well as a history of incontinence or constipation/ODS in combination with an Oxford grade III or IV internal RP. Surgery was also performed in patients with incontinence and/or constipation/ODS in combination with a large anterior rectocele. This was defined as $>2 \mathrm{~cm}$. bulging of the anterior rectal wall during physical examination or seen on radiological imaging studies. Pelvic floor biofeedback therapy was started if estimated useful (e.g. in case of a concomitant descensus of the pelvic floor).

\section{Surgical technique}

The procedure was performed under general anesthesia. Prophylactic intravenous antibiotics (servazolin $1000 \mathrm{mg}$. and $500 \mathrm{mg}$. of metronidazole) were administered 15 minutes prior to incision and a urinary catheter was introduced in a standard fashion. The four-armed da Vinci ${ }^{\circledR}$ Si HD surgical system (Intuitive Surgical Inc., Sunnyvale, CA) was used during surgery in this study. The robotic cart was positioned at the left side of the patient. The camera trocar was then introduced through an incision made at the upper margin of the umbilicus. Three 8 -mm robotic ports were used for dissection and suturing during this procedure. An additional port was placed between the camera trocar and the robotic trocar and was used for assistance. Subsequently, the patient was positioned in a steep French Trendelenburg position using adaptive leg supports with swing stirrups.

The rectosigmoid junction was retracted to the left and a J-shaped peritoneal incision was made over the right side of the sacral promontory and extended along the rectum. The rectovaginal septum was than opened up to the level of 
the anal sphincter and pelvic floor muscles. Subsequently, a $3 \mathrm{~cm} \times 15 \mathrm{~cm}$ strip of polypropylene was fixed to the ventral aspect of the distal rectum using six individual sutures. The proximal end of the mesh was fixed to the sacral promontory using a Tack device (Protack III ${ }^{\circledR}$, Covidien, USA). In female patients the rectovaginal septum was closed by elevating the posterior vaginal fornix and subsequently suturing it to the anterior aspect of the mesh. The peritoneum was approximated using a running resorbable suture. Fascia and skin were closed in a standard manner.

\section{Outcome parameters}

Symptoms of incontinence and constipation / ODS before and after surgery were the main outcome parameters of this study. The Rome II criteria were used for routinely pre- and postoperative assessment of Constipation and ODS. Fecal incontinence was defined as the involuntarily loss of solid or liquid stool, once or more, during the last month. This included symptoms of anal soiling. Intra-operative and postoperative complications up to 30 days after surgery were registered. The Clavien-Dindo Classification was used to score postoperative complications (CD).[14]

\section{Statistical Analysis}

Statistical analysis was performed using Statistical Package for the Social Science, version 19.0 (SPSS Inc., Chicago, IL, USA). Patient data are presented as percentage or as mean \pm S.D and range for all numerical variables.

For the descriptive analyses, McNemar tests were used to evaluate differences in percentages. $P$ values $<0.05$ were considered significant.

\section{RESULTS}

\section{Patients and indications for surgery}

A total of 146 patients ( 141 females, 5 males) were included for analysis. Mean age was 59.8 years (range $25-96, \mathrm{SD} \pm 13.4$ ) at the moment of surgery. The indications for surgery were an external RP in 23 patients (15.8\%), internal RP or symptomatic rectocele $(n=79,54.1 \%)$ and internal RP or symptomatic rectocele plus an enterocele in 44 patients (30.1\%). Further baseline characteristics can be found in table 1.

\section{Surgery and complications}

The mean total operation time was 87.2 minutes (range 55.0 - 218.2 minutes, SD \pm 18.8 ). There were no conversions to either conventional laparoscopy or laparotomy during surgery. Intra-operative complications did not occur and the estimated blood loss was less than $200 \mathrm{ml}$. for all cases. There were $12(8.2 \%)$ postoperative complications, all in different cases. The majority of 
Table 1. Baseline and admission details.

\begin{tabular}{ll}
\hline & RALVR cohort (N=146) \\
\hline Mean age in years (range; SD) & $59.8(25-96, \mathrm{SD} \pm 13.4)$ \\
$\quad$ female (\%) & $141(96.6 \%)$ \\
$\quad$ male (\%) & $5(3.4 \%)$ \\
ASA classification (\%) & ASA 1: $53(36.3 \%)$ \\
& ASA 2: $86(58.9 \%)$ \\
& ASA 3: $7(4.8 \%)$ \\
Prior abdominal/pelvic surgery & $8(22.9 \%)$ \\
$\quad$ Of which hysterectomy & $54(37.0 \%)$ \\
Mean admission in days (range; SD) & $3.28(2-12 ; \pm 1.4)$ \\
Complications during surgery & $0(0 \%)$ \\
Post-operative complications (\%) & $12(8.2 \%)$ \\
(see also table 2) & \\
\hline
\end{tabular}

these complications were $C D$ grade $I(n=6,4.1 \%)$ and $C D$ grade II complications $(n=3,2.1 \%)$. Two patients were operated because of postoperative ileus based on adhesions (CD grade $3 \mathrm{~b}, 1.4 \%$ ). In one patient a laparoscopic adhesiolysis was performed and the other subject underwent a laparotomy for adhesiolysis. One patient (female, 91 years old, external rectal prolapse, ASA class II) was readmitted three weeks after uncomplicated surgery and admission because of a severe septic shock based on bowel ischemia. Palliative care was started and the patient died (CD grade $5,0.7 \%$ ). An overview of all postoperative complications can be found in table 2 .

\section{Functional results: incontinence}

In general, $54.1 \%$ of the patients $(n=79)$ had complaints of incontinence in some degree prior to surgery. This rate significantly decreased to 8.9\% (13 patients, $\mathrm{P}<0.01$ ) after surgery. Sub-group analysis showed a significant reduction of incontinence after RALVR for all indications. The results per indication are presented in table 3.

In total, one case of incontinence de novo after surgery was observed $(0.7 \%)$. 
Table 2. Overview of all complications and management according to the Clavien-Dindo classification (CD).

\begin{tabular}{lcc}
\hline Post-operative complications \& management & $\mathbf{n = ( \% )}$ & CD Class. \\
\hline Unaccountable upper abdominal pain treated with analgetics & $1(0.7 \%)$ & 1 \\
Electrolyte disturbance treated with IV supplements & $1(0.7 \%)$ & 1 \\
Anal blood loss till 5 days postoperative: conservative treatment & $1(0.7 \%)$ & 1 \\
Prolonged / paralytic ileus: conservative treatment with antiemetics & $2(1.4 \%)$ & 1 \\
Stable angina pectoris after surgery: conservative treatment & $1(0.7 \%)$ & 1 \\
Urinary tract infection treated with oral antibiotics & $1(0.7 \%)$ & 2 \\
Pneumonia treated with IV antibiotics & $1(0.7 \%)$ & 2 \\
Spondylodiscitis at point of proximal mesh fixation: IV antibiotics & $1(0.7 \%)$ & 2 \\
Mechanical ileus (caused by adhesions): surgical intervention & $2(1.4 \%)$ & $3 b$ \\
Bowel ischemia 3 weeks after surgery (see text): palliative care & $1(0.7 \%)$ & 5 \\
\hline Total & $\mathbf{1 2 ( 8 . 2 \% )}$ \\
\hline
\end{tabular}

\section{Functional results: Constipation/ODS}

The preoperative incidence of ODS/constipation in the total cohort of patients was was $58.2 \%$ (85 patients). This significantly decreased to 22 patients after RALVR (15.1\%, $\mathrm{P}<0.01)$. A similar significant reduction in constipation/ODS was observed for all indications, as can be appraised in table 3.

Two cases of ODS/constipation de novo (1.4\%) after surgery were observed in the total cohort of patients.

Table 3. Overview of functional results per indication

\begin{tabular}{lcccccc}
\hline Indication & $\begin{array}{c}\text { Pre-op. } \\
\text { incontinence }\end{array}$ & $\begin{array}{c}\text { Post-op. } \\
\text { incontinence }\end{array}$ & $\begin{array}{c}\text { P-value } \\
\text { Constipation/ } \\
\text { ODS }\end{array}$ & $\begin{array}{c}\text { Pre-op. } \\
\text { Constipation/ } \\
\text { ODS }\end{array}$ & \\
\hline $\begin{array}{l}\text { External rectal } \\
\text { prolapse }(\mathrm{n}=23)\end{array}$ & $12(52.2 \%)$ & $4(17.4 \%)$ & 0.01 & $8(34.8 \%)$ & $1(4.3 \%)$ & 0.01 \\
$\begin{array}{l}\text { Internal rectal } \\
\text { prolapse / rectocele } \\
(n=79)\end{array}$ & $36(54.4 \%)$ & $4(5.1 \%)$ & $<0.001$ & $51(64.5 \%)$ & $13(16.5 \%)$ & $<0.001$ \\
$\begin{array}{l}\text { Combination recto- } \\
\text { cele and enterocele } \\
(n=44)\end{array}$ & $24(54.5 \%)$ & $5(11.4 \%)$ & $<0.001$ & $20(59.1 \%)$ & $8(18.2 \%)$ & $<0.001$ \\
\hline
\end{tabular}




\section{Discussion}

The current study evaluated the largest cohort of patients up to date treated with RALVR for RP and rectoceles and is the first study to categorize complications and functional results of this procedure. It was found that the rate of incontinence and constipation/ODS decreases significantly after RALVR. This is accompanied with a low rate of complications.

Pelvic floor surgery, especially surgery for RP and rectoceles, is predominantly performed to decrease functional complaints like constipation/ODS and incontinence. In this cohort of patients the rate of both these symptoms is significantly lower after surgery when compared to the preoperative incidence: $8.9 \%$ vs. $54.1 \%$ for incontinence and $15.1 \%$ vs. $58.2 \%$ for constipation/ODS. Comparable results can be found in literature for conventional LVR: large cohorts of patients show significant reductions of symptoms but successful symptomatic outcome is never reported for all patients. $[7 ; 9 ; 15 ; 16]$ This is also seen in the few RALVR studies that are available today. $[12 ; 17]$ In general however, the functional results after RALVR seem to be better compared to LVR. We performed an identical analysis of the functional results of conventional LVR in our institution in 2011.[9] In direct comparison with the current study, persisting incontinence was seen in 14\% of patients after LVR versus $9 \%$ in the current study. ODS/constipation after surgery was observed in $19 \%$ after LVR compared to $15 \%$ after RALVR. Possibly, these better results are caused by enhanced visibility and maneuverability deep in the pelvis during RALVR compared to the conventional laparoscopic counterpart, resulting in a better distal position of the mesh, closer to the pelvic floor muscles. Whether this difference is significant has to be addressed in prospective, comparative studies. Furthermore, it is pivotal to determine the causes of failure of ventral rectopexy in future studies. As incontinence and constipation/ODS can be multifactorial, it is possible that patients with persisting functional complaints still have other factors that contribute to (or cause) these symptoms after rectopexy.[2;3] Examples could be a slow-transit colon or sphincter defects. Treatment algorithms should therefore be developed in order to offer a tailor-made approach to patients with a RP and minimize the risk of 'symptomatic failure'.

One of the explanations of the popularity of LVR nowadays is the low rate of complications. In large studies, complication rates vary between $4.6 \%$ and $21 \%$ and usually consist of minor complications. [7;9;18;19] Similar rates were found for RALVR.[11;12;17] Our study found a complication rate of $8.2 \%$ that consisted mostly of CD grade I and grade II complications. High-grade complications were seen in only 3 patients (2.1\%). Mortality after LVR and RALVR is extremely rare. The described case of fatal bowel ischemia in the current study occurred within 30 days after RALVR and was therefore considered a complication. It can be 
questioned whether this fatality is directly related to RALVR. Unfortunately, permission for autopsy was not obtained so this question will remain unanswered. This case represented the first mortality following rectopexy in our institution since 2004 after performing a total of more than 450 LVR and RALVR procedures. To our knowledge mortality after RALVR or after LVR has not been reported in literature before.

The learning curve for RALVR is very steep. In two learning curve studies the plateau phase for otherwise experiences laparoscopic surgeons was reached before 20 robot-assisted cases. After this curve is completed the results of RALVR are at least comparable to the results of LVR. The costs for RALVR however, are significantly higher compared to conventional LVR. This is mainly caused by more expensive disposables plus the costs for purchase and maintenance of the robot.[20] With the results of this current study these higher costs cannot yet be justified except for superior ergonomics, view and maneuverability for the surgeon. Therefore, prospective studies are needed with special regard to functional outcomes of LVR and RALVR, as these might be better after RALVR.

The main weakness of the current study is the retrospective design. As constipation/ODS and incontinence were evaluated in a standardized matter it was possible to determine the rate of incontinence and constipation/ODS before and after surgery. It was however not possible to assess the severity of these complaints since validated questionnaires were not routinely used. The preoperative questionnaire used in our department is for quick assessment of pelvic-organ-prolapse complaints but is not validated and does not quantify functional symptoms. Future studies should therefore use validated questionnaires (for example the Wexner scores) both pre- and postoperatively.[21;22]

In conclusion, RALVR is safe and effective for the treatment of internal RP, symptomatic rectocele and external RP. Due to better sight and maneuverability deep in the narrow pelvis functional results of RALVR might be superior to conventional LVR. This has to be addressed in large comparative trials. 


\section{REFERENCE LIST}

1 Altomare DF, Spazzafumo L, Rinaldi M, Dodi G, Ghiselli R, Piloni V: Set-up and statistical validation of a new scoring system for obstructed defaecation syndrome. Colorectal Dis 2008;10:84-88.

2 Hayden DM, Weiss EG: Fecal incontinence: etiology, evaluation, and treatment. Clin Colon Rectal Surg 2011;24:64-70.

3 Rao SS: Constipation: evaluation and treatment of colonic and anorectal motility disorders. Gastroenterol Clin North Am 2007;36:687-711, x.

4 Elneil S: Complex pelvic floor failure and associated problems. Best Pract Res Clin Gastroenterol 2009;23:555-573.

5 Braekken $\mathrm{IH}$, Majida M, Engh ME, Bo K: Can pelvic floor muscle training reverse pelvic organ prolapse and reduce prolapse symptoms? An assessor-blinded, randomized, controlled trial. Am J Obstet Gynecol 2010;203:170-177.

6 Hwang YH, Person B, Choi JS, Nam YS, Singh JJ, Weiss EG, Nogueras JJ, Wexner SD: Biofeedback therapy for rectal intussusception. Tech Coloproctol 2006;10:11-15.

7 D’hoore A, Penninckx F: Laparoscopic ventral recto(colpo)pexy for rectal prolapse: surgical technique and outcome for 109 patients. Surg Endosc 2006;20:1919-1923.

8 Formijne Jonkers HA, Draaisma WA, Wexner SD, Broeders IA, Bemelman WA, Lindsey I, Consten EC: Evaluation and surgical treatment of rectal prolapse: an international survey. Colorectal Dis 2013;15:115-119.

9 Formijne Jonkers HA, Poierrie N, Draaisma WA, Broeders IA, Consten EC: Laparoscopic ventral rectopexy for rectal prolapse and symptomatic rectocele: an analysis of 245 consecutive patients. Colorectal Dis 2013;15:695-699.

10 Wijffels N, Cunningham C, Lindsey I: Laparoscopic ventral rectopexy for obstructed defecation syndrome. Surg Endosc 2009;23:452.

11 Makela-Kaikkonen J, Rautio T, Klintrup K, Takala H, Vierimaa M, Ohtonen P, Makela J: Roboticassisted and laparoscopic ventral rectopexy in the treatment of rectal prolapse: a matched-pairs study of operative details and complications. Tech Coloproctol 2014;18:151-155.

12 Mantoo S, Podevin J, Regenet N, Rigaud J, Lehur PA, Meurette G: Is robotic-assisted ventral mesh rectopexy superior to laparoscopic ventral mesh rectopexy in the management of obstructed defaecation? Colorectal Dis 2013;15:e469-e475.

13 Paraiso MF, Barber MD, Muir TW, Walters MD: Rectocele repair: a randomized trial of three surgical techniques including graft augmentation. Am J Obstet Gynecol 2006;195:1762-1771.

14 Dindo D, Demartines N, Clavien PA: Classification of surgical complications: a new proposal with evaluation in a cohort of 6336 patients and results of a survey. Ann Surg 2004;240:205-213.

15 Boons P, Collinson R, Cunningham C, Lindsey I: Laparoscopic ventral rectopexy for external rectal prolapse improves constipation and avoids de novo constipation. Colorectal Dis 2010;12:526-532. 
16 Collinson R, Wijffels N, Cunningham C, Lindsey I: Laparoscopic ventral rectopexy for internal rectal prolapse: short-term functional results. Colorectal Dis 2010;12:97-104.

17 Perrenot C, Germain A, Scherrer ML, Ayav A, Brunaud L, Bresler L: Long-term outcomes of robotassisted laparoscopic rectopexy for rectal prolapse. Dis Colon Rectum 2013;56:909-914.

18 Boons P, Collinson R, Cunningham C, Lindsey I: Laparoscopic ventral rectopexy for external rectal prolapse improves constipation and avoids de novo constipation. Colorectal Dis 2010;12:526532.

19 Wijffels N, Cunningham C, Dixon A, Greenslade G, Lindsey I: Laparoscopic ventral rectopexy for external rectal prolapse is safe and effective in the elderly. Does this make perineal procedures obsolete? Colorectal Dis 2011;13:561-566.

20 Heemskerk J, de Hoog DE, van Gemert WG, Baeten CG, Greve JW, Bouvy ND: Robot-assisted vs. conventional laparoscopic rectopexy for rectal prolapse: a comparative study on costs and time. Dis Colon Rectum 2007:50:1825-1830.

21 Agachan F, Chen T, Pfeifer J, Reissman P, Wexner SD: A constipation scoring system to simplify evaluation and management of constipated patients. Dis Colon Rectum 1996;39:681-685.

22 Jorge JM, Wexner SD: Etiology and management of fecal incontinence. Dis Colon Rectum 1993;36:77-97. 


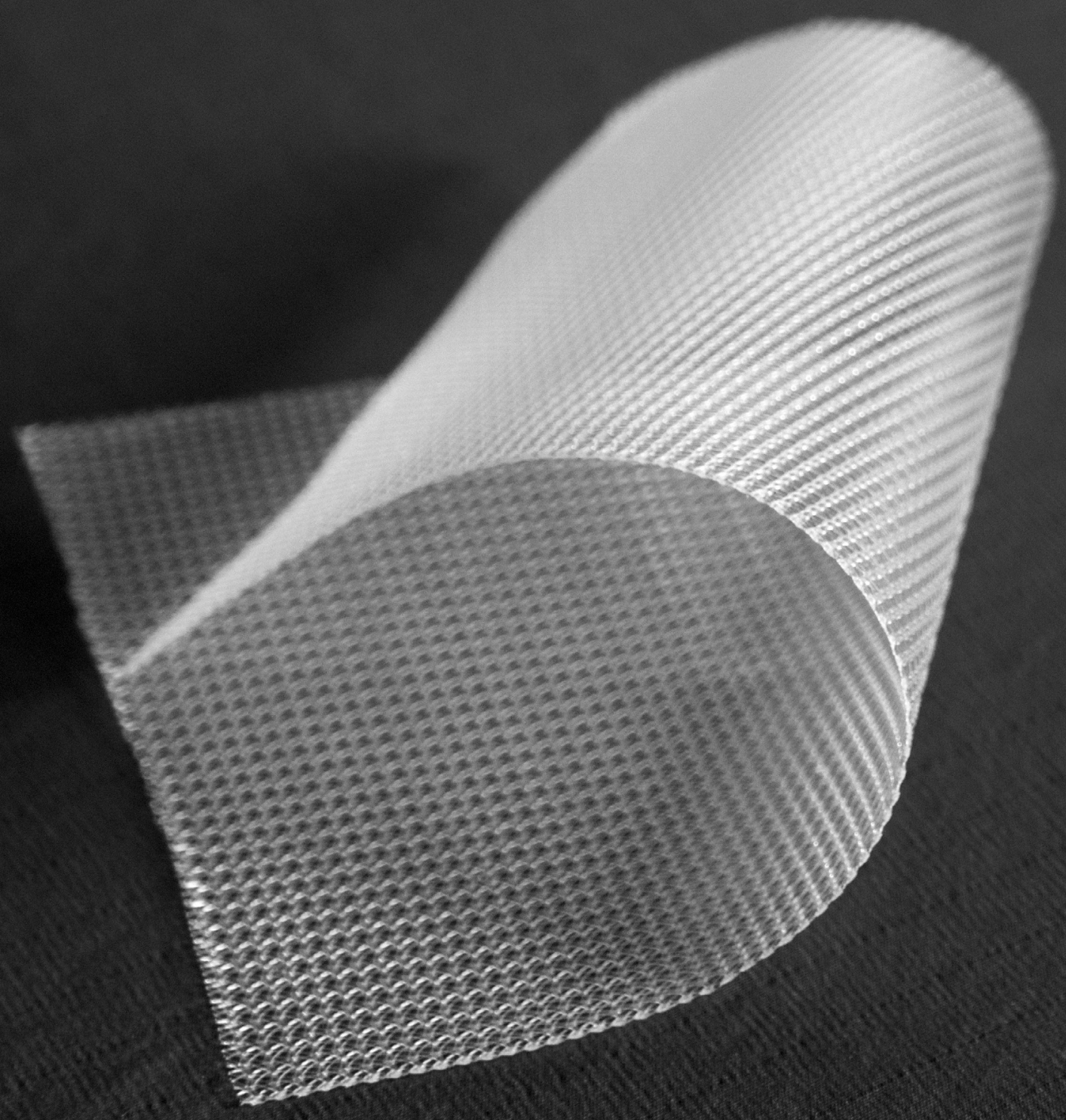




\section{Chapter 11}

General Discussion 
In this thesis, several aspects of the treatment of rectal prolapse (RP) were studied. Several research questions were formed as a guide during research. These questions are discussed in this chapter.

\section{WHAT IS CURRENT PRACTICE IN SURGERY FOR RP AND ARE THERE ANY DIFFERENCES BETWEEN SURGEONS AND COUNTRIES?}

The optimal treatment of RP has been discussed for decades. Numerous surgical techniques have been described in literature. Despite, or perhaps because of all these possibilities, no consensus has been reached and no guidelines have been created.

The lack of both consensus and guidelines is noticeably reflected in the results of the survey presented in chapter $\mathbf{2}$. No uniformity could be identified in the evaluation, treatment and follow-up of patients with either an internal or external RP. Consequently, major differences were found between treatment strategies in different international regions.

For healthy patients with an external RP, we found laparoscopic ventral rectopexy (LVR) to be the treatment of choice by the majority of surgeons throughout Europe. In the USA, laparoscopic resection rectopexy (LRR) is the primary treatment modality. For internal RP, both LVR and stapled-trans-anal-rectal-resection (STARR) are the preferred techniques in Europe after failure of conservative treatment. In the USA, LRR is used for healthy patients with persisting symptoms after conservative treatment of an internal RP.

In recent years, several studies showed the efficacy and safety of laparoscopic procedures in elderly patients (chapter 3)[1]. These findings have not led to a change in common practice as the majority of surgeons agreed on the treatment of frail and elderly patients with an external RP. Because of an old paradigm, perineal techniques were still preferred for this group of patients.

\section{WHAT ARE THE (FUNCTIONAL) RESULTS OF THE MOST POPULAR SURGICAL TECHNIQUES FOR THE TREATMENT OF RP?}

Surgery for RP and large symptomatic rectoceles is predominantly performed to decrease functional symptoms such as incontinence and constipation/ the obstructed defecation syndrome ODS. Several articles in this thesis focused on the functional results of conventional LVR and robot-assisted LVR (RALVR). In general, a significant postoperative reduction was found in symptoms of incontinence and constipation/ODS after both LVR and RALVR for internal RP, external RP and symptomatic rectoceles (chapters 3 and 10). The results of RALVR might be slightly better when compared to the results of conventional LVR: a total of $14 \%$ of patients had persisting symptoms of incontinence after LVR, compared to $9 \%$ after RALVR. For constipation/ODS this was $19 \%$ and $15 \%$, respectively. This might be due to better visibility and maneuverability deep in the pelvis during robot-assisted surgery, resulting in a better distal position of 
the mesh, closer to the pelvic floor muscles. Whether this difference is significant has to be addressed in large randomized future studies.

Constipation de-novo after ventral rectopexy is feared as an adverse effect of surgery as some surgeons believe that this procedure leads to 'kinking of the redundant sigmoid'. Analysis of the data presented in in this thesis revealed that the incidence of constipation de-novo was $1.5 \%$ in a combined total of 390 LVR/RALVR procedures. This is similar to other (smaller) series in literature.[2;3] Consequently, the theory of 'sigmoid kinking' can be rejected.

The results of LRR for the treatment of external RP were also investigated and directly compared to the results of LVR. This comparison can be found in chapter 5 . As well as LVR, LRR resulted in a significant reduction of incontinence and constipation/ODS. There were no significant differences between the two investigated techniques although the number of patients with persisting symptoms of incontinence was slightly higher after LVR $(P=0.06)$. Resection rectopexy however had a higher complication rate then did LVR.

Overall, both LRR and (RA)LVR do not always result in successful symptomatic outcome as this was not reported for all patients. The cause of incontinence and constipation is regularly multifactorial and the patients who did not improve after surgery may have had other underlying factors causing symptoms of incontinence and constipation, such as for example anal sphincter failure or colonic transit disorders. [4;5]Furthermore, the incidence of high grade hemorrhoids (grade III/IV mucosal prolapse) requiring surgical intervention was high during follow-up after (RA)LVR (15.4\%) (chapter 6). These hemorrhoids will influence the rate of incontinence and constipation during follow-up after (RA) LVR and seem to be associated with a higher recurrence rate of (complete) RP. Future research therefore needs to determine which patients do, and do not, benefit from rectopexy procedures and, subsequently, treatment algorithms for patients with RP and recurrences (either mucosal or full thickness) need to be established.

\section{WHAT IS THE INFLUENCE OF LVR ON THE SEXUAL FUNCTION OF FEMALE PATIENTS?}

We sought more evidence on the sexual function (SF) of females after LVR. These results are presented in the study in chapter 4. Prior to that study, one other study regarding this topic was published. It concluded that LVR improved sexual dysfunction.[6] These results were, however, established in a small cohort of patients ( $n=41$ ), without the use of validated questionnaires and could not be reproduced in our study. As described in chapter 4, the influence of LVR on sexual function seems limited in our large cohort of female patients. More than $40 \%$ of sexually active patients indicated that the LVR procedure did not effect their SF, while $16 \%$ of patients experienced an improved SF. Deterioration in SF during follow-up was experienced by $13 \%$ of patients. The remainder of pa- 
tients could not answer this question or were not sexually active. Additionally, the Pelvic Organ Prolapse-Urinary Incontinence Sexual Function Questionnaire (PISQ-12), a validated questionnaire to determine the SF of women with pelvic organ prolapse symptoms, was used to assess the SF of patients after LVR. The mean score was 34 points (maximum score for this questionnaire is 48 points). This is comparable to the results of other studies that investigated the SF of patients after various surgical procedures for the reduction of pelvic organ prolapse and slightly lower than the mean score of the general population.[7-11] In conclusion, the impact of LVR on the SF of patients seems limited.

\section{WHAT IS THE INCIDENCE OF MESH-RELATED COMPLICATIONS AFTER LVR?}

A safety warning published by the US Food and Drug Administration (FDA) concerning the use of synthetical meshes in pelvic organ prolapse surgery has led to negative publicity for this field of surgery. A high incidence of pain, mesh infections and mesh erosions through the vagina with subsequent dyspareunia were reported following mesh implantation for pelvic organ prolapse.[12] These reports concern meshes that were placed transvaginally, we sought to investigate whether LVR is accompanied with similar mesh related complications.

In chapter $\mathbf{3}$ of this thesis, the results of LVR are described. This cohort is the largest cohort of post-LVR patients described at the moment of publication. Only two cases of mesh infections $(0.8 \%)$ were found (after a mean follow-up of 30 months). For these, surgical removal of the mesh was performed in one case while the other patient could be treated with IV antibiotics. There were no cases of mesh erosion through the rectal or vaginal wall. Furthermore, LVR did not cause a significant deterioration in the SF for female patients, as can be appraised in chapter 4. It is however possible that there were cases of non-symptomatic erosions or that follow-up was not long enough to detect it.

Other large cohorts of patients treated with LVR show similar results concerning major mesh-related complications $(0.0$ - 1.9\%)[1;2;13;14] In a recent publication, results were published for a tertiary referral center for complications after LVR. In six years, 9 mesh erosions through the vaginal wall and 2 erosions through the rectal wall were treated as well as 3 cases of chronic pelvic pain. [15] As this was a referral center for both LVR and serious complications after LVR, the incidence of major complications could not be determined but seems low. Therefore, it can be concluded that LVR is a safe technique associated with a low incidence of (serious) mesh related complications. 


\section{WHICH OF THE POSSIBLE METHODS OF PROXIMAL MESH FIXATION DURING LVR IS PREFERABLE IN TERMS OF FIXATION STRENGTH?}

Several techniques can be used for proximal mesh fixation during LVR. These include non-resorbable stitches, tackers and a recently introduced screw.[13] This screw was designed for precise and strong fixation at the site of the sacral promontory. As these different techniques were not compared before, an ex-vivo experiment was conducted (chapter 5). The mean disruption force, which led to a break of the proximal mesh fixation, was 58 Newton with the use of three Protack tacks, 55 Newton using two stitches and 70 Newton when applying the new screw. The use of the new screw therefore led to a significantly stronger fixation compared to the use of stitches $(p=<0.05)$. No significant difference was determined between tacks and screw fixation. Therefore, it can be concluded that the new screw offers a strong fixation of the mesh at the site of the sacral promontory. Future research has to focus on the clinical implementation of this screw and the ease of use during LVR.

\section{WHAT IS THE CURRENT STATUS IN THE USAGE OF A ROBOTIC SURGERY SYSTEM IN THE FIELD OF RP SURGERY?}

Robot-assisted surgery seems to become the new operating standard for numerous procedures as these systems enhance visibility, maneuverability and dexterity during laparoscopic surgery. Because RP surgery is performed deep in the narrow pelvis, the use of a robot is considered a logical next step. Chapter $\mathbf{8}$ of this thesis describes the technique of RALVR. The preparations and surgical steps are described in detail. Furthermore, tips and pitfalls for this procedure are provided. Currently, only a few, small, studies have described the use of the Da-Vinci robot during RALVR.[16;17] These studies found satisfying results combined with a low rate of morbidity. The study presented in chapter 10 describes the largest cohort of patients treated with RALVR. In this study we found similar results, as discussed earlier in this general discussion. Hopefully, this will encourage the use of robot assisted techniques for rectopexy surgery. Future research is warranted to directly compare the results of LVR with those of RALVR.

\section{WHAT IS THE LEARNING CURVE FOR RALVR?}

In the study presented in chapter 9, the learning curve for RALVR of an otherwise experienced laparoscopic pelvic floor surgeon was investigated. We analyzed operating time and number of complications during the intial phase after implementation of the robot in our center. It was found that the learning curve was reached after 10 procedures for the console phase of the procedure and after 20 procedures for the total procedure. This is comparable to available literature, where the learning curve for the surgeon was reached within 20 procedures for RALVR and robot-assisted colorectal surgery in general.[16;17] Therefore, it can be concluded that the learning curve of RALVR is limited. 


\section{REFERENCE LIST}

1 Wijffels N, Cunningham C, Dixon A, Greenslade G, Lindsey I: Laparoscopic ventral rectopexy for external rectal prolapse is safe and effective in the elderly. Does this make perineal procedures obsolete? Colorectal Dis 2011;13:561-566.

2 Boons P, Collinson R, Cunningham C, Lindsey I: Laparoscopic ventral rectopexy for external rectal prolapse improves constipation and avoids de novo constipation. Colorectal Dis 2010;12:526-532.

3 Collinson R, Wijffels N, Cunningham C, Lindsey I: Laparoscopic ventral rectopexy for internal rectal prolapse: short-term functional results. Colorectal Dis 2010;12:97-104.

4 Hayden DM, Weiss EG: Fecal incontinence: etiology, evaluation, and treatment. Clin Colon Rectal Surg 2011;24:64-70.

5 Rao SS: Constipation: evaluation and treatment of colonic and anorectal motility disorders. Gastroenterol Clin North Am 2007;36:687-711, x.

6 Abet E, Lehur PA, Wong M, Rigaud J, Darnis E, Meurette G: Sexual function and laparoscopic ventral rectopexy for complex rectocoele. Colorectal Dis 2012;14:e721-e726.

7 Geller EJ, Parnell BA, Dunivan GC: Pelvic floor function before and after robotic sacrocolpopexy: one-year outcomes. J Minim Invasive Gynecol 2011;18:322-327.

8 Milani AL, Withagen MI, The HS, Nedelcu-van dW, I, Vierhout ME: Sexual function following trocarguided mesh or vaginal native tissue repair in recurrent prolapse: a randomized controlled trial. J Sex Med 2011;8:2944-2953.

9 Paraiso MF, Barber MD, Muir TW, Walters MD: Rectocele repair: a randomized trial of three surgical techniques including graft augmentation. Am J Obstet Gynecol 2006;195:1762-1771.

10 Rogers RG, Kammerer-Doak D, Villarreal A, Coates K, Qualls C: A new instrument to measure sexual function in women with urinary incontinence or pelvic organ prolapse. Am J Obstet Gynecol 2001;184:552-558.

11 Rogers RG, Coates KW, Kammerer-Doak D, Khalsa S, Qualls C: A short form of the Pelvic Organ Prolapse/Urinary Incontinence Sexual Questionnaire (PISQ-12). Int Urogynecol J Pelvic Floor Dysfunct 2003;14:164-168.

12 Rogers RG: To mesh or not to mesh: current debates in prolapse repair fueled by the U.S. Food and Drug Administration Safety Notification. Obstet Gynecol 2011;118:771-773.

13 D'hoore A, Penninckx F: Laparoscopic ventral recto(colpo)pexy for rectal prolapse: surgical technique and outcome for 109 patients. Surg Endosc 2006;20:1919-1923.

14 Tranchart H, Valverde A, Goasguen N, Gravie JF, Mosnier H: Conservative treatment of intrarectal mesh migration after ventral laparoscopic rectopexy for rectal prolapse. Int J Colorectal Dis 2013;28:1563-1566.

15 Badrek-Al Amoudi AH, Greenslade GL, Dixon AR: How to deal with complications after laparoscopic ventral mesh rectopexy: lessons learnt from a tertiary referral centre. Colorectal Dis 2013;15:707-712. 
16 Mantoo S, Podevin J, Regenet N, Rigaud J, Lehur PA, Meurette G: Is robotic-assisted ventral mesh rectopexy superior to laparoscopic ventral mesh rectopexy in the management of obstructed defaecation? Colorectal Dis 2013;15:e469-e475.

17 Perrenot C, Germain A, Scherrer ML, Ayav A, Brunaud L, Bresler L: Long-term outcomes of robotassisted laparoscopic rectopexy for rectal prolapse. Dis Colon Rectum 2013;56:909-914. 


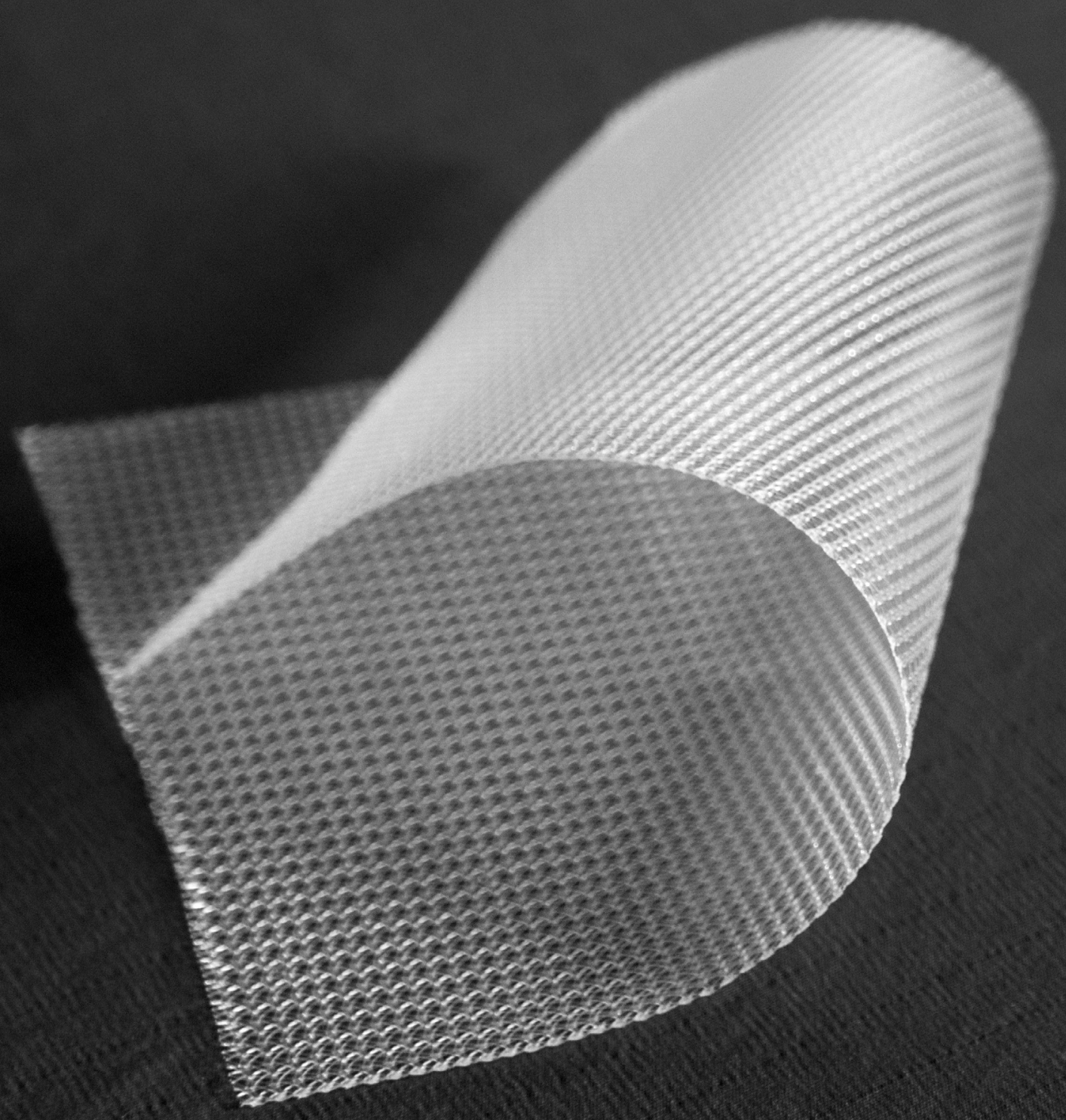




\section{Chapter 12}

Samenvatting in het Nederlands

Summary in Dutch 
Het rectum (de endeldarm) is het laatste deel van de dikke darm en daarmee tegelijk het laatste gedeelte van het maag-darm kanaal van de mens. De gemiddelde lengte van het rectum is tussen de 10 en 12 centimeter lang en de belangrijkste functie van het rectum is het tijdelijk vasthouden van ontlasting voordat deze het lichaam verlaat via de anus.

Verzakkingen van de organen in het kleine bekken is een frequent probleem. Met name bij vrouwen van 50 jaar of ouder die eenmaal of vaker zwanger zijn geweest komt dit vaak voor: ongeveer $50 \%$ krijgt dergelijke verzakkingsproblemen. De kans om hier ooit aan geopereerd te moeten worden is $11 \%$.

Behalve de blaas, baarmoeder en vagina is de endeldarm vaak betrokken in dit probleem. Een verzakking van het rectum kan zowel intern (als deze nog niet voorbij de kringspieren van de anus reikt) als extern zijn (waarbij de endeldarm daadwerkelijk door de anus naar buiten komt). Op het moment dat dergelijke verzakkingen van de endeldarm zich voor doen ("rectum prolaps") leidt dit met name tot functionele klachten van de endeldarm. Dit kan zowel incontinentie voor ontlasting zijn als klachten van obstipatie.

Voor externe rectum prolaps is de enige definitieve behandeling chirurgie. Voor interne prolaps wordt eerst een niet chirurgische behandeling geprobeerd (laxantia en bekkenbodem fysiotherapie). Als dit echter faalt dan is voor interne prolaps eveneens een chirurgische behandeling aangewezen.

De afgelopen 100 jaar zijn er tientallen chirurgische technieken beschreven om een rectum prolaps te opereren. Dit kan zowel met een buik operatie (abdominale benadering) of via een operatie vanaf het perineum (perneale benadering). Het voornaamste doel bij deze chirurgische behandelingen is het herstellen van de anatomie van het rectum en het verminderen van functionele klachten.

Alhoewel al tientallen chirurgische technieken zijn beschreven, zijn op het gebied van de behandeling van rectum prolaps nog vele vragen te beantwoorden. Enkele van deze vragen worden beantwoorden in dit proefschrift.

\section{WAT IS DE HUIDIGE CHIRURGISCHE BEHANDELING VAN RECTUM PROLAPS EN ZIJN ER VERSCHILLEN TUSSEN CHIRURGEN EN LANDEN?}

De optimale behandeling van rectum prolaps is al jaren een punt van discussie. Ondanks, of misschien juist wel door, al de verschillende chirurgische technieken die beschreven zijn, is er nog steeds geen consensus bereikt. Dit staat beschreven in hoofdstuk 2 van dit proefschrift.

In grote lijnen is Laparoscopische Ventrale Rectopexy (LVR) de meest populaire behandeling van externe rectum prolaps in Europa. Deze techniek is in Europa ook vaak gebruikt voor de behandeling van interne rectum prolaps na het falen 
van een conservatief behandel beleid. Perineale technieken (zoals STARR) zijn ook vaak gebruikt voor de behandeling van patiënten met een interne rectum prolaps. In de Verenigde Staten blijft echter - in tegenstelling tot Europa - Laparoscopische Resectie Rectopexy (LRR) het meest gebruikt voor deze groepen patiënten. De enige groep waar in Europa en de Verenigde Staten overeenstemming over bestaat de behandeling zijn oude ( $>80$ jaar) patiënten met een externe prolaps: deze worden zowel in Europa als in de Verenigde Staten bij voorkeur met perineale technieken behandeld, daar men geloofd dat dit minder complicaties met zich brengt.

\section{WAT ZIJN DE (FUNCTIONELE) RESULTATEN VAN DE MEEST GEBRUIKTE CHIRURGISCHE TECHNIEKEN VOOR DE BEHANDELING VAN RECTUM PROLAPS?}

Meerdere hoofdstukken in dit proefschrift gaan in op deze onderzoeksvraag. In hoofdstuk 3 worden de resultaten van LVR beschreven. Deze techniek wordt in toenemende mate uitgevoerd met een operatierobot (robot-geassisteerde LVR (RALVR)), waarvan de resultaten staan beschreven in hoofdstuk 10. Zowel voor LVR als voor RALVR werd een significante afname in klachten van incontinentie en obstipatie gezien na de operatie, vergeleken met de toestand vóór chirurgie. De resultaten van RALVR zijn wellicht zelfs nog beter dan die van LVR: in totaal had $14 \%$ van de patiënten persisterende incontinentie na LVR, vergeleken met $9 \%$ na RALVR. Voor obstipatie was dit $19 \%$ vs. $15 \%$. Dit verschil kan wellicht verklaard worden door een beter zicht op het operatiegebied tijdens robot geassisteerde chirurgie, gecombineerd met een verbeterde bewegingsvrijheid. Of dit verschil significant is zal moeten worden onderzocht tijdens grote, gerandomiseerde onderzoeken.

De resultaten van LRR voor de behandeling van externe rectum prolaps werden ook onderzocht en direct vergeleken met de resultaten van LVR. Deze studie staat beschreven in hoofdstuk 5. Net zoals bij LVR werd voor LRR een significante afname in incontinentie en obstipatie gevonden, vergeleken met de toestand vóór chirurgie. Tussen de twee technieken waren geen significante verschillen, al was het aantal patiënten met persisterende incontinentie na LVR hoger dan na LRR ( $p=0.06)$. Het aantal complicaties na LVR was echter weer significant lager dan na LRR.

\section{WAT IS DE INVLOED VAN LVR OP DE SEKSUELE FUNCTIE VAN VROUWELIJKE PATIËNTEN?}

Tijdens LVR wordt een mat gehecht tegen de voorwand van de rectum. Bij vrouwen betekent dit dat deze mat zich tevens tegen de achterwand van de vagina 
bevindt. Dit zou invloed kunnen hebben of de seksuele functie van vrouwen. Zo zou het kunnen dat de operatie zorgt voor een herstelde anatomie van de bekkenbodem en daarmee een beter seksueel functioneren. Aan de andere kant zou de mat ook pijnklachten kunnen veroorzaken. Hoofdstuk $\mathbf{4}$ van dit proefschrift is de eerste grote studie die focust op dit aspect van de operatie. Ongeveer $40 \%$ van de patiënten in dit onderzoek gaf aan dat de operatie geen invloed had gehad op de seksuele functie terwijl 16\% van de patiënten aangaf dat er een verbetering van de seksuele functie was opgetreden. Bij $13 \%$ van de patiënten vond er een verslechtering van de seksuele functie plaats.

In deze studie werd tevens een speciale, gevalideerde, vragenlijst voor het seksueel functioneren bij dergelijke bekkenbodem problematiek (PISQ-12 vragenlijst) voorgelegd aan de patiënten. De gemiddelde score bleek 34 punten (maximale score bij deze lijst is 48). Dit is slechts iets onder het gemiddelde van de algemene populatie (36 punten).

Concluderend lijkt daarmee de invloed van de operatie op de seksuele functie beperkt in deze groep patiënten.

\section{WAT IS DE INCIDENTIE VAN MAT GERELATEERDE COMPLICATIES NA LVR?}

De laatste jaren zijn chirurgische matten tegen verzakkingen van organen van de bekkenbodem, veelvuldig negatief in het nieuws geweest. Onder andere de Amerikaanse Food and Drug Administration (FDA) kwam in 2011 met een waarschuwing in verband met vermeende infecties van deze matten, erosies van de mat door organen heen en pijnklachten na dergelijke chirurgie. Dergelijke problemen treden met name op na het plaatsen van matjes door de vaginawand heen, hetgeen vaak wordt toegepast door gynaecologen bij verzakkingen van de vagina voor- en achterwand. De vraag is of dergelijke complicaties ook voorkomen na LVR.

In hoofdstuk $\mathbf{3}$ van dit proefschrift worden de resultaten van LVR beschreven in de grootste groep patiënten tot nu toe gepubliceerd. Slechts 2 gevallen van infectie van de mat werden gevonden in deze groep patiënten $(0.8 \%)$. Behandeling met intraveneuze antibiotica volstond in 1 van deze gevallen, bij de andere patiënt moest de mat chirurgisch verwijderd worden en werd een tijdelijk stoma aangelegd. Deze resultaten zijn in lijn met andere series van patiënten in de literatuur waarin een mat gerelateerd complicatie percentage van 0.0 tot $1.9 \%$ werd gevonden. Daarmee kan geconcludeerd worden dat LVR een veilige techniek is met een laag percentage mat gerelateerde complicaties. 


\section{WELKE METHODE VAN PROXIMALE MAT FIXATIE TIJDENS LVR IS HET STERKSTE?}

De mat welke wordt vastgehecht op de voorzijde van het rectum tijdens LVR moet ook op het promotorium worden vastgezet. Dit is een stuk bot aan de binnenkant van het bekken. Voor het vastzetten van de mat op het promotorium kan men verschillende technieken gebruiken: nietjes, tackers (een soort anker in de vorm van een kurkentrekker) of hechtingen. Recent werd tevens een speciaal schroefje hiervoor ontwikkeld en op de markt gebracht.

In hoofdstuk 5 van dit proefschrift werd de fixatie sterkte van de meest gebruikte methoden (hechtingen en tackers) vergeleken met de nieuwe schroef. Hiervoor werd de wervelkolom van een varken gebruikt in een ex-vivo diermodel. Na het vastzetten van de mat met deze drie verschillende methoden werd voor iedere methode de kracht gemeten waarmee de fixatie het begaf. Deze bleek 58 newton voor tackers, 55 newton voor hechtingen en 70 newton voor de nieuwe schroef. Geconcludeerd kan derhalve worden dat de nieuwe schroef - in vergelijking met de tackers en de hechtingen - sterke fixatie biedt van de mat aan het promotorium. Toekomstig onderzoek zal verder moeten focussen op de klinische implementatie van deze schroef.

\section{WAT IS DE HUIDIGE STATUS VAN HET GEBRUIK VAN EEN OPERATIE ROBOT GEDURENDE RECTUM PROLAPS CHIRURGIE?}

Robot geassisteerde chirurgie lijkt de nieuwe standaard te worden voor vele operaties. Dit komt door beter zicht en bewegelijkheid vergeleken met normale laparoscopische chirurgie. In hoofdstuk $\mathbf{8}$ wordt uitgebreid ingegaan op de techniek van RALVR. De voorbereidingen en stappen tijdens RALVR worden hierbij tot in detail beschreven. Verder worden tips en valkuilen beschreven.

Tot nu toe waren er slechts enkele kleine studies bekend die de resultaten van RALVR beschreven. In hoofdstuk $\mathbf{1 0}$ is het grootste cohort van patiënten na RALVR tot nu toe beschreven. Zoals eerder in deze samenvatting beschreven zijn de resultaten van RALVR op zijn minst vergelijkbaar met LVR. In de toekomst zullen LVR en RALVR direct met elkaar vergeleken moeten worden om eventuele verschillen aan te kunnen tonen.

\section{WAT IS DE ZOGENOEMDE "LEARNING CURVE" VOOR RALVR?}

Een chirurg die voor het eerst begint met het uitvoeren van complexere operaties zal door een fase heen moeten waarin hij of zij de operatie leert. Dit is de zogenoemde 'learning curve'. In hoofdstuk 9 werd de learning curve voor RALVR onderzocht. Het bleek dat deze voor de console fase (de tijd dat de chirurg daadwerkelijk achter de robot console zit) van de operatie na slechts 
10 procedures werd bereikt en na 20 procedures voor de totale operatie. Deze learning curve werd afgemeten aan de hand van de tijdsduur van de operatie en het aantal complicaties. Hieruit blijkt dat RALVR snel te leren is voor ervaren chirurgen op het gebied van bekkenbodem chirurgie. 


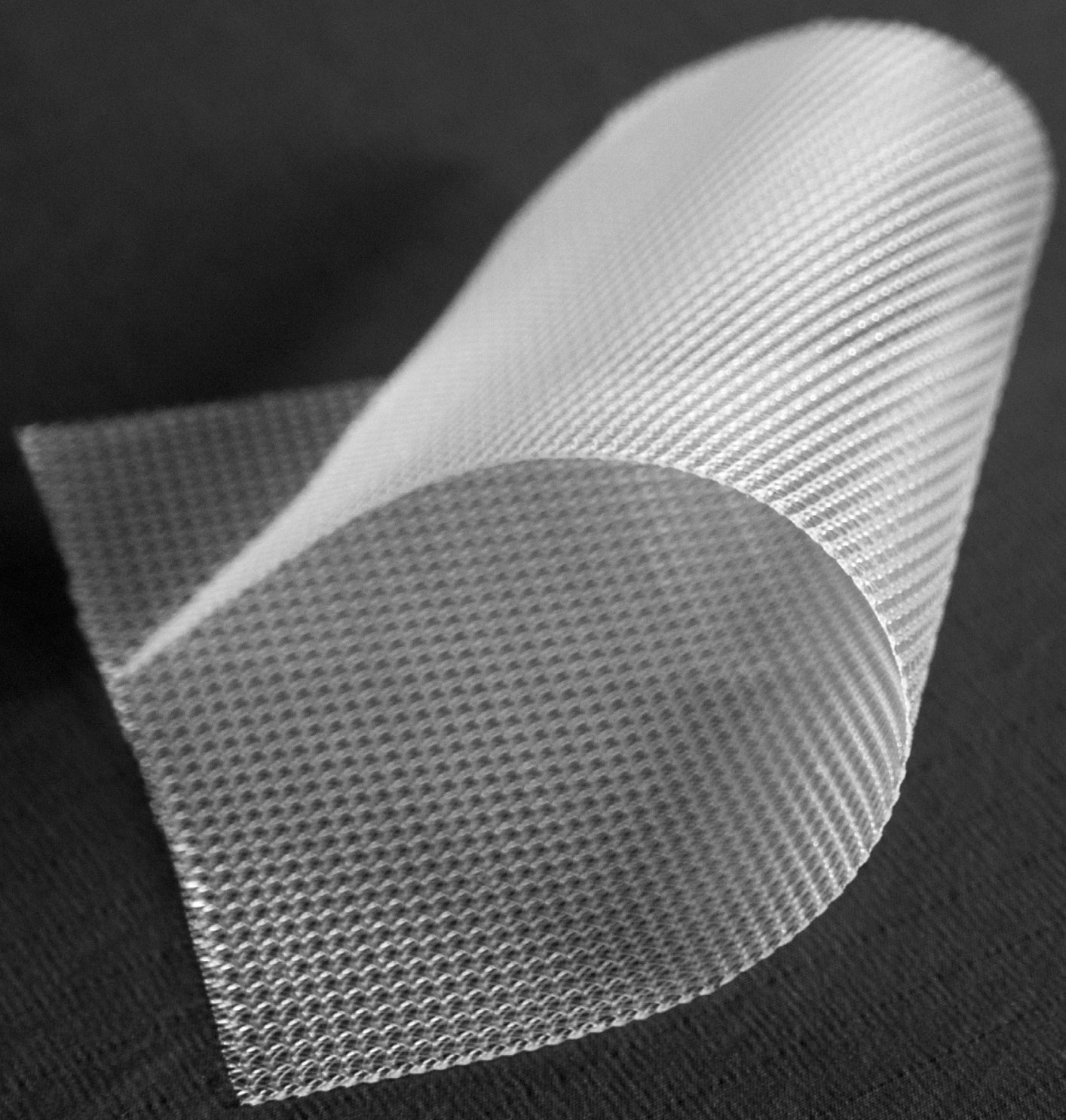


Review committee 
Prof. Dr. Ir. S. Stramigioli

Department of Robotics and Mechatronics

University of Twente

Enschede, The Netherlands

Prof. Dr. D.B.F. Saris

Department of Orthopedic Surgery

University Medical Center Utrecht

Utrecht, The Netherlands

and

MIRA institute

University of Twente,

Enschede, The Netherlands

Prof. Dr. A. D'Hoore

Department of Abdominal Surgery

University Hospital

Leuven, Belgium

Dr. M.F. Gerhards

Department of Surgery

Onze Lieve Vrouwe Gasthuis

Amsterdam, The Netherlands

Dr. R.K.J. Simmermacher

Department of Surgery

University Medical Center Utrecht

Utrecht, The Netherlands

Dr. S.J. van der Hagen

Department of Surgery

Refaja Hospital

Stadskanaal, The Netherlands 


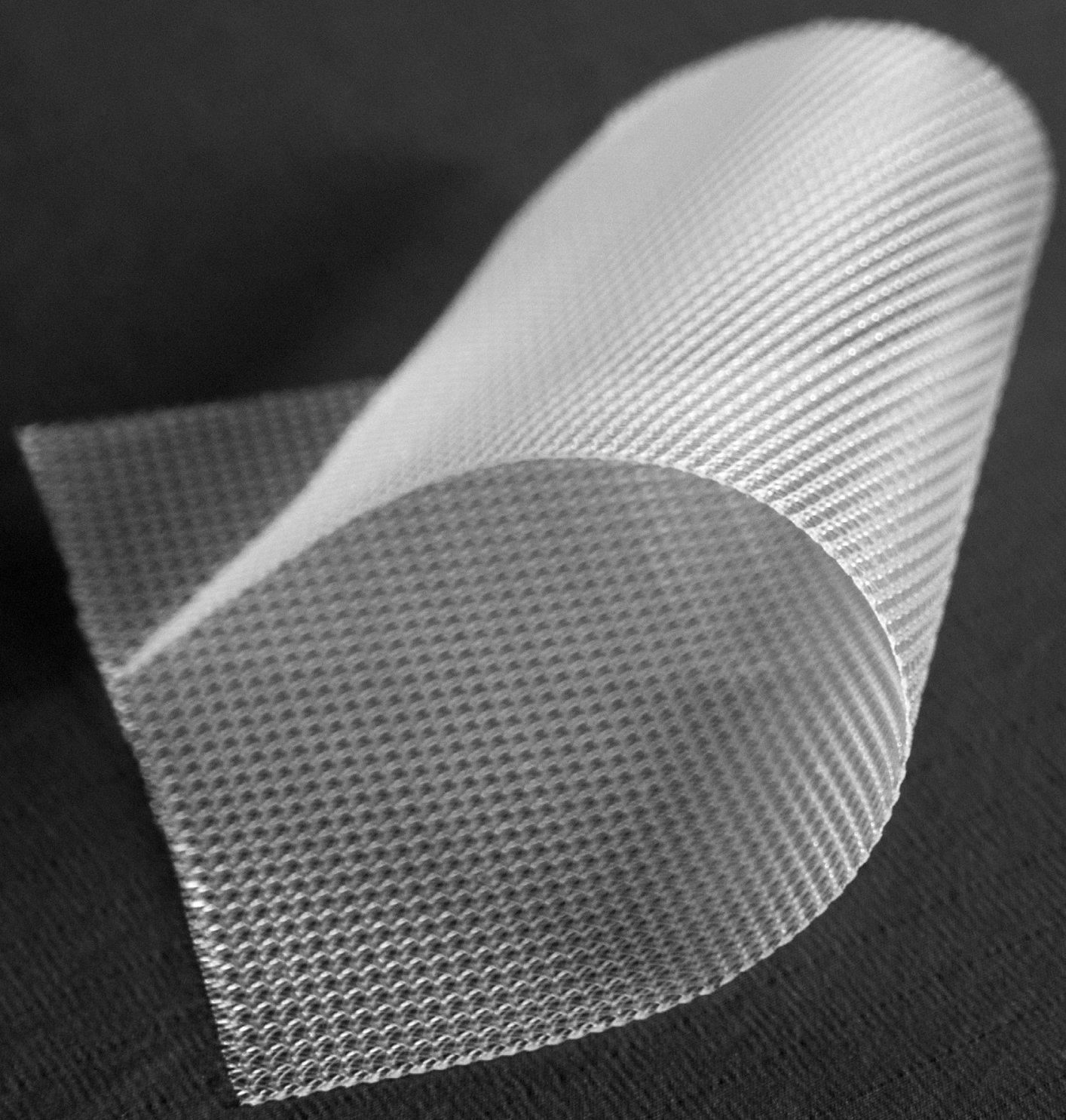


List of publications 
Early complications after stoma formation: a prospective cohort study in 100 patients with 1-year follow-up.

HA Formijne Jonkers, WA Draaisma WA, AM Roskott, AJ van Overbeeke, IAMJ Broeders and ECJ Consten

International Journal of Colorectal Disease. 2012 Aug;27(8):1095-9.

The optimal strategy for proximal mesh fixation during laparoscopic ventral rectopexy for rectal prolapse: an ex vivo study.

HA Formijne Jonkers, HJ van de Haar, WA Draaisma, BG Heggelman, ECJ Consten and IAMJ Broeders

Surgical Endoscopy. 2012 Aug;26(8):2208-12.

Evaluation and surgical treatment of rectal prolapse: an international survey. HA Formijne Jonkers, WA Draaisma, SD Wexner, IAMJ Broeders, WA Bemelman, I Lindsey and ECJ Consten Colorectal Disease. 2013 Jan;15(1):115-9.

Laparoscopic ventral rectopexy for rectal prolapse and symptomatic rectocele: an analysis of $\mathbf{2 4 5}$ consecutive patients.

HA Formijne Jonkers, N Poierrié, WA Draaisma, IAMJ Broeders and ECJ Consten

Colorectal Disease. 2013 Jun;15(6):695-9.

Impact of rectopexy on sexual function: a cohort analysis.

HA Formijne Jonkers, N Poierrié, WA Draaisma, IAMJ Broeders and ECJ

Consten

International Journal of Colorectal Disease. 2013 Nov;28(11):1579-82.

Laparoscopic resection rectopexy versus laparoscopic ventral rectopexy for complete rectal prolapse.

HA Formijne Jonkers, A Maya, WA Draaisma, WA Bemelman, IAMJ Broeders, ECJ Consten and SD Wexner

Techniques in Coloproctology. 2014.

Robotic-assisted Rectal Prolapse Surgery

HA Formijne Jonkers and IAMJ Broeders

Chapter in: Robotic surgery for the general surgeon. Nova Publishers, 2014. 


\section{Huidafwijkingen}

HA Formijne Jonkers and WMU van Grevenstein

Chapter in: Leidraad chirurgie, tweede editie. Bohn, Stafleu, Van Loghum

Publishers, 2014.

High-grade hemorrhoids requiring surgical treatment are common after laparoscopic ventral rectopexy

JJ van lersel, HA Formijne Jonkers, ECJ Consten, PM Verheijen, WA Draaisma and IAMJ Broeders

Submitted.

Learning curve for robot-assisted laparoscopic ventral rectopexy

HA Formijne Jonkers, N van der Stap, WA Draaisma, IAMJ Broeders and

ECJ Consten

Submitted.

Robot-Assisted Laparoscopic Ventral Rectopexy for rectal prolapse:

analysis of a large cohort of patients

HA Formijne Jonkers, JJ van lersel, WA Draaisma, PM Verheijen, IAM J

Broeders and ECJ Consten

Submitted. 


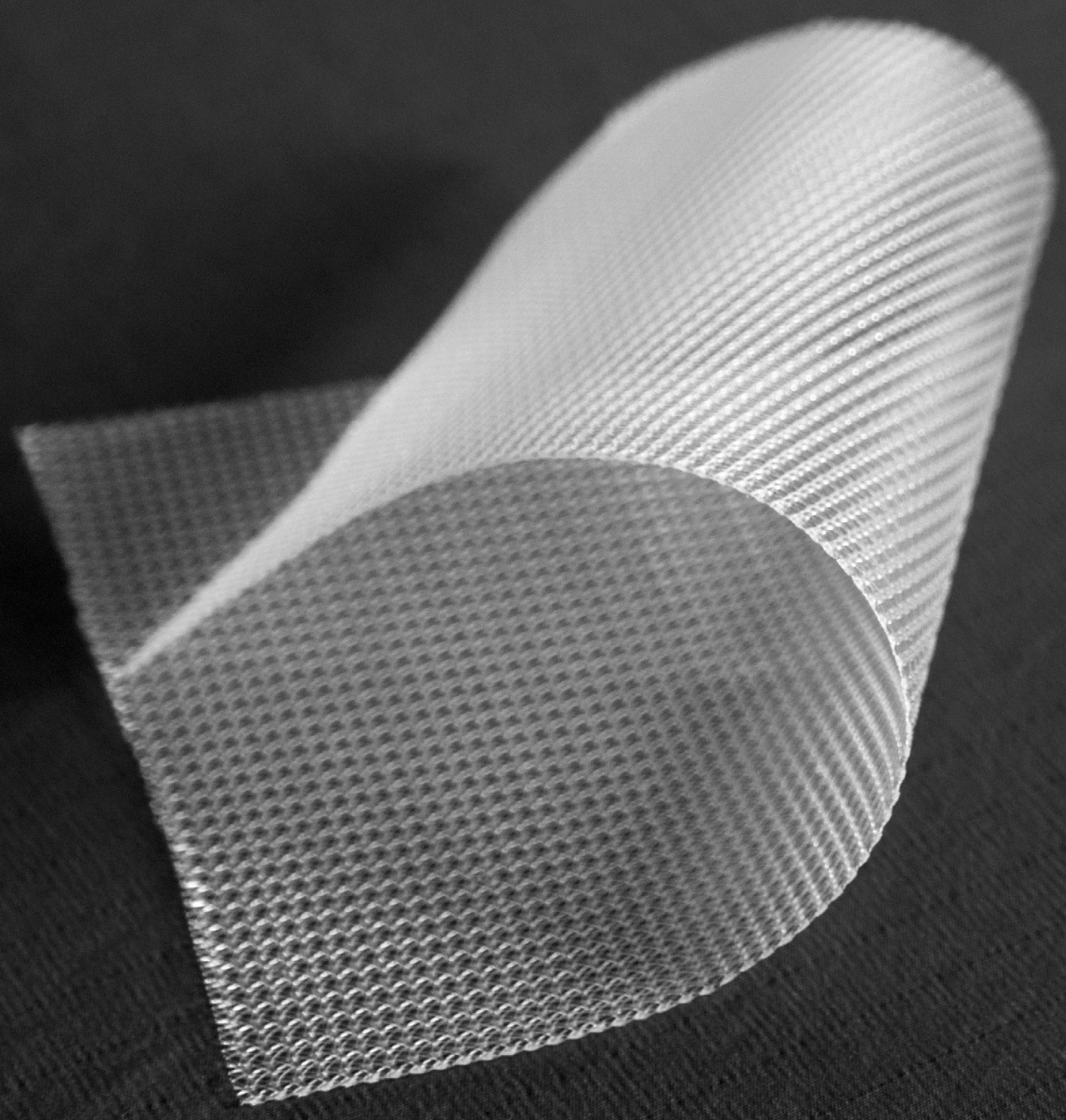


Dankwoord

Acknowledgements 
In het vijfde jaar van mijn studie Geneeskunde werd ik benaderd om onderzoek te verrichten bij de afdeling chirurgie van het Meander MC. Sindsdien heb ik ontzettend veel te danken aan de velen die mij gesteund hebben bij het tot stand komen van dit boek. De volgende personen verdienen een speciaal woord van dank.

Hooggeleerde promotor, Prof. Dr. I.A.M.J. Broeders. Beste Ivo. Inmiddels vier jaar geleden kreeg ik de kans om onder jou te promoveren. Nu ben ik ontzettend trots dat jij mijn promotor bent. Zonder jouw wetenschappelijke steun en inzichten was dit boek er nooit gekomen. Meer dan eens gaf je de goede richting aan voor het onderzoek en inmiddels ben ik overtuigd: "C'est la ton qui fait la musique".

Ook buiten de wetenschap bleek je een ware leermeester. Over de meest uitlopende zaken bracht je me de afgelopen jaren wijsheden bij die ik niet snel zal vergeten. Ik ben je enorm dankbaar voor dit alles en hoop nog veel van je te leren de komende jaren!

Hooggeleerde promotor, Prof. Dr. W.A. Bemelman. Beste Willem, vereerd was ik toen bleek dat jij graag samen met Ivo mijn promotor wilde zijn. Jij stond met jouw vele contacten in het buitenland, aan de basis van het 'internationale' karakter van mijn onderzoek. Dat was essentieel voor het succes van dit boek en de LaProS studie. Bedankt!

Hooggeachte co-promotor, Dr. E.C.J. Consten. Beste Esther. Jij was het die me tijdens mijn co-schap strikte voor onderzoek. Ik heb er geen moment spijt van gehad. Als echte "queen" van de onderzoeksgroep had je altijd weer ideeën voor verdere research, ritselde je financiering en arrangeerde ook nog even dat ik in Amerika onderzoek kon gaan doen. Dankzij jou is dit boekje er! Inmiddels is duidelijk dat jij mijn opleider zal zijn als ik weer terugkom in Amersfoort. Ik heb er zin in!

Hooggeachte co-promotor, Dr. W.A. Draaisma. Werner, vriend! Vanaf het begin van mijn onderzoek was jij diegene die me af en toe de noodzakelijke 'aanmoediging' gaf. Inmiddels ben je behalve co-promotor ook een goede vriend en ben ik je enorm dankbaar voor alle energie die je in me gestoken hebt. Ik kijk uit naar toekomstige reisjes! 
Prof. Dr. S.D. Wexner. You are one of the most famous surgeons throughout the world and after several years of collaborative research I totally understand why. Your enthusiasm and support were of great help during my research. Above all, I want to thank you for the opportunity to be one of your research fellows in Cleveland Clinic Florida. It has truly been one of the best times of my life.

De leden van de beoordelingscommissie, bestaande uit: Prof. Dr. Ir. S. Stramigioli, Prof. Dr. D.B.F. Saris, Prof. Dr. A. D'Hoore, Dr. M.F. Gerhards, Dr. R.K.J. Simmermacher en Dr. S.J. van der Hagen. Hartelijk dank voor uw bereidheid om dit proefschrift op wetenschappelijke waarde te beoordelen en plaats te nemen in de oppositie.

Mijn opleider in het UMC Utrecht: Dr. M.R. Vriens. Beste Menno, jouw eindeloze energie en toewijding maken jou een fantastische opleider. Elke dag geniet ik van mijn opleiding in het UMC. Dank voor je vertrouwen!

Chirurgen uit het UMC Utrecht. Ik wil jullie allemaal bedanken voor de fantastische tijd die ik op dit moment in het UMC heb. Utrecht: de allermooiste academie om opgeleid te worden!

Dr. A.J. van Overbeeke en alle chirurgen uit het Meander MC. Bij jullie heb ik de eerste echte stappen in de chirurgie gezet. Als beginnend assistent heb ik enorm veel van jullie geleerd en mede daardoor kwam ik in opleiding. Bedankt! $\mathrm{Na}$ twee academische jaren kom ik begin 2015 weer terug: ik kijk ernaar uit!

Intensivisten en Afdeling Intensive Care van Meander MC. Ik wil jullie bedanken voor de ontzettend leuke en leerzame tijd als ANIOS op de IC in 2012. Altijd begripvol als mijn interne DD niet veel langer dan 1 diagnose was (of nul) en ik eigenlijk aan dit onderzoek moest zitten...

Collega arts-assistenten Heelkunde uit het Meander MC en UMC Utrecht. Ik zeg vaak: "De helft van het plezier in je werk komt door je collega's". In jullie geval klopt dat zeker! Bedankt voor jullie steun en de vele gezellige momenten!

Antionio Maya. Antonio, buddy, I really enjoyed working together on the rectal prolapse studies in Florida. You got me an oral presentation during the "84th Congreso Argentino de Cirurgia" last year in Buenos Aires: it was awesome! Thanks for your hospitality and... "eres bienvenido en Holanda!!"

All other researchers of the department of Colorectal Surgery, Cleveland Clinic Florida: thank you for making my visit unforgettable! 
Dr. Bryan van de Wall. Brrrryyyyyaaaaaaan! Ooit begonnen we als jonge honden in de kelders van het oude Meander aan ons onderzoek. Vorig jaar stond ik achter jou. Vandaag ben jij mijn paranimf. Wie had gedacht dat het ooit zover zou komen? In de afgelopen jaren bleek dat we over veel dingen precies hetzelfde denken. Met weinig mensen heb ik dan ook zo hard gelachen als met jou tijdens de vele uren wetenschap, etentjes en mooie avonden. Bedankt voor je inspiratie en steun!

Philip Kremer. Flip! Ik vind het fantastisch dat jij vandaag mijn paranimf bent. We zij vanaf het begin in Utrecht goede jaarclubvrienden. Vele mooie verhalen beleefden we intussen op de kroeg, tijdens studeren in de bieb en op de vele ski-trips. Ik heb onwijs veel bewondering hoe je in no-time apotheker, dokter en neuroloog in opleiding werd en daarbij ook het nodige onderzoek deed! Maar genoeg serieus geluld: we moeten weer eens gaan skien! Catch you on the flip side!

Onderzoeksstudenten: Maartje Schouwenburg, Harm van de Haar en Nathalie Poierié. Beste onderzoekers, zonder jullie inzet was het een stuk lastiger geweest dit boekje te realiseren. Bedankt voor jullie werk! Ik hoop dat ik jullie wat heb kunnen bijbrengen over het opmerkelijke wereldje dat 'onderzoek doen' heet.

Mijn opvolger Jan van lersel. Jan, jongen! We kenden elkaar al lang voor je begon met mij werk uit handen te nemen. Inmiddels stalk jij met veel energie ouwe dametjes om de laatste 'functionele resultaten' uit ze te wringen. Je gaat als een dolle en ik weet zeker dat je binnen enkele jaren hier je boek zal verdedigen. Zet 'm op!

Natuurlijk wil ik al mijn andere vrienden en vriendinnen bedanken die mij de afgelopen jaren gesteund hebben. In het bijzonder:

Vrienden uit jaarclub Steiger. Na 11x een dies en 2 keer een lustrumreis zijn we inmiddels ouwe ballen. Ik vind het heel mooi dat we nog steeds als groep bij elkaar zijn na alle jaren. Van novitii in het Sticht tot waar we nu staan: wat hebben we samen veel meegemaakt! Teveel om op te noemen en zeker niet allemaal geschikt voor in dit boek. Vrienden voor het leven!

Pieter Visser. Maatje! Ik ben blij dat je weer in Utrecht bent komen wonen zodat we weer mooie avonturen kunnen gaan beleven! 
Tom van de Woestijne. Tom, we zijn vrienden met een uniek, bijna briljant, concept: mannenavond! Laten we deze traditie nog jaren voortzetten!

En natuurlijk last but certainly not least...

Henk en Gemma. Lieve papa en mama, zonder jullie had ik nooit zover kunnen komen! Door jullie steun, advies en liefde sta ik hier vandaag! Ik ben jullie eeuwig dankbaar!

Frans en Erik. Vanaf kleins af aan zijn we vrienden voor het leven. Inmiddels allemaal een ander leven en helaas zie ik jullie niet zo vaak als ik soms zou willen. Bedankt voor jullie steun. Jullie zijn fantastische broers!

Francijn. Liefste! Elke dag geniet ik van je! Jouw tomeloze energie, grappen en opmerkingen houden me scherp. Soms trapte je me letterlijk aan dit onderzoek en daarom ben jij een van de redenen dat dit boek er is! Ik hou van je en heb ontzettend zin in al onze toekomstige avonturen 


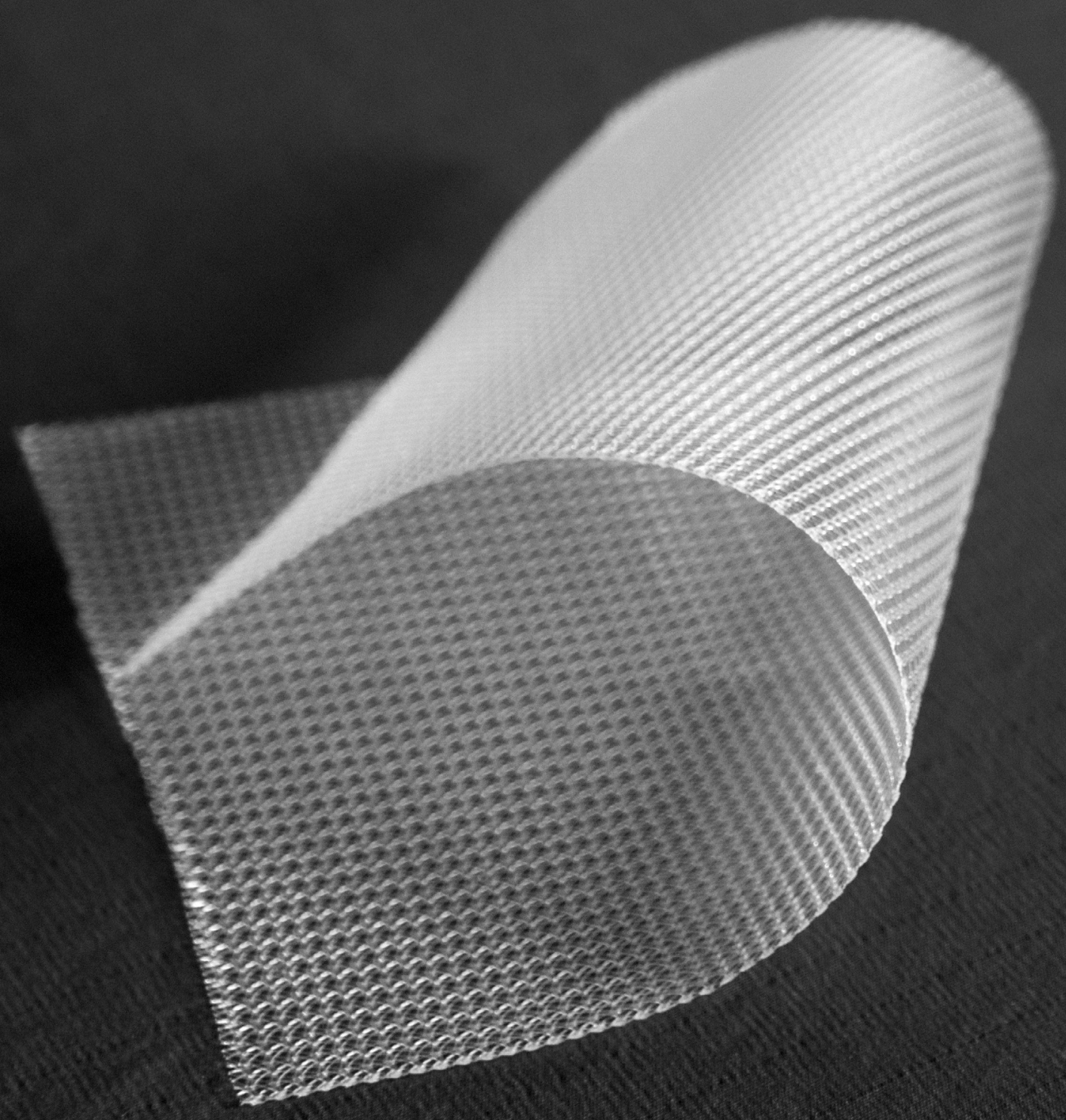


Curriculum vitae 
Henk Formijne Jonkers was born in Enschede, the Netherlands on the $11^{\text {th }}$ of June 1984. After graduating from the Staring College in Lochem (2002) he started to study Pharmaceutical Sciences (Utrecht University).

The next year he started Medical School at Utrecht University. As a student Henk developed a special interest in general surgery.

During his second rotation in general surgery he was asked by Dr. Werner Draaisma, Dr. Esther Consten and Prof. Dr. Ivo Broeders to join their surgical research group in the Meander Medical Center, Amersfoort. After finishing a prospective study on stoma complications, he initiated several studies in the field of rectal prolapse surgery.

After graduation from medical school in February 2010 Henk combined these research activities with working as a surgical resident and later as a resident on the Intensive Care Unit, both in the Meander Medical Center. From February 2012 till May 2012 Henk was given the opportunity to work at Cleveland Clinic Florida (FL, USA) as a research fellow under supervision of Prof. Dr. S.D. Wexner. During his period in the Meander Medical Center and the Cleveland Clinic he performed the work described in this thesis. The majority of these studies were also presented during various national and international conferences.

In January 2013 he started surgical training at the University Medical Center Utrecht under supervision of Dr. Menno Vriens. From January 2015 on, he will continue his residency at the Meander Medical Center under supervision of Dr. Esther Consten. 
Curriculum Vitae 